\title{
COULOMB GAS ENSEMBLES AND LAPLACIAN GROWTH
}

\author{
HÅKAN HEDENMALM AND NIKOLAI MAKAROV
}

\begin{abstract}
Авstract. We consider weight functions $Q: \mathbb{C} \rightarrow \mathbb{R}$ that are locally in a suitable Sobolev space, and impose a logarithmic growth condition from below. We use $Q$ as a confining potential in the model of one-component plasma (2-dimensional Coulomb gas), and study the configuration of the electron cloud as the number $n$ of electrons tends to infinity, while the confining potential is rescaled: we use $m Q$ in place of $Q$ and let $m$ tend to infinity as well. We show that if $m, n$ tend to infinity in a proportional fashion, with $n / m \rightarrow t$, where $0<t<+\infty$ is fixed, then the electrons accumulate on a compact set $S_{t}$, which we call the droplet. The set $S_{t}$ can be obtained as the coincidence set of an obstacle problem, if we remove a small set (the shallow points). Moreover, on the droplet $S_{t}$, the density of electrons is asymptotically $\Delta Q$. The growth of the droplets $S_{t}$ as $t$ increases is known as Laplacian growth. It is well-known that Laplacian growth is unstable. To analyze this feature, we introduce the notion of a local droplet, which involves removing part of the obstacle away from the set $S_{t}$. The local droplets are no longer uniquely determined by the time parameter $t$, but at least they may be partially ordered. We show that the growth of the local droplets may be terminated in a maximal local droplet, or by the droplets' growing to infinity in some direction ("fingering").
\end{abstract}

\section{Overview}

1.1. Outline of the paper. In Sections 2 and 3 , we study the one-component plasma (Coulomb gas ensemble) in two dimensions, and find the quasi-classical limit as the number $n$ of electrons tends to infinity while the confining potential is rescaled: $m Q$ replaces $Q$, where $m$ tends to infinity, so that $n / m \rightarrow t$. This was obtained previously by Johansson [17] in the one-dimensional context. It turns out that Johansson's proof carries through with only minor modifications also in the two-dimensional case, as was explained earlier in our arXiv preprint [13]. Here, we make an effort to obtain the result under minimal smoothness and growth assumptions on the potential $Q$.

In Section 4, we connect the equilibrium measure with an obstacle problem, and show how to apply the Kinderlehrer-Stampacchia-Caffarelli theory to obtain a priori smoothness of the solutions to the obstacle problem. We also show that the density of the equilibrium measure is given by $\Delta Q$ on the droplet, which permits us to reduce the complexity of the equilibrium measure to the study of its support (the droplet). Here, $\Delta:=\partial \bar{\partial}$ is a quarter of the usual Laplacian. The droplet is shown to equal the coincidence set for the associated obstacle problem, if we remove the so-called shallow points. For smooth strictly convex $Q$, the topology of the droplets is shown to be simple.

In Section 5, we introduce the notion of local droplets, which are obtained when we pass from the potential $Q$ to its localization $Q_{\Sigma}$ for subsets $\Sigma \subset \mathbb{C}$ (cf., e.g. [8]). The local droplets are partially ordered, and in Section 6 , we study maximal domination chains of local droplets. The maximal domination chains either end in a maximal local droplet, or grow to infinity. The

The first author is supported by the Göran Gustafsson Foundation (KVA) and Vetenskapsrådet (VR). The second author is supported by NSF Grant No. 0201893. 
local droplets appear to be natural from the point of view of physics (see, e.g., [21]). They are also natural from the mathematical point of view: the description of all possible local droplets is exactly the inverse problem of potential theory.

One purpose with the material on domination chains of droplets in Section 6 is to provide a natural setting to analyze Laplacian growth (i.e., the Hele-Shaw equation), which is known to be unstable in the forward time direction. This is explained in Section 7 . The domination chains of droplets are interesting in part because of their integrability nature, especially in the case of potentials $Q$ with $\Delta Q=$ constant $>0$ near the local droplet (such $Q$ will be called constant strength potentials). This will be the topic of a forthcoming paper, where we will discuss the algebraic-geometric nature of maximal local droplets for constant strength potentials.

1.2. Comments on the exposition. While a few of the results covered in this paper are essentially understood, we believe the reader will appreciate a rather self-contained and easily accessible exposition. As for the treatment of Johansson's theorems in Section 3 , the extension to the twodimensional setting requires some care about details, and as far as we know, no general proof has been available so far beyond the arXiv preprint [13], where an excessive regularity condition was made to simplify the presentation (here, we remove that condition by modifying the smoothing argument of Johansson's paper [17]; see Subsection 3.2).

The connection between equilibrium measures and obstacle problems is known (see, e.g., [20]). However, it is perhaps less well known that the Kinderlehrer-Stampacchia-Caffarelli theory (see [18]; cf. also [16], where the same technique was used) allows us to develop an understanding of the equilibrium measures in terms of their supports, the droplets. This contrasts with the onedimensional theory, where a lot of the difficulty is to determine the density of the equilibrium measure. As for the treatment of the Hele-Shaw equation, our approach based on equilibrium measures and obstacle problems allows us to develop the theory with low regularity. The standard approach to Hele-Shaw flow theory is to use (partial) balayage and variational inequalities, see, e.g., [12]. We prefer the obstacle problem approach because it is more intuitive and geometrically appealing.

1.3. Acknowledgements. We thank Kurt Johansson for helpful comments in connection with the previous arXiv preprint [13], and Serguei Shimorin for help with the proofreading.

\section{Quasi-classical limit of Coulomb gas ensembles}

2.1. One-component plasma (OCP). In the 2-dimensional Coulomb gas model (or rather OCP, the one-component plasma model), we have $n$ electrons located at points $\left\{z_{j}\right\}_{j=1}^{n}$ in the complex plane, influenced by an external field. The potential of interaction is

$$
\log \frac{1}{\left|z_{j}-z_{k}\right|^{2}}, \quad j \neq k, \quad j, k \in\{1, \ldots, n\},
$$

while the external field potential is denoted by $V(z)$. The function

$$
V: \mathbb{C} \rightarrow \mathbb{R} \cup\{+\infty\}
$$

is lower semi-continuous and sufficiently large to keep the electrons at finite distances. We shall supply the precise condition shortly. The combined potential energy resulting from particle interaction and the external potential is the function $\mathcal{E}_{V}: \mathbb{C}^{n} \rightarrow \mathbb{R} \cup\{+\infty\}$ given by

$$
\mathcal{E}_{V}(z)=\frac{1}{2} \sum_{j, k: j \neq k} \log \frac{1}{\left|z_{j}-z_{k}\right|^{2}}+\sum_{j} V\left(z_{j}\right), \quad z=\left(z_{1}, \ldots, z_{n}\right) \in \mathbb{C}^{n},
$$

where the summation indices $j, k$ are assumed confined to the set $\{1, \ldots, n\}$. In any reasonable gas dynamics model, the low energy states are supposed to be more likely than the high energy 
states. For a positive constant $\beta$, let $Z_{n}=Z_{n, \beta, V}$ denote the constant

$$
Z_{n}=\int_{\mathbb{C}^{n}} \mathrm{e}^{-\frac{\beta}{2} \mathcal{E}_{V}} \mathrm{dvol}_{2 n}
$$

where $\operatorname{vol}_{2 n}$ denotes the standard volume measure in $\mathbb{C}^{n} \cong \mathbb{R}^{2 n}$. We suppose that $0<Z_{n}<+\infty$, which means that the potential $V$ imposes a weak localization restraint on the plasma cloud. The corresponding Gibbs model then gives the joint density of states

$$
\frac{1}{Z_{n}} \mathrm{e}^{-\frac{\beta}{2} \mathcal{E}_{V}(z)} \text {. }
$$

where $\beta$ has the interpretation as the inverse temperature. In terms of the usual van der Monde expression

$$
\Delta(z)=\prod_{j, k: j<k}\left(z_{k}-z_{j}\right)
$$

we may write

$$
Z_{n}=\int_{\mathbb{C}^{n}}|\Delta(z)|^{\beta} \mathrm{e}^{-\frac{\beta}{2} \sum_{j} V\left(z_{j}\right)} \operatorname{dvol}_{2 n}(z) .
$$

We thus introduce a probability point process

$$
\Pi_{n} \equiv \Pi_{n, \beta, V} \in \operatorname{prob}\left(\mathbb{C}^{n}\right)
$$

$\left(\operatorname{prob}\left(\mathbb{C}^{n}\right)\right.$ is the convex set of all Borel probability measures on $\left.\mathbb{C}^{n}\right)$ by setting

$$
\mathrm{d} \Pi_{n}(z)=\frac{\mathrm{e}^{-\frac{\beta}{2} \varepsilon_{V}(z)}}{Z_{n}} \operatorname{dvol}_{2 n}(z)=\frac{|\Delta(z)|^{\beta}}{Z_{n}} \mathrm{e}^{-\frac{\beta}{2} \sum_{j} V\left(z_{j}\right)} \operatorname{dvol}_{2 n}(z), \quad z \in \mathbb{C}^{n} .
$$

2.2. Marginal measures. For integers $k=1, \ldots, n$, we define the marginal probability measure $\Pi_{n}^{(k)} \in \operatorname{prob}\left(\mathbb{C}^{k}\right)$ by setting

$$
\Pi_{n}^{(k)}(e)=\Pi_{n}\left(e \times \mathbb{C}^{n-k}\right),
$$

for Borel measurable subsets $e \subset \mathbb{C}^{k}$; in particular, $\Pi_{n}^{(n)}=\Pi_{n}$. The associated measures

$$
\Gamma_{n}^{(k)}=\frac{n !}{(n-k) !} \Pi_{n}^{(k)}
$$

are known as intensity (or correlation) measures. For $k=n$, we have $\Gamma_{n}^{(n)}=n ! \Pi_{n}$, which is why we simplify the notation and write $\Gamma_{n}=\Gamma_{n}^{(n)}$. On the other hand, for $k=1$, we have $(\mathbb{E}$ is the expectation operation)

$$
\Gamma_{n}^{(1)}(e)=\mathbb{E}\left[\#\left\{j: z_{j} \in e\right\}\right]
$$

where it is tacitly assumed that $j$ is confined to the set $\{1, \ldots, n\}$, and \# denotes counting measure. In more explicit form, we have, for $n=2$ and $k=1$,

$$
\mathrm{d} \Gamma_{2}^{(1)}(\zeta)=\frac{2 \int_{\mathbb{C}}|\zeta-\xi|^{\beta} \mathrm{d} \mu(\xi)}{\int_{\mathbb{C}^{2}}|\xi-\eta|^{\beta} \mathrm{d} \mu(\xi) \mathrm{d} \mu(\eta)} \mathrm{d} \mu(\zeta), \quad \zeta \in \mathbb{C},
$$

where $\mathrm{d} \mu(\xi)=\mathrm{e}^{-\frac{\beta}{2} V(\xi)} \mathrm{dvol}_{2}(\xi)$. More generally, for $1 \leq k \leq n$ and a Borel subset $e \subset \mathbb{C}^{k}$, we have

$$
\Gamma_{n}^{(k)}(e)=\mathbb{E}\left[\#\left\{\left(j_{1}, \ldots, j_{k}\right) \in \operatorname{perm}(k, n):\left(z_{j_{1}}, \ldots, z_{j_{k}}\right) \in e\right\}\right],
$$

where perm $(k, n)$ stands for the collection of all permutations of length $k$ of the set $\{1, \ldots, n\}$.

Remark 2.1. In the above definition of the probability measure $\Pi_{n}$, we realize that

$$
\mathrm{e}^{-\frac{\beta}{2} \sum_{j} V\left(z_{j}\right)} \mathrm{dvol}_{2 n}(z)=\mathrm{d} \mu\left(z_{1}\right) \cdots \mathrm{d} \mu\left(z_{n}\right), \quad z=\left(z_{1}, \ldots, z_{n}\right),
$$

where

$$
\mathrm{d} \mu(\xi)=\mathrm{e}^{-\frac{\beta}{2} V(\xi)} \mathrm{dvol}_{2}(\xi), \quad \xi \in \mathbb{C} .
$$


Most of the above discussion does not depend on this particular structure of the measure $\mu$, and we are free to consider more general measures. For instance, this allows us to include the one-dimensional theory in the model.

2.3. The random normal matrix model. If $\beta=2$, then the probability measure

$$
\mathrm{d} \Pi_{n}(z)=\frac{|\Delta(z)|^{2}}{Z_{n}} e^{-\sum_{j} V\left(z_{j}\right)} \operatorname{dvol}_{2 n}(z)
$$

with normalization constant

$$
Z_{n}=\int_{\mathbb{C}^{n}}|\Delta(z)|^{2} e^{-\sum_{j} V\left(z_{j}\right)} \operatorname{dvol}_{2 n}(z)
$$

describes the distribution of the eigenvalues of $n \times n$ Random Normal Matrices (RNM) with joint probability measure proportional to

$$
e^{-\operatorname{tr} V(M)} \mathrm{d} M,
$$

where " $\operatorname{tr}$ " is the trace, and $\mathrm{d} M$ stands for the natural "Haar-type" measure on the submanifold of all complex-valued $n \times n$ matrices $M$ with $M^{*} M=M M^{*}$ (the normal matrices). In this case the point process is determinantal:

$$
\mathrm{d} \Gamma_{n}^{(k)}(z)=\operatorname{det}\left[K_{n}\left(z_{i}, z_{j}\right)\right]_{i, j=1}^{k} e^{-\sum_{j} V\left(z_{j}\right)} \operatorname{dvol}_{2 k}(z),
$$

where $K_{n}$ is the reproducing kernel in the polynomial Bargmann-Fock space

$$
\operatorname{Pol}_{n}=\operatorname{span}\left\{1, z, \ldots, z^{n-1}\right\} \subset L^{2}\left(\mathbb{C}, \mathrm{e}^{-V}\right) .
$$

We thus consider $\operatorname{Pol}_{n}$ as a finite-dimensional linear subspace of $L^{2}\left(\mathbb{C}, e^{-V}\right)$ (linearity is always with respect to the field $\mathbb{C}$ ), and the Gram-Schmidt procedure supplies, for $j=0, \ldots, n-1$, polynomials $p_{j}$ of degree $j$ and norm 1 such that $p_{j} \perp p_{k}$ for $j \neq k$. In terms of these orthogonal polynomials, we have

$$
K_{n}(z, w)=\sum_{0}^{n-1} p_{j}(z) \bar{p}_{j}(w) .
$$

The algebraic mechanism behind the formula for the correlation measure $\Gamma_{n}^{(m)}$ is well understood. See, for instance, Mehta's book [19].

2.4. Aggregation of quantum droplets. For reasons that will become clearer later on, we shall regard the point process $\Gamma_{n}=n ! \Pi_{n}$ (or, equivalently, $\Pi_{n}$ ) as a quantum droplet. We are interested in the transition $\Gamma_{n} \rightarrow \Gamma_{n+1}$, which corresponds to adding one more electron to the droplet. A direct comparison of the processes $\Gamma_{n}$ and $\Gamma_{n+1}$ is not possible, and we are led to consider marginal intensities. The following lemma for $\beta=2$ has the interpretation that if we add an electron, the expected number of $k$-tuples of electrons increases everywhere in $\mathbb{C}^{k}$.

Lemma 2.2. If $\beta=2$, then

$$
\forall k, \quad \Gamma_{n}^{(k)} \leq \Gamma_{n+1}^{(k)}
$$

Proof. In view of (2.3), we have

$$
\left[K_{n+1}\left(z_{i}, z_{j}\right)\right]_{i, j=1}^{k}=\left[K_{n}\left(z_{i}, z_{j}\right)\right]_{i, j=1}^{k}+\left[p_{n}\left(z_{i}\right) \bar{p}_{n}\left(z_{j}\right)\right]_{i, j=1}^{k},
$$

where all matrices involved are positive (semi)definite (the rightmost matrix has rank 1 ). As we compare with (2.2), we realize that the desired assertion

$$
\operatorname{det}\left[K_{n}\left(z_{i}, z_{j}\right)\right]_{i, j=1}^{k} \leq \operatorname{det}\left[K_{n+1}\left(z_{i}, z_{j}\right)\right]_{i, j=1}^{k}
$$


is an immediate consequence of the minimax principle (see, e. g., the books of Dunford, Schwarz [7] and Gohberg, Krein [11]).

Remark 2.3. This "aggregation" property might well be true for all $\beta \leq 2$ but it certainly fails for $\beta>2$. We consider the illuminating special case $\Gamma_{1}^{(1)} \leq \Gamma_{2}^{(1)}$, which in the notation of (2.1) asserts that

$$
\int_{\mathbb{C}^{2}}|\xi-\eta|^{\beta} \mathrm{d} \mu(\xi) \mathrm{d} \mu(\eta) \leq 2 \mu(\mathbb{C}) \int_{\mathbb{C}}|\zeta-\xi|^{\beta} \mathrm{d} \mu(\xi), \quad \zeta \in \mathbb{C} .
$$

The measure $\mathrm{d} \mu(\xi)=\mathrm{e}^{-\frac{\beta}{2} V(\xi)} \mathrm{dvol}_{2}(\xi)$ can essentially be replaced by an fairly arbitrary positive Borel measure (with finite moments). As we plug in the choice $\mathrm{d} \mu=\mathrm{d} \delta_{0}+\mathrm{d} \delta_{1}$, we see that (2.4) is equivalent to

$$
|\zeta|^{\beta}+|\zeta-1|^{\beta} \geq \frac{1}{2}, \quad \zeta \in \mathbb{C} .
$$

With $\zeta=\frac{1}{2}$ this gives $\beta \leq 2$. In fact, it is possible to show that the inequality $\Gamma_{1}^{(1)} \leq \Gamma_{2}^{(1)}$ holds generally for $0<\beta \leq 2$. We outline the argument. It suffices to consider $z=0$ in (2.4), and to show that

$$
\int_{\mathbb{C}^{2}}\left\{|\xi|^{\beta}+|\eta|^{\beta}-|\xi-\eta|^{\beta}\right\} \mathrm{d} \mu(\xi) \mathrm{d} \mu(\eta) \geq 0
$$

for all positive measures $\mu$ with finite moments. For $0<\beta \leq 1$ the $L^{\beta}$ triangle inequality shows that the integrand on the left hand side is positive point-wise, and the assertion is immediate. We turn to the remaining case $1<\beta<2$. One first establishes with the methods of Calculus that

$$
(1+t+2 x)^{\beta / 2} \leq 1+t^{\beta / 2}+\beta x, \quad-\sqrt{t} \leq x \leq \sqrt{t}, 0<t<+\infty,
$$

which in complex form becomes

$$
|1+\tau|^{\beta} \leq 1+|\tau|^{\beta}+\beta \operatorname{Re} \tau, \quad \tau \in \mathbb{D},
$$

where $\mathbb{D}$ denotes the open unit disk in $\mathbb{C}$. By homogenization, this inequality leads to

$$
|\xi-\eta|^{\beta} \leq|\xi|^{\beta}+|\eta|^{\beta}-\beta \min \left\{|\xi|^{\beta-2},|\eta|^{\beta-2}\right\} \operatorname{Re}(\bar{\eta} \xi), \quad \xi, \eta \in \mathbb{C},
$$

so that

$$
|\xi|^{\beta}+|\eta|^{\beta}-|\xi-\eta|^{\beta} \geq \beta \min \left\{|\xi|^{\beta-2},|\eta|^{\beta-2}\right\} \operatorname{Re}(\bar{\eta} \xi), \quad \xi, \eta \in \mathbb{C} .
$$

So, to get (2.5) it suffices to obtain

$$
\int_{\mathbb{C}^{2}} \min \left\{|\xi|^{\beta-2},|\eta|^{\beta-2}\right\} \operatorname{Re}(\bar{\eta} \xi) \mathrm{d} \mu(\xi) \mathrm{d} \mu(\eta) \geq 0 .
$$

But this is an immediate consequence of Schur's product theorem for positive definite matrices (in this case we have "continuous" matrices), as both $\min \left\{|\xi|^{\beta-2},|\eta|^{\beta-2}\right\}$ and $\operatorname{Re}(\bar{\eta} \xi)$ express positive definite kernels.

2.5. Scaling and the class of weights. If we keep the confining potential $V$ fixed, and let $n$ (the number of electrons) grow, the process $\Pi_{n}$ will generically grow beyond any confinement. For this reason, it is necessary to jack up the confinement as $n$ grows. This is achieved by putting $V=m Q$, where $m$ is a scaling parameter and

$$
Q: \mathbb{C} \rightarrow \mathbb{R} \cup\{+\infty\}
$$

is a fixed potential, assumed to be lower semi-continuous. To avoid degeneracy, we must suppose that $Q<+\infty$ at least on a set of positive area. From well-known physical considerations, it is natural to let $m$ be essentially proportional to $n$. As we are free to pick $Q$ as we like, we may assume that the proportionality constant is 1 , that is, that $m=n+o(n)$ as $n \rightarrow+\infty$. The growth requirement on $Q$ which conforms with this normalization is

$$
Q(z)-\log |z|^{2} \rightarrow+\infty \quad \text { as }|z| \rightarrow+\infty .
$$


2.6. The equilibrium measure. We consider the limit of the point processes

$$
\Pi_{m Q, n} \quad \text { as } n \rightarrow+\infty \quad \text { while } \frac{n}{m} \rightarrow 1,
$$

while assuming that $Q$ grows in accordance with (2.6). In this case we have convergence of the saddle point configurations. More precisely, the probability measures

$$
\sigma_{n}=\frac{1}{n} \sum_{j} \delta_{z_{j}}
$$

which minimize the functionals (we write $z=\left(z_{1}, \ldots, z_{n}\right)$ )

$$
I_{m Q, n}^{\#}\left[\sigma_{n}\right]:=\frac{2}{n(n-1)} \mathcal{E}_{m Q}(z)=\frac{1}{n(n-1)} \sum_{j, k: j \neq k} \log \frac{1}{\left|z_{j}-z_{k}\right|^{2}}+\frac{2 m}{n(n-1)} \sum_{j} Q\left(z_{j}\right),
$$

converge as $n \rightarrow+\infty$ while $m=n+\mathrm{o}(n)$ in the weak-star sense of measures to the unique probability measure $\sigma=\hat{\sigma}_{Q}$ which minimizes the weighted logarithmic energy

$$
I_{Q}[\sigma]:=\int_{\mathbb{C}^{2}} \log \frac{1}{|\xi-\eta|^{2}} \mathrm{~d} \sigma(\xi) \mathrm{d} \sigma(\eta)+2 \int_{\mathbb{C}} Q \mathrm{~d} \sigma .
$$

This comes as no big surprise given the striking similarity of the expressions $I_{m Q, n}^{\#}\left[\sigma_{n}\right]$ and $I_{Q}[\sigma]$. The configuration of points corresponding to a minimizer $\sigma_{n}$ is known as a collection of weighted Fekete points, and the measure $\hat{\sigma}_{Q}$ is called the equilibrium measure. The existence and uniqueness of the minimizing measure $\hat{\sigma}_{Q}$ is due to Frostman. Let $\operatorname{prob}_{c}(\mathbb{C})$ denote the convex body of all compactly supported Borel probability measures on $\mathbb{C}$.

Theorem 2.4 (Frostman). There exists a unique equilibrium measure $\hat{\sigma}=\hat{\sigma}_{Q}$ such that

$$
I_{Q}[\hat{\sigma}]=\inf _{\sigma} I_{Q}[\sigma],
$$

the infimum being taken over all compactly supported probability measures $\sigma$.

For the proof, we refer to [20]. We will write

$$
\gamma(Q):=I_{Q}\left[\hat{\sigma}_{O}\right], \quad \gamma^{*}(Q):=\gamma(Q)-\int_{\mathbb{C}} Q \mathrm{~d} \hat{\sigma}_{Q},
$$

for the (modified) Robin constants involved. Let

$$
L_{Q}(\xi, \eta):=\log \frac{1}{|\xi-\eta|^{2}}+Q(\xi)+Q(\eta)
$$

and observe that for probability measures $\sigma$, we have

$$
I_{Q}[\sigma]=\int_{\mathbb{C}^{2}} L_{Q}(\xi, \eta) \mathrm{d} \sigma(\xi) \mathrm{d} \sigma(\eta)
$$

Next, we introduce the weighted potential

$$
U_{Q}^{\sigma}(\xi)=\int_{\mathbb{C}} L_{Q}(\xi, \eta) \mathrm{d} \sigma(\eta)
$$

and observe that since

$$
I_{Q}[\sigma]=\int_{\mathbb{C}} U_{Q}^{\sigma}(\xi) \mathrm{d} \sigma(\xi)
$$

we expect that the energy minimizer $\sigma=\hat{\sigma}_{Q}$ should have $U_{Q}^{\sigma}$ constant on the support

$$
S=S_{Q}:=\operatorname{supp} \hat{\sigma}_{Q}
$$

and that constant should also equal the minimum value of $U_{Q}^{\sigma}$. We will at times use the notation $\hat{\sigma}_{Q}=\hat{\sigma}[Q]$ and $S_{Q}=S[Q]$. We use q.e. as short-hand for quasi-everywhere. 
Theorem 2.5 (Frostman). The support $S_{Q}$ of the equilibrium measure $\hat{\sigma}_{Q}$ is compact. Moreover, if $\gamma(Q)$ is as in (2.9), then $U_{Q}^{\hat{\sigma}_{Q}} \geq \gamma(Q)$ q.e. on $\mathbb{C}$, while $U_{Q}^{\hat{\sigma}_{Q}} \leq \gamma(Q)$ at each point of $S_{Q}$. The value $\gamma(Q)$ equals the minimal energy $I_{Q}\left[\hat{\sigma}_{Q}\right]$.

For the proof, we refer to [20]. The number $\mathrm{e}^{-\gamma(Q)}$ is said to be the weighted capacity.

In terms of the usual logarithmic potential

$$
U^{\sigma}(\xi)=\int_{\mathbb{C}} \log \frac{1}{|\xi-\eta|^{2}} \mathrm{~d} \sigma(\eta),
$$

we see that for a compactly supported probability measure $\sigma$,

$$
U_{Q}^{\sigma}(\xi)=\int_{\mathbb{C}} L_{Q}(\xi, \eta) \mathrm{d} \sigma(\eta)=U^{\sigma}(\xi)+Q(\xi)+\int_{\mathbb{C}} Q \mathrm{~d} \sigma,
$$

which allows us to write Frostman's Theorem 2.5 in the following form.

Theorem 2.6 (Frostman). The support $S_{Q}$ of the equilibrium measure $\hat{\sigma}_{Q}$ is compact. Moreover, if $\gamma^{*}(Q)$ is as in (2.9), then $U^{\hat{\sigma}_{Q}}+Q \geq \gamma^{*}(Q)$ q.e. on $\mathbb{C}$, while $U^{\hat{\sigma}_{Q}}+Q \leq \gamma^{*}(Q)$ at each point of $S_{Q}$.

Let $\hat{\sigma}_{m Q, n}$ denote the probability measure $\sigma_{n}$ given by (2.7) corresponding to a weighted Fekete point configuration (i.e., a minimizing configuration). The convergence to the global energy minimizing measure is as follows.

Theorem 2.7 (Fekete, Totik). We have the convergence

$$
\hat{\sigma}_{m Q, n} \rightarrow \hat{\sigma}_{Q} \text { as } n \rightarrow+\infty \text { while } m=n+\mathrm{o}(n)
$$

in the weak-star sense of measures. Moreover, we have convergence in energy:

$$
I_{m,, n}^{\#}\left[\hat{\sigma}_{m Q, n}\right] \rightarrow I_{Q}\left[\hat{\sigma}_{Q}\right]=\gamma(Q), \quad \text { as } n \rightarrow+\infty \text { while } m=n+\mathrm{o}(n) .
$$

For the proof, we refer to [20], p. 145.

2.7. Johansson's marginal measure theorem for the plane. For a probability measure $\sigma \in$ $\operatorname{prob}(\mathbb{C})$ and an integer $k=1,2,3, \ldots$, we denote by $\sigma^{\otimes k} \in \operatorname{prob}\left(\mathbb{C}^{k}\right)$ the product measure given by

$$
\mathrm{d} \sigma^{\otimes k}\left(z_{1}, \ldots, z_{k}\right)=\mathrm{d} \sigma\left(z_{1}\right) \cdots \mathrm{d} \sigma\left(z_{k}\right) .
$$

Definition 2.8. We say that $Q$ has extra growth provided that

$$
Q(z) \geq\left(1+\delta_{0}\right) \log \left(1+|z|^{2}\right)-C_{0}, \quad z \in \mathbb{C},
$$

holds for some small but positive value of $\delta_{0}$ and some (positive) real constant $C_{0}$. Moreover, we say that $Q$ is regular provided that it is bounded and continuous in an open neighborhood of $\mathcal{S}_{Q}=\operatorname{supp} \hat{\sigma}_{Q}$.

Theorem 2.9. Suppose $Q$ is regular with extra growth. Then, for every $k=1,2,3, \ldots$, we have the convergence

$$
\Pi_{m Q, n}^{(k)} \rightarrow \hat{\sigma}_{Q}^{\otimes k} \quad \text { as } n \rightarrow+\infty \text { while } m=n+\mathrm{o}(n),
$$

in the weak-star sense of measures.

Remark 2.10. (i) Johansson [17] proves his theorem in the degenerate real line case when $Q(\xi)=+\infty$ for $\xi \in \mathbb{C} \backslash \mathbb{R}$ (the Hermitian matrix case). This can be viewed as a limit case of our considerations. However, the approach of Johansson's proof can be modified so as to include the complex plane case stated here. We indicate the necessary modifications in an appendix below.

(ii) An alternative formulation of Theorem 2.9 runs as follows. As $n \rightarrow+\infty$ while $m=n+\mathrm{o}(n)$, the random variables $z_{1}, \ldots, z_{k}$ on $\left(\mathbb{C}^{n}, \Pi_{m Q, n}\right)$ are asymptotically i.i.d. with law $\hat{\sigma}_{Q}$. 
(iii) We now find an application of Theorem 2.9 to linear statistics. Let $C_{b}\left(\mathbb{C}^{k}\right)$ denote the Banach space of bounded continuous functions in $\mathbb{C}^{k}$. Moreover, let the $\operatorname{trace} \operatorname{tr}_{n} f$ of the function $f \in \mathbb{C}_{b}(\mathbb{C})$ be given by

$$
\operatorname{tr}_{n} f=\sum_{j} f\left(z_{j}\right)
$$

where the sum as usual runs over $j=1, \ldots, n$ and $z_{1}, \ldots, z_{n}$ are random variables with joint probability $\left(\mathbb{C}^{n}, \Pi_{m Q, n}\right)$. For each $j=1, \ldots, n$, we have, in view of Johansson's marginal measure theorem, for $f \in \mathrm{C}_{b}(\mathbb{C})$,

$$
\mathbb{E}\left[f\left(z_{j}\right)\right]=\int_{\mathbb{C}} f(\xi) \mathrm{d} \Pi_{m Q, n}^{(1)}(\xi) \rightarrow \int_{\mathbb{C}} f(\xi) \mathrm{d} \hat{\sigma}_{Q}(\xi)=:\left\langle f, \hat{\sigma}_{Q}\right\rangle,
$$

as $n \rightarrow+\infty$ while $m=n+\mathrm{o}(n)$. By forming the average over $j$, we get, for $f \in \mathrm{C}_{b}(\mathbb{C})$,

$$
\mathbb{E}\left[\frac{1}{n} \operatorname{tr}_{n} f\right]=\int_{\mathbb{C}} f(\xi) \mathrm{d} \Pi_{m Q, n}^{(1)}(\xi) \rightarrow\left\langle f, \hat{\sigma}_{Q}\right\rangle,
$$

as $n \rightarrow+\infty$ while $m=n+\mathrm{o}(n)$. There is an analogous statement which holds for functions $f \in \mathrm{C}_{b}\left(\mathbb{C}^{k}\right)$ and involves the measure $\hat{\sigma}_{Q}^{\otimes k}$ in place of $\hat{\sigma}_{Q}$. This more general statement allows us to obtain that for $f \in \mathrm{C}_{b}(\mathbb{C}),\left(k, k^{\prime}\right.$ are fixed integers $\left.\geq 0\right)$

$$
\mathbb{E}\left[\left(\frac{1}{n} \operatorname{tr}_{n} f\right)^{k}\left(\frac{1}{n} \operatorname{tr}_{n} \bar{f}\right)^{k^{\prime}}\right] \rightarrow\left(\left\langle f, \hat{\sigma}_{Q}\right\rangle\right)^{k}\left(\left\langle\bar{f}, \hat{\sigma}_{Q}\right\rangle\right)^{k^{\prime}},
$$

as $n \rightarrow+\infty$ while $m=n+\mathrm{o}(n)$. Here, as usual, $\bar{f}$ is the function whose values are complex conjugate to those of $f$. This expresses that $\frac{1}{n} \operatorname{tr}_{n} f$ tends to the constant value $\left\langle f, \hat{\sigma}_{Q}\right\rangle$ in all moments as $n \rightarrow+\infty$ while $m=n+\mathrm{o}(n)$, and hence in particular, we have convergence in distribution (as in the weak law of large numbers).

(iv) We remark that Theorem 2.9 holds independently of the value of the inverse temperature $\beta$. However, for $\beta=2$, much more precise statements have been obtained recently in [1], [2], [3]. The reason why this is possible is the determinantal property (2.2). To give some hints about the results, we introduce the fluctuation

$$
\mathrm{fl}_{n} f=\operatorname{tr}_{n} f-n\left\langle f, \hat{\sigma}_{Q}\right\rangle .
$$

In view of (iv), we know that $\frac{1}{n} \mathrm{fl}_{n} f \rightarrow 0$ in moments and hence in distribution as $n \rightarrow+\infty$ while $m=n+\mathrm{o}(n)$. Next, suppose $n \rightarrow+\infty$ while $m=n+\mathrm{o}(1)$, which means that $m$ is kept much closer to $n$ than before, and suppose also that the function $Q$ is real-analytically smooth with $\Delta Q>0$ in the interior of $S_{Q}$ (we recall that $S_{Q}$ is the support of the equilibrium measure $\hat{\sigma}_{Q}$ ). In analogy with the CLT (central limit theorem), it is shown in [1], [2] that under some additional assumptions, the stochastic variable $\mathrm{fl}_{n} f$ converges in distribution to a real-valued Gaussian with expectation $e_{f}$ and variance $v_{f}$,

$$
e_{f}=\frac{1}{2 \pi} \int_{S_{Q}} f \Delta \log \Delta Q \mathrm{dvol}_{2}, \quad v_{f}=\frac{1}{4 \pi} \int_{S_{Q}}|\nabla f|^{2} \mathrm{dvol}_{2},
$$

provided the function $f$ is real-valued, $C^{\infty}$-smooth, and is supported in the interior of $S_{Q}$. The extension to general test functions $f$ is obtained in [3]; the general formulae for $e_{f}, v_{f}$ include boundary effects.

2.8. Johansson's free energy theorem for the plane. We recall the expression for the normalization constant

$$
Z_{m, n}=\int_{\mathbb{C}^{n}}|\triangle(z)|^{\beta} \mathrm{e}^{-\frac{\beta}{2} m \sum_{j} Q\left(z_{j}\right)} \mathrm{dvol}_{2 n}(z),
$$


which we write in the form

$$
Z_{m, n}=\int_{\mathbb{C}^{n}} \exp \left\{-\frac{\beta}{4} \sum_{j, k: j \neq k} L_{Q}\left(z_{j}, z_{k}\right)+\frac{\beta}{2}(n-m-1) \sum_{j} Q\left(z_{j}\right)\right\} \mathrm{dvol}_{2 n}(z),
$$

where $L_{Q}$ is as in (2.10). The quantity

$$
\frac{1}{n(n-1)} \log Z_{m, n}
$$

has in the physics literature acquired the name free energy (frequently $n^{2}$ is used in place of $n(n-1)$; asymptotically, there is no difference). See Definition 2.8 for the terms regular and extra growth.

Theorem 2.11. Suppose $Q$ is regular with extra growth. Then

$$
\frac{1}{n(n-1)} \log Z_{m, n} \rightarrow-\frac{\beta}{4} \gamma(Q)=-\frac{\beta}{4} I_{Q}\left[\hat{\sigma}_{Q}\right] \text { as } n \rightarrow+\infty \text { while } m=n+\mathrm{o}(n) \text {. }
$$

Remark 2.12. As with Theorem 2.9. Johansson [17] proves his theorem in the degenerate real line case when $Q(\xi)=+\infty$ for $\xi \in \mathbb{C} \backslash \mathbb{R}$ (the Hermitian matrix case). This can be viewed as a limit case of our considerations. However, the approach of Johansson's proof can be modified so as to include the complex plane case stated here. We indicate the necessary modifications in an appendix below.

2.9. Aggregation of equilibrium measures. We now look at the quasi-classical limit of the evolution of quantum droplets (the addition of more electrons to the droplet). This will allow us to understand how the quantum process is related to a growth process of Hele-Shaw type for compact sets in the plane.

We restrict our attention to the potentials $Q$ that satisfy a scale invariant version of the growth condition (2.6), namely

$$
Q(z)-A \log |z| \rightarrow+\infty, \quad \text { as }|z| \rightarrow+\infty,
$$

no matter how big the positive parameter $A$ gets. We will be interested in the evolution of positive measures

$$
\hat{\sigma}_{t} \equiv \hat{\sigma}_{t}[Q]:=t \hat{\sigma}_{Q / t},
$$

where $t$ ranges over $0<t<+\infty$. We write $S_{t}=S_{t}[Q]$ for the support of the measure $\hat{\sigma}_{t}[Q]$ (i.e., $\left.S_{t}=S_{Q / t}\right)$. Note that $\hat{\sigma}_{t}[Q]$ has total mass $t$. The process of increasing the parameter $t$ has the following interpretation. We consider the limit process of letting $n \rightarrow+\infty$ while $m=n / t+o(n)$. In other words, $m \rightarrow+\infty$ while $n=m t+\mathrm{o}(m)$. An increase of $t$ therefore has the interpretation of increasing the total number of electrons $n$ for fixed $m$. To rescale, we introduce $m^{\prime}=m t$, so that the relationship reads $n=m^{\prime}+\mathrm{o}\left(m^{\prime}\right)$. Since $m Q=m^{\prime} Q / t$, rescaling also means we must replace $Q$ by $Q / t$. In view of Johansson's theorem, we find that

$$
\lim \mathbb{E} \frac{\#\{\text { electrons in } e\}}{m}=t \lim \mathbb{E} \frac{\#\{\text { electrons in } e\}}{m^{\prime}}=t \hat{\sigma}_{Q / t}(e)=\hat{\sigma}_{t}[Q](e) .
$$

In other words, the growth process $\hat{\sigma}_{t}[Q]$ is the quasi-classical limit of the growth process of adding electrons to the quantum droplet. We shall see that if $Q$ is $C^{2}$-smooth, the measure $\hat{\sigma}_{t}[Q]$ is determined uniquely by its support $S_{t}[Q]$. We understand the set $S_{t}[Q]$ as a (classical) droplet; cf. Subsection 5.1 for a precise definition.

Corollary 2.13. The family of measures $\hat{\sigma}_{t}[Q]$ is monotonically increasing in $t$.

Proof. This is true for quantum droplets if $\beta=2$; the quasi-classical limit does not depend on $\beta$.

Remark 2.14. It is not hard to write down a potential theoretic proof of this fact; see Proposition 4.15 . 
3. Appendix: PRoof of Johansson's marginAl MEASURE AND FREE ENERGY THEOREMS FOR THE PLANE

3.1. Fekete configurations. The approach to prove Johansson's theorem in this setting is to show that point configurations $\left(z_{1}, \ldots, z_{n}\right)$ whose associated energy functional

$$
I_{n,(n-1) Q}^{\sharp}\left[\sigma_{n}\right]=\frac{1}{n(n-1)} \sum_{j, k: j \neq k} L_{Q}\left(z_{j}, z_{k}\right)
$$

deviate substantially from the minimum are highly unlikely. We note that since $m=n+\mathrm{o}(n)$ is assumed, the choice to replace $m$ by $n-1$ in the energy is reasonable. Let write

$$
\hat{\sigma}_{n}:=\hat{\sigma}_{n,(n-1) Q}
$$

for the minimizing (Fekete) measure in the context of Theorem 2.7 and we also write

$$
I_{n}^{\sharp}\left[\hat{\sigma}_{n}\right]:=I_{n,(n-1) Q}^{\sharp}\left[\hat{\sigma}_{n,(n-1) Q}\right]
$$

for the associated energy. By [20], pp. 143-145, the sequence of energies $I_{n}^{\sharp}\left[\hat{\sigma}_{n}\right]$ is decreasing in $n$, and converges to $I_{Q}\left[\hat{\sigma}_{Q}\right]=\gamma(Q)$ as $n \rightarrow+\infty$ (cf. Theorem 2.7).

3.2. An entropy estimate. We introduce an auxiliary Borel measurable function $\phi: \mathbb{C} \rightarrow[0,+\infty)$ with

$$
\int_{\mathbb{C}} \phi \mathrm{dvol}_{2}=1, \quad \int_{\mathbb{C}}(Q+|\log \phi|) \phi \mathrm{dvol}_{2}<+\infty,
$$

with the understanding that $\phi \log \phi=0$ at points where $\phi=0$. We sort of artificially smuggle it into the expression (2.14) for $Z_{m, n}$ :

$$
Z_{m, n}=\int_{\mathbb{C}^{n}} \exp \left\{-\frac{\beta}{4} \sum_{j, k: j \neq k} L_{Q}\left(z_{j}, z_{k}\right)+\frac{\beta}{2}(n-m-1) \sum_{j} Q\left(z_{j}\right)-\sum_{j} \log \phi\left(z_{j}\right)\right\} \prod_{j} \phi\left(z_{j}\right) \mathrm{dvol}_{2 n}(z) .
$$

Now, by Jensen's inequality, we have, with $\mathrm{d} \sigma_{\phi}=\phi \mathrm{dvol}_{2}$,

(3.2) $\log Z_{m, n}$

$$
\begin{array}{r}
\geq \log \int_{\mathbb{C}^{n}}\left\{-\frac{\beta}{4} \sum_{j, k: j \neq k} L_{Q}\left(z_{j}, z_{k}\right)+\frac{\beta}{2}(n-m-1) \sum_{j} Q\left(z_{j}\right)-\sum_{j} \log \phi\left(z_{j}\right)\right\} \prod_{j} \phi\left(z_{j}\right) \mathrm{dvol}_{2 n}(z) \\
=-\frac{\beta n(n-1)}{4} \int_{\mathbb{C}^{2}} L_{Q}(\xi, \eta) \mathrm{d} \sigma_{\phi}(\xi) \mathrm{d} \sigma_{\phi}(\eta)+\frac{\beta}{2} n(n-m-1) \int_{\mathbb{C}} Q \mathrm{~d} \sigma_{\phi}-n \int_{\mathbb{C}} \log \phi \mathrm{d} \sigma_{\phi},
\end{array}
$$

where we used repeatedly that $\sigma_{\phi}$ is a probability measure. We rewrite this as

$$
\frac{1}{n(n-1)} \log Z_{m, n} \geq-\frac{\beta}{4} I_{Q}\left[\sigma_{\phi}\right]+\beta \frac{n-m-1}{2(n-1)} \int_{\mathbb{C}} Q \mathrm{~d} \sigma_{\phi}-\frac{1}{n-1} \int_{\mathbb{C}} \log \phi \mathrm{d} \sigma_{\phi},
$$

This gives (as $n \rightarrow+\infty$ while $m=n+\mathrm{o}(n))$

$$
\liminf \frac{1}{n(n-1)} \log Z_{m, n} \geq-\frac{\beta}{4} I_{Q}\left[\sigma_{\phi}\right] .
$$

The condition on $\phi$ that $\phi \log \phi \in L^{1}(\mathbb{C})$ is of entropy type, and this is the reason why we call (3.3) an entropy estimate. We would like to plug in the choice $\sigma_{\phi}=\hat{\sigma}_{Q}$ into the entropy estimate (3.3) to get an effective bound. At this point, we do not know enough about $\hat{\sigma}_{Q}$ to be sure whether it is of the form $\sigma_{\phi}$ with $\phi$ meeting (3.1). To remedy this, we consider the function $\phi_{r}: \mathbb{C} \rightarrow[0,+\infty)$ given by $(0<r \leq 1)$

$$
\phi_{r}(\xi)=\frac{1}{\pi r^{2}} \int_{\mathbb{D}(\xi, r)} \mathrm{d} \hat{\sigma}_{Q}(\eta)=\frac{1}{\pi r^{2}} \int_{\tau \in \mathbb{D}(0, r)} \mathrm{d} \hat{\sigma}_{Q}(\xi-\tau)
$$


this amounts to convolution with the normalized characteristic function of the disk $\mathbb{D}(0, r)$. The corresponding measure

$$
\mathrm{d} \sigma_{r}:=\mathrm{d} \sigma_{\phi_{r}}=\phi_{r} \mathrm{dvol}_{2}
$$

is a compactly supported (Borel) probability measure, with density $\phi_{r} \in L^{\infty}(\mathbb{C})$, so that (3.1) holds with $\phi_{r}$ in place of $\phi$. By the standard properties of convolutions, $\sigma_{r} \rightarrow \hat{\sigma}_{Q}$ in the weak-star sense of measures as $r \rightarrow 0$. We claim that we also have convergence in energy,

$$
I_{Q}\left[\sigma_{r}\right] \rightarrow I_{Q}\left[\hat{\sigma}_{Q}\right]=\gamma(Q) \text { as } r \rightarrow 0 .
$$

Suppose for the moment that we have obtained (3.4). Then we find from the approximation procedure that

$$
\liminf \frac{1}{n^{2}} \log Z_{m, n} \geq-\frac{\beta}{4} I_{Q}\left[\hat{\sigma}_{Q}\right]=-\frac{\beta}{4} \gamma(Q) .
$$

To obtain (3.4), we note that interchanging the order of integration gives

$$
I_{Q}\left[\sigma_{r}\right]-I_{Q}\left[\hat{\sigma}_{Q}\right]=2 \int_{\mathbb{C}} Q\left(\mathrm{~d} \sigma_{r}-\mathrm{d} \hat{\sigma}_{Q}\right)+\int_{\mathbb{C}^{2}} \Lambda_{r}(\xi, \eta) \mathrm{d} \hat{\sigma}_{Q}(\xi) \mathrm{d} \hat{\sigma}_{Q}(\eta),
$$

where

$$
\Lambda_{r}(\xi, \eta)=\frac{2}{\pi^{2} r^{4}} \int_{\left(\tau, \tau^{\prime}\right) \in \mathbb{D}\left(0, r^{2}\right.}\left[\log \left|(\xi+\tau)-\left(\eta+\tau^{\prime}\right)\right|-\log |\xi-\eta|\right] \operatorname{dvol}_{2}(\tau) \operatorname{dvol}_{2}\left(\tau^{\prime}\right) .
$$

The support of $\sigma_{r}$ is at most within distance $r$ from the support $\mathcal{S}_{Q}$ of $\hat{\sigma}_{Q}$, so in view of the assumption that $Q$ be bounded and continuous in a fixed neighborhood of $\mathcal{S}_{Q}$, we get

$$
\int_{\mathbb{C}} Q\left(\mathrm{~d} \sigma_{r}-\mathrm{d} \hat{\sigma}_{Q}\right)=\int_{\mathbb{C}} Q \mathrm{~d} \sigma_{r}-\int_{\mathbb{C}} Q \mathrm{~d} \hat{\sigma}_{Q} \rightarrow 0 \text { as } r \rightarrow 0 .
$$

Next, we rewrite the expression for $\Lambda_{r}$ :

$$
\begin{aligned}
\Lambda_{r}(\xi, \eta)=\frac{2}{\pi^{2} r^{4}} \int_{\left(\tau, \tau^{\prime}\right) \in \mathbb{D}(0, r)^{2}} & \log \left|1+\frac{\tau-\tau^{\prime}}{\xi-\eta}\right| \operatorname{dvol}_{2}(\tau) \operatorname{dvol}_{2}\left(\tau^{\prime}\right) \\
& =\frac{2}{\pi^{2} r^{4}} \int_{\tau^{\prime \prime} \in \mathbb{D}(0,2 r)} \operatorname{vol}_{2}\left(\mathbb{D}(0, r) \cap \mathbb{D}\left(\tau^{\prime \prime}, r\right)\right) \log \left|1+\frac{\tau^{\prime \prime}}{\xi-\eta}\right| \operatorname{dvol}_{2}\left(\tau^{\prime \prime}\right) .
\end{aligned}
$$

We use that the common area of the two intersecting circular disks is

$$
\operatorname{vol}_{2}\left(\mathbb{D}(0, r) \cap \mathbb{D}\left(\tau^{\prime \prime}, r\right)\right)=2 r^{2} \arccos \frac{\left|\tau^{\prime \prime}\right|}{2 r}-r\left|\tau^{\prime \prime}\right| \sqrt{1-\frac{\left|\tau^{\prime \prime}\right|^{2}}{4 r^{2}}}
$$

to get

$$
\Lambda_{r}(\xi, \eta)=\frac{4}{\pi^{2} r^{2}} \int_{\tau^{\prime \prime} \in \mathbb{D}(0,2 r)}\left\{\arccos \frac{\left|\tau^{\prime \prime}\right|}{2 r}-\frac{\left|\tau^{\prime \prime}\right|}{2 r} \sqrt{1-\frac{\left|\tau^{\prime \prime}\right|^{2}}{4 r^{2}}}\right\} \log \left|1+\frac{\tau^{\prime \prime}}{\xi-\eta}\right| \operatorname{dvol}_{2}\left(\tau^{\prime \prime}\right) .
$$

The identity

$$
\int_{-\pi}^{\pi} \log \left|1+\lambda \mathrm{e}^{\mathrm{i} \theta}\right| \mathrm{d} \theta=2 \pi \log ^{+}|\lambda|, \quad \lambda \in \mathbb{C},
$$

where for real $x \geq 0, \log ^{+} x=\max \{0, \log x\}$, shows that

$$
\Lambda_{r}(\xi, \eta)=0, \quad \text { if } 2 r \leq|\xi-\eta|,
$$

while

$$
\Lambda_{r}(\xi, \eta)=\frac{8}{\pi r^{2}} \int_{|\xi-\eta|}^{2 r}\left\{\arccos \frac{s}{2 r}-\frac{s}{2 r} \sqrt{1-\frac{s^{2}}{4 r^{2}}}\right\} \log \frac{s}{|\xi-\eta|} s \mathrm{~d} s \quad \text { if }|\xi-\eta|<2 r .
$$


In the latter case, we may use that for $|\xi-\eta|<s<2 r$,

$$
0 \leq \arccos \frac{s}{2 r}-\frac{s}{2 r} \sqrt{1-\frac{s^{2}}{4 r^{2}}} \leq \frac{\pi}{2}, \quad 0 \leq \log \frac{s}{|\xi-\eta|} \leq \log \frac{2 r}{|\xi-\eta|},
$$

to conclude that

$$
0 \leq \Lambda_{r}(\xi, \eta) \leq 8 \log \frac{2 r}{|\xi-\eta|} \text { if }|\xi-\eta|<2 r .
$$

It follows that generally, we have

$$
0 \leq \Lambda_{r}(\xi, \eta) \leq 8 \log ^{+} \frac{2 r}{|\xi-\eta|} .
$$

The measure $\hat{\sigma}_{Q}$ has compact support and finite logarithmic energy,

$$
\int_{\mathbb{C}^{2}} \log \frac{1}{|\xi-\eta|} \mathrm{d} \hat{\sigma}_{Q}(\xi) \mathrm{d} \hat{\sigma}_{Q}(\eta)<+\infty,
$$

so that if we use (3.7) and the Lebesgue's domintated convergence theorem, we see that

$$
\int_{\mathbb{C}^{2}} \Lambda_{r}(\xi, \eta) \mathrm{d} \hat{\sigma}_{Q}(\xi) \mathrm{d} \hat{\sigma}_{Q}(\eta) \rightarrow 0 \quad \text { as } r \rightarrow 0 .
$$

As we combine this with (3.6), the claimed energy convergence (3.4) is immediate, and hence (3.5) follows.

3.3. Low probability of high energy configurations. In view of (3.5), we have

$$
\frac{1}{n(n-1)} \log Z_{m, n} \geq-\frac{\beta}{4}(\gamma(Q)+\varepsilon),
$$

for fixed positive $\varepsilon$ and large enough $n$. In this context, we think of $m=m_{n}$ as (fixed) sequence which depends on $n$, with $m=m_{n}=n+\mathrm{o}(n)$.

We put

$$
G(\xi, \eta):=\log \frac{\left(1+|\xi|^{2}\right)\left(1+|\eta|^{2}\right)}{|\xi-\eta|^{2}} \geq 0, \quad \xi, \eta \in \mathbb{C} .
$$

In view of the assumed extra growth (2.13), we have

$$
\begin{aligned}
& L_{Q}(\xi, \eta)=\log \frac{1}{|\xi-\eta|^{2}}+Q(\xi)+Q(\eta) \\
& \qquad \begin{array}{l}
\geq \log \frac{1}{|\xi-\eta|^{2}}+\frac{\delta_{0}}{1+\delta_{0}}[Q(\xi)+Q(\eta)]+\log \left[\left(1+|\xi|^{2}\right)\left(1+|\eta|^{2}\right)\right]-\frac{2 C_{0}}{1+\delta_{0}} \\
\quad=G(\xi, \eta)+\frac{\delta_{0}}{1+\delta_{0}}[Q(\xi)+Q(\eta)]-\frac{2 C_{0}}{1+\delta_{0}}
\end{array}
\end{aligned}
$$

where $\delta_{0}$ and $C_{0}$ are as in (2.13). To simplify the notation, we write, with $z=\left(z_{1}, \ldots, z_{n}\right)$,

$$
L_{Q}^{\langle\langle\eta\rangle}(z)=\sum_{j, k: j \neq k} L_{Q}\left(z_{j}, z_{k}\right), \quad \text { and } \quad G^{\langle n\rangle}(z)=\sum_{j, k: j \neq k} G\left(z_{j}, z_{k}\right) \geq 0,
$$

where it is assumed that $j$ and $k$ range over $\{1, \ldots, n\}$. These expressions are of "double trace type" associated with the functions $L_{Q}$ and $G$ (see (2.10) and (3.9)). We also have the "trace type" expressions (with $z=\left(z_{1}, \ldots, z_{n}\right)$ )

$$
Q^{\langle n\rangle}(z)=\sum_{j} Q\left(z_{j}\right) \quad \text { and } \quad \Lambda^{\langle n\rangle}(z):=\sum_{j} \log \left(1+\left|z_{j}\right|^{2}\right) .
$$

It now follows from (3.10) that

$$
L_{Q}^{\langle n\rangle\rangle}(z) \geq G^{\langle n\rangle\rangle}(z)+\frac{2 \delta_{0}(n-1)}{1+\delta_{0}} Q^{\langle n\rangle}(z)-\frac{2 C_{0}}{1+\delta_{0}} n(n-1),
$$


while a direct application of the extra growth condition (2.13) leads to

$$
Q^{\langle n\rangle}(z) \geq\left(1+\delta_{0}\right) \Lambda^{\langle n\rangle}(z)-C_{0} n .
$$

The point with introducing this notation is that (2.14) simplifies to

$$
Z_{m, n}=\int_{\mathbb{C}^{n}} \exp \left\{-\frac{\beta}{4} L_{Q}^{\langle n\rangle\rangle}(z)+\frac{\beta}{2}(n-m-1) Q^{\langle n\rangle}(z)\right\} \mathrm{dvol}_{2 n}(z),
$$

while the probability density becomes

$$
\mathrm{d} \Pi_{m Q, n}(z)=\frac{1}{Z_{m, n}} \exp \left\{-\frac{\beta}{4} L_{Q}^{\langle\langle n\rangle}(z)+\frac{\beta}{2}(n-m-1) Q^{\langle n\rangle}(z)\right\} \mathrm{dvol}_{2 n}(z),
$$

As mentioned in Subsection 3.1, we have the estimate

$$
\frac{1}{n(n-1)} L_{Q}^{\langle n\rangle}(z) \geq \gamma(Q), \quad z=\left(z_{1}, \ldots, z_{n}\right) \in \mathbb{C}^{n} .
$$

We introduce the set

$$
\mathcal{A}(n, \epsilon)=\left\{z \in \mathbb{C}^{n}: \frac{1}{n(n-1)} L_{Q}^{\langle n\rangle}(z) \leq \gamma(Q)+\epsilon\right\},
$$

where $\epsilon$ is a positive real number.

Proposition 3.1. There exists a positive integer $N_{0}$, which depends on $\epsilon>0$ but not on $a \geq 0$, such that

$$
\Pi_{m Q, n}\left(\mathbb{C}^{n} \backslash \mathcal{A}(n, \epsilon+a)\right) \leq \mathrm{e}^{-\beta a n(n-1) / 8}, \quad n \geq N_{0},
$$

provided the sequence $m=m_{n}=n+\mathrm{o}(n)$ is kept fixed.

Proof. By definition, we have

$$
\frac{1}{n(n-1)} L_{Q}^{\langle n\rangle)}(z)>\gamma(Q)+\epsilon+a, \quad z \in \mathbb{C}^{n} \backslash \mathcal{A}(n, \epsilon+a) .
$$

We rewrite (3.11) as

$$
\frac{1}{n(n-1)} L_{Q}^{\langle n\rangle}(z) \geq \frac{2 \delta_{0}}{\left(1+\delta_{0}\right) n} Q^{\langle n\rangle}(z)-\frac{2 C_{0}}{1+\delta_{0}}, \quad z \in \mathbb{C}^{n},
$$

and form a convex combination of (3.17) and (3.18) (we keep $\theta$ fixed with $0<\theta<1$ )

$$
\frac{1}{n(n-1)} L_{Q}^{\langle n\rangle}(z) \geq(1-\theta)(\gamma(Q)+\epsilon+a)+\frac{\theta}{1+\delta_{0}}\left\{\frac{2 \delta_{0}}{n} Q^{\langle n\rangle}(z)-2 C_{0}\right\}, \quad z \in \mathbb{C}^{n} \backslash \mathcal{A}(n, \epsilon+a) .
$$

The exponent in the density defining $\Pi_{m Q, n}$ is (cf. (3.13)

$$
-\frac{\beta}{4} \sum_{j, k: j \neq k} L_{Q}\left(z_{j}, z_{k}\right)+\frac{\beta}{2}(n-m-1) \sum_{j} Q\left(z_{j}\right)=-\frac{\beta}{4} L_{Q}^{\langle n\rangle\rangle}(z)+\frac{\beta}{2}(n-m-1) Q^{\langle n\rangle}(z),
$$

and in view of the estimate (3.19) we get

$$
\begin{aligned}
-\frac{\beta}{4} L_{Q}^{\langle n\rangle\rangle}(z)+\frac{\beta}{2}(n-m-1) Q^{\langle n\rangle}(z) \leq-\frac{\beta}{4} n(n-1)(1-\theta)(\gamma(Q)+\epsilon+a) & \\
& -\frac{\beta}{2}\left\{\theta(n-1) \frac{\delta_{0}}{1+\delta_{0}}-(n-m-1)\right\} Q^{\langle n\rangle}(z)+\frac{C_{1} \theta \beta}{4} n(n-1), \quad z \in \mathbb{C}^{n} \backslash \mathcal{A}(n, \epsilon+a) .
\end{aligned}
$$

If

$$
\frac{m}{n-1}>1-\frac{\theta \delta_{0}}{1+\delta_{0}}
$$


holds, which is bound to be the case for big enough $n$ (provided $\theta$ is kept away from 0 ), since $m=n+\mathrm{o}(n)$, the expression in front of $Q^{\langle n\rangle}(z)$ on the right hand side of $(3.20)$ is negative, and we may apply (3.12) to (3.20), and arrive at

$$
\begin{aligned}
- & \frac{\beta}{4} L_{Q}^{\langle n\rangle\rangle}(z)+\frac{\beta}{2}(n-m-1) Q^{\langle n\rangle}(z) \\
\leq-\frac{\beta}{4} n(n-1)(1-\theta)(\gamma(Q) & +\epsilon+a)-\frac{\beta}{2}\left\{\theta(n-1) \delta_{0}-\left(1+\delta_{0}\right)(n-m-1)\right\} \Lambda^{\langle n\rangle}(z) \\
& +\frac{C_{0} \beta \theta}{2} n(n-1)-\frac{C_{0} \beta}{2} n(n-m-1), \quad z \in \mathbb{C}^{n} \backslash \mathcal{A}(n, \epsilon+a) .
\end{aligned}
$$

As a consequence, we find that

$$
\begin{aligned}
& \Pi_{m Q, n}\left(\mathbb{C}^{n} \backslash \mathcal{A}(n, \epsilon+a)\right)=\frac{1}{Z_{m, n}} \int_{\mathbb{C}^{n} \backslash \mathcal{A}(n, \epsilon+a)} \exp \left\{-\frac{\beta}{4} L_{Q}^{\langle n\rangle}(z)+\frac{\beta}{2}(n-m-1) Q^{\langle n\rangle}(z)\right\} \operatorname{dvol}_{2 n}(z) \\
& \leq \frac{1}{Z_{m, n}} \exp \left\{-\frac{\beta}{4} n(n-1)(1-\theta)(\gamma(Q)+\right.\left.\epsilon+a)+\frac{C_{0} \beta \theta}{2} n(n-1)-\frac{C_{0} \beta}{2} n(n-m-1)\right\} \\
& \times\left\{\int_{\mathbb{C}}\left(1+|\xi|^{2}\right)^{-\frac{\beta}{2}\left\{\theta(n-1) \delta_{0}-\left(1+\delta_{0}\right)(n-m-1)\right\}} \operatorname{dvol}_{2}(\xi)\right\}^{n} .
\end{aligned}
$$

An exercise involving polar coordinates convinces us that for $\alpha>1$,

$$
\int_{\mathbb{C}}\left(1+|\xi|^{2}\right)^{-\alpha} \operatorname{dvol}_{2}(\xi)=\frac{\pi}{\alpha-1},
$$

and we see that (3.23) entails that

$$
\begin{aligned}
& \Pi_{m Q, n}\left(\mathbb{C}^{n} \backslash \mathcal{A}(n, \epsilon+a)\right) \\
& \leq \frac{1}{Z_{m, n}} \exp \left\{-\frac{\beta}{4} n(n-1)(1-\theta)(\gamma(Q)+\epsilon+\right.\left.a)+\frac{C_{0} \beta \theta}{2} n(n-1)-\frac{C_{0} \beta}{2} n(n-m-1)\right\} \\
& \times\left\{\frac{2 \pi}{\beta\left\{\theta(n-1) \delta_{0}-\left(1+\delta_{0}\right)(n-m-1)\right\}-2}\right\}^{n},
\end{aligned}
$$

provided that

$$
\frac{m}{n-1}>1-\frac{\theta \delta_{0}}{1+\delta_{0}}+\frac{2}{\beta\left(1+\delta_{0}\right)(n-1)} .
$$

Let us assume slightly more, namely that

$$
\frac{m}{n-1}>1-\frac{\theta \delta_{0}}{1+\delta_{0}}+\frac{2(1+\pi)}{\beta\left(1+\delta_{0}\right)(n-1)}
$$

which is a little stronger than (3.21), and holds for big enough $n$ (as long as $\theta$ is kept away from $0)$, since $m=n+o(n)$. This allows us to get rid of the last factor in the right hand side of (3.25):

$$
\begin{aligned}
\Pi_{m Q, n} & \left(\mathbb{C}^{n} \backslash \mathcal{A}(n, \epsilon+a)\right) \\
& \leq \frac{1}{Z_{m, n}} \exp \left\{-\frac{\beta}{4} n(n-1)(1-\theta)(\gamma(Q)+\epsilon+a)+\frac{C_{0} \beta \theta}{2} n(n-1)-\frac{C_{0} \beta}{2} n(n-m-1)\right\} .
\end{aligned}
$$

We finally implement the estimate (3.8), and get

$$
\begin{aligned}
\prod_{m Q, n}\left(\mathbb{C}^{n} \backslash \mathcal{A}(n, \epsilon+a)\right) & \\
& \leq \exp \left\{\frac{\beta}{4} n(n-1)\left[\theta \gamma(Q)-(1-\theta)(\epsilon+a)+\varepsilon+2 \theta C_{0}-2 C_{0}\left(1-\frac{m}{n-1}\right)\right]\right\} .
\end{aligned}
$$


The constant $C_{0}$ is assumed positive, and we may therefore pick a small $\theta, 0<\theta<\frac{1}{2}$, such that

$$
\theta\left[\gamma(Q)+2 C_{0}\right] \leq \frac{\epsilon}{2}
$$

Since $m=n+\mathrm{o}(n)$, it follows from (3.28) that

$$
\left.\Pi_{m Q, n}\left(\mathbb{C}^{n} \backslash \mathcal{A}(n, \epsilon+a)\right) \leq \exp \left\{\frac{\beta}{4} n(n-1)\left[-(1-\theta) a-\left(\frac{1}{2}-\theta\right) \epsilon+\varepsilon+\mathrm{o}(1)\right)\right]\right\} .
$$

Also, by choosing $\varepsilon$ sufficiently small, we can make sure that

$$
-\left(\frac{1}{2}-\theta\right) \epsilon+\varepsilon+o(1) \leq 0
$$

for big $n$, so that (3.29) gives

$$
\Pi_{m Q, n}\left(\mathbb{C}^{n} \backslash \mathcal{A}(n, \epsilon+a)\right) \leq \exp \left\{-\frac{\beta}{4}(1-\theta) a n(n-1)\right\} \leq \exp \left\{-\frac{\beta}{8} a n(n-1)\right\},
$$

as claimed.

3.4. The proof of Johansson's free energy theorem. The claim is that

$$
\frac{1}{n(n-1)} \log Z_{m, n} \rightarrow-\frac{\beta}{4} \gamma(Q) \quad \text { as } n \rightarrow+\infty \text { while } m=n+o(n) .
$$

Note that by (3.5) we only need to show that lim sup converges to a number $\leq-\beta \gamma(Q) / 4$. To this end, we begin by establishing that for $0<\theta<1$, we have

$$
\begin{aligned}
-\frac{1}{2} L_{Q}^{\langle n\rangle}(z)+ & (n-m-1) Q^{\langle n\rangle}(z) \\
\leq & -\frac{1-\theta}{2} n(n-1) \gamma(Q)-\left[(n-1) \frac{\delta_{0} \theta}{1+\delta_{0}}-(n-m-1)\right] Q^{\langle n\rangle}(z)+\frac{C_{1} \theta}{2} n(n-1),
\end{aligned}
$$

by forming a convex combination of (3.15) and (3.18). By applying (3.12) to (3.32), we get that (since the expression in front of $Q^{\langle n\rangle}(z)$ is negative for big $m, n$ with $m=n+\mathrm{o}(n)$ )

$$
\begin{aligned}
\text { (3.33) } & -\frac{1}{2} L_{Q}^{\langle n\rangle}(z)+(n-m-1) Q^{\langle n\rangle}(z) \\
\leq & -\frac{1-\theta}{2} n(n-1) \gamma(Q)-\left[(n-1) \delta_{0} \theta-\left(1+\delta_{0}\right)(n-m-1)\right]\left(\Lambda^{\langle n\rangle}(z)-\frac{C_{0} n}{1+\delta_{0}}\right)+\frac{C_{1} \theta}{2} n(n-1) \\
= & -\frac{1-\theta}{2} n(n-1) \gamma(Q)-\left[(n-1) \delta_{0} \theta-\left(1+\delta_{0}\right)(n-m-1)\right] \Lambda^{\langle n\rangle}(z)+C_{0} \theta n(n-1)-C_{0} n(n-m-1) .
\end{aligned}
$$

We multiply by $\beta / 2$ on the left and right hand sides, to get

$$
\begin{aligned}
Z_{m, n} & =\int_{\mathbb{C}^{n}} \exp \left\{-\frac{\beta}{4} L_{Q}^{\langle n\rangle}(z)+\frac{\beta}{2}(n-m-1) Q^{\langle n\rangle}(z)\right\} \operatorname{dvol}_{2 n}(z) \\
& \leq \mathrm{e}^{-\frac{\beta}{4}(1-\theta) n(n-1) \gamma(Q)+\frac{\beta}{2} C_{0} \theta n(n-1)-C_{0} n(n-m-1)}\left\{\int_{\mathbb{C}}\left(1+|\xi|^{2}\right)^{-\frac{\beta}{2}\left[\theta \delta_{0}(n-1)-\left(1+\delta_{0}\right)(n-m-1)\right]} \operatorname{dvol}_{2}(\xi)\right\}^{n},
\end{aligned}
$$

so that in view of (3.24), we have

$$
Z_{m, n} \leq \exp \left\{-\frac{\beta}{4}(1-\theta) \gamma(Q) n(n-1)+\frac{C_{0} \beta}{2} \theta n(n-1)-C_{0} n(n-m-1)\right\},
$$

provided (3.26) is assumed. Taking logarithms, we find that

$$
\frac{1}{n(n-1)} \log Z_{m, n} \leq-\frac{\beta}{4}(1-\theta) \gamma(Q)+\frac{C_{0} \beta}{2} \theta-C_{0}\left(1-\frac{m}{n-1}\right),
$$


for big enough $m, n$ with $m=n+\mathrm{o}(n)$, since (3.26) is fulfilled then. As $\theta, 0<\theta<1$, can be taken as close to 0 as we like, it follows that

$$
\lim \sup \frac{1}{n(n-1)} \log Z_{m, n} \leq-\frac{\beta}{4} \gamma(Q) .
$$

The claim is an immediate consequence.

3.5. The proof of Johansson's marginal probability theorem. For a positive real $R$ (a radius), we put

$$
n_{R}(z)=\sharp\left\{j \in\{1, \ldots, n\}:\left|z_{j}\right| \geq R\right\},
$$

where $\sharp$ counts the number of elements, and $z=\left(z_{1}, \ldots, z_{n}\right)$, as before. We let $R_{0}$ be a positive real with

$$
\delta_{0} \log \left(1+R_{0}^{2}\right) \geq \gamma(Q)+2 C_{0}+1 .
$$

Proposition 3.2. We have the estimate

$$
\frac{n_{R_{0}}(z)}{n} \leq \epsilon, \quad z \in \mathcal{A}(n, \epsilon)
$$

Proof. We split the integer interval:

$$
\{1, \ldots, n\}=\mathfrak{n}\left(z, R_{0}\right) \cup \mathfrak{m}\left(z, R_{0}\right), \quad \mathfrak{n}\left(z, R_{0}\right) \cap \mathfrak{m}\left(z, R_{0}\right)=\emptyset,
$$

where

$$
\mathfrak{n}\left(z, R_{0}\right)=\left\{j \in\{1, \ldots, n\}:\left|z_{j}\right| \geq R\right\},
$$

so that $n_{R_{0}}(z)=\sharp\left[\mathfrak{n}\left(z, R_{0}\right)\right]$. We split the sum defining $L^{\langle n\rangle}(z)$ accordingly (we use the symmetry $\left.L_{Q}(\xi, \eta)=L_{Q}(\eta, \xi)\right)$ :

$$
\begin{aligned}
L^{\langle n\rangle\rangle}(z)=L_{Q}^{\mathrm{I}}(z)+2 L_{Q}^{\mathrm{II}}(z)+L_{Q}^{\mathrm{III}}(z) & \sum_{j, k \in \mathfrak{m}\left(z, R_{0}\right): j \neq k} L_{Q}\left(z_{j}, z_{k}\right)+2 \sum_{j \in \mathfrak{m}\left(z, R_{0}\right), k \in \mathfrak{n}\left(z, R_{0}\right)} L_{Q}\left(z_{j}, z_{k}\right)+\sum_{j, k \in \mathfrak{n}\left(z, R_{0}\right): j \neq k} L_{Q}\left(z_{j}, z_{k}\right),
\end{aligned}
$$

with the obvious interpretation of $L_{Q}^{\mathrm{I}}(z), L_{Q}^{\mathrm{II}}(z)$, and $L_{Q}^{\mathrm{III}}(z)$. From the extra growth condition (2.13), we see that

so that by $(\underline{3.35)}$,

$$
L_{Q}(\xi, \eta) \geq G(\xi, \eta)+\delta_{0} \log \left[\left(1+|\xi|^{2}\right)\left(1+|\eta|^{2}\right)\right]-2 C_{0}
$$

$$
L_{Q}(\xi, \eta) \geq \delta_{0} \log \left[\left(1+R_{0}^{2}\right)\right]-2 C_{0} \geq \gamma(Q)+1, \quad \text { if } \quad|\eta| \geq R_{0} .
$$

This allows us to conclude that

$$
2 L_{Q}^{\mathrm{II}}(z)+L_{Q}^{\mathrm{III}}(z) \geq 2 n_{R_{0}}(z)\left[n-n_{R_{0}}(z)\right](\gamma(Q)+1)+n_{R_{0}}(z)\left[n_{R_{0}}(z)-1\right](\gamma(Q)+1),
$$

As regards the term $L_{Q}^{\mathrm{I}}(z)$, we may apply (3.15) to the remaining $\left(n-n_{R_{0}}(z)\right)$-tuple:

$$
L_{Q}^{\mathrm{I}}(z) \geq\left(n-n_{R_{0}}(z)\right)\left(n-n_{R_{0}}(z)-1\right) \gamma(Q) .
$$

By adding up the terms, we find that

$$
L_{Q}^{\langle n\rangle}(z)=L_{Q}^{\mathrm{I}}(z)+2 L_{Q}^{\mathrm{II}}(z)+L_{Q}^{\mathrm{III}}(z) \geq n(n-1) \gamma(Q)+(n-1) n_{R_{0}}(z) .
$$

For $z \in \mathcal{A}(n, \epsilon)$, we then get

$$
\gamma(Q)+\frac{n_{R_{0}}(z)}{n} \leq \frac{1}{n(n-1)} L^{\langle n\rangle}(z) \leq \gamma(Q)+\epsilon,
$$

from which the assertion is immediate. 
For a point $z \in \mathbb{C}^{n}$, we define the associated weighted sum of point masses $\sigma_{z} \in \mathcal{P}_{c}(\mathbb{C})$ by the formula

$$
\mathrm{d} \sigma_{z}(\xi)=\frac{1}{n} \sum_{j=1}^{n} \mathrm{~d} \delta_{z_{j}}(\xi), \quad \xi \in \mathbb{C},
$$

where $\delta_{w}$ means the Dirac point mass at $w \in \mathbb{C}$. Also, let $C_{b}(\mathbb{C})=C(\mathbb{C}) \cap L^{\infty}(\mathbb{C})$ denote the space of bounded complex-valued continuous functions on $\mathbb{C}$.

Proposition 3.3. Suppose $\sigma_{n}=\sigma_{z}$ is as above, with $z=\left(z_{1}, \ldots, z_{n}\right) \in \mathbb{C}^{n}$. Suppose, moreover, that

$$
I_{Q}^{\sharp}\left[\sigma_{n}\right]=\frac{1}{n(n-1)} L^{\langle n\rangle}(z) \rightarrow \gamma(Q)
$$

as $n \rightarrow+\infty$. Then, as $n \rightarrow+\infty$, we have $\sigma_{n} \rightarrow \hat{\sigma}_{Q}$ weakly-star. In other words, for each $f \in C_{b}(\mathbb{C})$, we have

$$
\int_{\mathbb{C}} f \mathrm{~d} \sigma_{n} \rightarrow \int_{\mathbb{C}} f \mathrm{~d} \hat{\sigma}_{Q} \text { as } n \rightarrow+\infty .
$$

Proof. The proof is standard. We choose a weakly-star convergent subsequence, and call the limit $\sigma^{*}$. From the assumptions on the probaility measure $\sigma_{n}$, we find that almost all its mass is concentrated to a fixed compact subset of $\mathbb{C}$ (cf. Proposition 3.3), and that $I_{Q}\left[\sigma^{*}\right] \leq I_{Q}\left[\hat{\sigma}_{Q}\right]=\gamma(Q)$, by considering a cut-off of the logarithmic kernel. We leave the details to the interested reader.

Let $\omega:\{1, \ldots, n\} \rightarrow\{1, \ldots, n\}$ be a permutation. For $z=\left(z_{1}, \ldots, z_{n}\right) \in \mathbb{C}^{n}$ we let $z^{\omega}=$ $\left(z_{\omega(1)}, \ldots, z_{\omega(n)}\right) \in \mathbb{C}^{n}$ be point induced by the permutation. Suppose for the moment that $f \in C_{b}\left(\mathbb{C}^{n}\right)$, and write $f^{\omega}(z)=f\left(z^{\omega}\right)$. By symmetry, we then have

$$
\int_{\mathbb{C}^{n}} f \mathrm{~d} \Pi_{m Q, n}=\int_{\mathbb{C}^{n}} f^{\omega} \mathrm{d} \Pi_{m Q, n}
$$

which gives

$$
\int_{\mathbb{C}^{n}} f \mathrm{~d} \Pi_{m Q, n}=\frac{1}{n !} \int_{\mathbb{C}^{n}} \sum_{\omega} f^{\omega} \mathrm{d} \Pi_{m Q, n},
$$

where the sum runs over all permutations $\omega$. We next split the integral:

$$
\int_{\mathbb{C}^{n}} f \mathrm{~d} \Pi_{m Q, n}=\frac{1}{n !} \int_{\mathcal{A}(n, \epsilon)} \sum_{\omega} f^{\omega} \mathrm{d} \Pi_{m Q, n}+\frac{1}{n !} \int_{\mathbb{C}^{n} \backslash \mathcal{A}(n, \epsilon)} \sum_{\zeta} f^{\omega} \mathrm{d} \Pi_{m Q, n} .
$$

By Propositions 3.1 and 3.2 the last term is $\mathrm{o}(1)$ as $m, n \rightarrow+\infty$ while $m=n+\mathrm{o}(n)$. In order to understand the remaining term, we should study

$$
\frac{1}{n !} \sum_{\omega} f^{\omega} \quad \text { on } \mathcal{A}(n, \epsilon)
$$

We now focus on the $k=1$ case of Johansson's theorem, and restrict our attention to $f$ which only depend on the first coordinate, $f(z)=f\left(z_{1}\right)$ with some slight abuse of notation. Then (3.38) amounts to the linear statistic

$$
\frac{1}{n} \sum_{j=1}^{n} f\left(z_{j}\right), \quad\left(z_{1}, \ldots, z_{n}\right) \in \mathcal{A}(n, \epsilon) .
$$

By Proposition 3.2. only an $\epsilon$ proportion of the points $z_{j}$ may fall outside the disk $\mathbb{D}\left(0, R_{0}\right)$, and by Proposition 3.3, the expression (3.39) is close to (the constant!)

$$
\int_{\mathbb{C}} f \mathrm{~d} \hat{\sigma}_{Q}
$$

for small $\epsilon$ and large $n$. The weak-star convergence $\Pi_{m Q, n}^{(1)} \rightarrow \hat{\sigma}_{Q}$ follows, if we let $\epsilon$ approach 0 slowly as $n \rightarrow+\infty$. The remaining case $k>1$ is analogous. 


\section{An obstacle problem. Smooth potentials}

4.1. Equilibrium measure in terms of an obstacle problem. We consider the cone $\operatorname{Sub}(\mathbb{C})$ of all subharmonic functions in the plane $\mathbb{C}$, and its convex subset $(0<t<+\infty$ is assumed fixed)

$$
\operatorname{Sub}_{t}(\mathbb{C}):=\left\{v \in \operatorname{Sub}(\mathbb{C}): \limsup _{|z| \rightarrow+\infty}\left[v(z)-t \log |z|^{2}\right]<+\infty\right\} .
$$

Given $Q: \mathbb{C} \rightarrow \mathbb{R} \cup\{+\infty\}$, the obstacle problem is to find

$$
\operatorname{Obst}_{t}[Q](z):=\sup \left\{v(z): v \in \operatorname{Sub}_{t}(\mathbb{C}) \text { and } v \leq Q \text { on } \mathbb{C}\right\} \text {. }
$$

Here, we assume of $Q$ - as before - that it is lower semi-continuous, bounded on a set of positive area, and that

$$
\lim _{|z| \rightarrow+\infty}\left[Q(z)-t \log |z|^{2}\right]=+\infty
$$

We think of both $Q$ and $t$ as fixed; we observe, however, that if (4.2) is fulfilled for one value of $t$, then any smaller positive value works as well. It is easy to check that the supremum in (4.1) is taken over a non-empty collection of functions $v$ (e.g., a large negative constant will satisfy the requirements). See e.g. Doob [6] for the potential theory pertaining to obstacle problems of this type. For instance, after possibly redefining the function $\mathrm{Obst}_{t}[Q]$ on a negligible set (here, this is a set of logarithmic capacity 0 ), we get a subharmonic function. We need to connect the obstacle problem (4.1) with the equilibrium measure theory of Subsections 2.6 and 2.9. To this end, let

$$
\hat{\sigma}_{t}=\hat{\sigma}_{t}[Q]:=t \hat{\sigma}_{Q / t}, \quad S_{t}=S_{t}[Q]:=S_{Q / t}=\operatorname{supp} \hat{\sigma}_{t}
$$

be the scaled equilibrium measure of Subsection 2.9 and its associated support set. We write

$$
\gamma_{t}(Q)=t \gamma(Q / t) \quad \text { and } \quad \gamma_{t}^{*}(Q)=\gamma_{t}(Q)-\frac{1}{t} \int_{\mathbb{C}} Q \mathrm{~d} \hat{\sigma}_{t} .
$$

For a compactly supported finite positive Borel measure $\sigma$, let $U^{\sigma}$ denote the logarithmic potential

$$
U^{\sigma}(\xi)=\int_{\mathbb{C}} \log \frac{1}{|\xi-\eta|^{2}} \mathrm{~d} \sigma(\eta)
$$

and put

$$
\widehat{Q}_{t}(\xi)=\gamma_{t}^{*}(Q)-U^{\hat{\sigma}_{t}}(\xi) .
$$

The function $\widehat{Q}_{t}$ is then subharmonic in $\mathbb{C}$, and harmonic in $\mathbb{C} \backslash S_{t}$, where $S_{t}=$ supp $\hat{\sigma}_{t}$. Moreover, as it is the total mass of the measure which determines the decay of the logarithmic potential at infinity, we have

$$
\widehat{Q}_{t}(z)=t \log |z|^{2}+\mathrm{O}(1) \quad \text { as }|z| \rightarrow+\infty .
$$

The following lemma supplies a criterion which allows us to solve the obstacle problem. We recall that the logarithmic energy $I_{0}[\sigma]$ is given by $(2.8)$ with $Q$ replaced by 0 .

Lemma 4.1. Let $\sigma$ be a compactly supported finite positive Borel measure in $\mathbb{C}$ of finite logarithmic energy $I_{0}[\sigma]<+\infty$ with total mass $\|\sigma\|=t$. Suppose $W=c-U^{\sigma}$, where $c \in \mathbb{R}$ is a constant. If $W$ has both $W \leq Q$ q.e. on $\mathbb{C}$ and $W=Q$ q.e. on $\operatorname{supp} \sigma$, then $W=\operatorname{Obst}_{t}[Q]$ q.e.

Proof. Without loss of generality, we may assume that $Q \geq 1$ on $\mathbb{C}$. The function $W$ is in $\operatorname{Sub}_{t}(\mathbb{C})$ while $W \leq Q$ q.e. on $\mathbb{C}$. So $W \leq Q$ on $\mathbb{C} \backslash E$, where $E \subset \mathbb{C}$, is polar (i.e., has logarithmic capacity $0)$. Let $\rho$ be a compactly supported Borel measure on $\mathbb{C}$ such that the corresponding potential has $U^{\rho}=+\infty$ on $E$ (see, e.g. [6]). Put $W^{\prime}:=(1-\epsilon) W-\epsilon^{\prime} U^{\rho}$ where $\epsilon, \epsilon^{\prime}$ are two small positive numbers. If $\epsilon^{\prime}$ is very small (also relative to $\epsilon$ ), we can make sure that $W^{\prime} \leq Q$ on $\mathbb{C} \backslash E$, by using that $W \leq Q$ on $\mathbb{C} \backslash E$, the standard properties of potentials, and the given properties of $Q$. Then $W^{\prime} \leq Q$ throughout $\mathbb{C}$ automatically as $W^{\prime}=-\infty$ on $E$. We conclude that $W^{\prime} \leq \operatorname{Obst}_{t}[Q]$ on $\mathbb{C}$. By 
letting $\epsilon^{\prime} \rightarrow 0$ first and then $\epsilon \rightarrow 0$ second, we get that $W \leq \operatorname{Obst}_{t}[Q]$ on $\mathbb{C} \backslash E$, and consequently, $W \leq$ Obst $_{t}[Q]$ q.e. on $\mathbb{C}$. As a side remark, we observe that $W$ is locally of Sobolev class $W^{1,2}$, which means that in the sense of distributions, its gradient is locally in $L^{2}$ with respect to area measure. It remains to show the reverse inequality $\operatorname{Obst}_{t}[Q] \leq W$ q.e. on $\mathbb{C}$. To this end, we pick a function $v \in \operatorname{Sub}_{t}(\mathbb{C})$ with $v \leq Q$ q.e. on $\mathbb{C}$. It will suffice to show that $v \leq W$ on $\mathbb{C}$. The potential $U^{\sigma}$ is harmonic in $\mathbb{C} \backslash S$, where $S:=\operatorname{supp} \sigma$, and therefore $W$ is harmonic there as well. The assumption on the total mass of $\sigma$ gives that

$$
W(z)=t \log |z|^{2}+\mathrm{O}(1) \quad \text { as }|z| \rightarrow+\infty .
$$

Next, we consider the difference $u=v-W$, which is subharmonic in $\mathbb{C} \backslash S$ and has $u \leq 0$ q.e. on $S$. Moreover, the assumption that $v \in \operatorname{Sub}_{t}(\mathbb{C})$ together with (4.5) shows that $u$ is bounded from above near infinity. We should like to apply the maximum principle in the open set $\mathbb{C} \backslash S$ and obtain that $u \leq 0$ on $\mathbb{C} \backslash S$ since $u \leq 0$ q.e. on the boundary. However, this is a little delicate as the functions are not necessarily continuous up to the boundary. The so-called Principle of Domination [20], p. 104, is a good substitute. To apply it, we need to make the technical assumption that $v$ is harmonic in a punctured neighborhood of infinity, because it allows us to represent $v$ q.e. in the form $b-U^{v}$, where $b$ is a constant and $v$ is finite compactly supported positive Borel measure. The assumption $v \in \operatorname{Sub}_{t}(\mathbb{C})$ then gives that $v$ has total mass $\|v\| \leq t$, so that $\|v\| \leq\|\sigma\|$. From the assumptions we read off that $U^{\sigma} \leq U^{v}+c-b$ holds q.e. on $S=\operatorname{supp} \sigma$, and hence $\sigma$-a.e. (because $\sigma$ has finite logarithmic energy), so by the Principle of Domination (which again uses that $\sigma$ has finite logarithmic energy, and that $\|v\| \leq\|\sigma\|$ ), we find that $U^{\sigma} \leq U^{v}+c-b$ holds throughout $\mathbb{C}$. The desired conclusion that $v \leq W$ follows. Next, to justify the conclusion that $v \leq W$ on $\mathbb{C}$ holds when we only assume that $v \in \operatorname{Sub}_{t}(\mathbb{C})$ with $v \leq Q$ q.e. on $\mathbb{C}$, we proceed as follows. If we let $\varepsilon$ be a small positive real number, and put

$$
\tilde{v}(z):=\max \left\{\frac{v(z)}{1+\varepsilon}, t \log |z|^{2}-C\right\},
$$

then $\tilde{v} \in \operatorname{Sub}_{t}(\mathbb{C})$ is harmonic in a punctured neighborhood of the point at infinity, and $\tilde{v} \leq Q$ q.e. on $\mathbb{C}$ holds if the constant $C$ is big enough positive. So we have the conclusion $\tilde{v} \leq W$ on $\mathbb{C}$ from the previous argument. Finally, we first let $C \rightarrow+\infty$ and afterwards let $\varepsilon \rightarrow 0$, and obtain $v \leq W$, as claimed.

We have the following characterization.

Proposition 4.2. We have

$$
\operatorname{Obst}_{t}[Q](z)=\widehat{Q}_{t}(z), \quad \text { q.e. }-z \in \mathbb{C} \text {. }
$$

Proof. In view of Lemma 4.1, we just need to check that $\widehat{Q}_{t}=Q$ q.e. on $S_{t}$ while $\widehat{Q}_{t} \leq Q$ q.e. on $\mathbb{C}$. By Frostman's Theorem 2.6, we have $U^{\hat{Q}_{Q / t}}+Q / t \geq \gamma^{*}(Q / t)$ q.e. on $\mathbb{C}$, while $U^{\hat{o}_{Q / t}}+Q / t=\gamma^{*}(Q / t)$ q.e. on $S_{Q / t}$. Since $\hat{\sigma}_{t}=t \hat{\sigma}_{Q / t}, S_{t}=S_{Q / t}$, and $\gamma_{t}^{*}(Q)=t \gamma^{*}(Q / t)$, this means that $U^{\hat{\sigma}_{t}}+Q \geq \gamma_{t}^{*}(Q)$ q.e. on $\mathbb{C}$, while $U^{\hat{\sigma}_{t}}+Q=\gamma_{t}^{*}(Q)$ q.e. on $S_{t}$. With $\widehat{Q}_{t}=\gamma_{t}^{*}(Q)-U^{\hat{\sigma}_{t}}$, this is the same as having $\widehat{Q}_{t} \leq Q$ q.e. on $\mathbb{C}$, while $\widehat{Q}_{t}=Q$ q.e. on $S_{t}$, as needed.

Remark 4.3. The assertion of Proposition 4.2] is essentially equivalent to that of Theorem I.4.1 [20].

We easily recover the density from the potential; we write $\mathrm{d} A:=\pi^{-1} \mathrm{dvol}_{2}$ for normalized area measure.

Corollary 4.4. We have, in the sense of distribution theory, $\mathrm{d} \hat{\sigma}_{t}=\Delta \widehat{Q}_{t} \mathrm{~d} A$. 
4.2. The super-coincidence and coincidence sets. We keep the setting of the previous subsection, and assume $Q: \mathbb{C} \rightarrow \mathbb{R} \cup\{+\infty\}$ is lower semi-continuous, and bounded on a set of positive area, subject to the growth condition (4.2). The potential $U^{\sigma}$ is superharmonic for a given finite positive compactly supported measure $\sigma$, and therefore the function $\widehat{Q}_{t}$ defined by (4.4) is automatically subharmonic. In particular, $\widehat{Q}_{t}$ is upper semi-continuous, and we find that the difference $Q-\widehat{Q}_{t}$ is lower semi-continuous. It follows that the super-coincidence set

$$
S_{t}^{*}=S_{t}^{*}[Q]=\left\{z \in \mathbb{C}: \widehat{Q}_{t}(z) \geq Q(z)\right\}
$$

is compact: it is closed by semi-continuity, while (4.4) and (4.5) show that it is bounded. We note that by Proposition $4.2, \widehat{Q}_{t} \leq Q$ quasi-everywhere, so that $S_{t}^{*}$ equals, up to a set of logarithmic capacity zero, the coincidence set

$$
\left\{z \in \mathbb{C}: \widehat{Q}_{t}(z)=Q(z)\right\} .
$$

In all cases when we have a little regularity, $\widehat{Q}_{t}$ is continuous, and then the super-coincidence set $S_{t}^{*}$ is the same as the coincidence set. We therefore refrain from introducing separate notation for the coincidence set.

Proposition 4.5. The function $\widehat{Q}_{t}$ is harmonic in $\mathbb{C} \backslash S_{t}^{*}$, and as a consequence, $S_{t} \subset S_{t}^{*}$. In particular, $S_{t}^{*}$ is non-empty.

Proof. We pick a point $z_{0} \in \mathbb{C} \backslash S_{t}^{*}$, so that $\widehat{Q}_{t}\left(z_{0}\right)<Q\left(z_{0}\right)$. By semi-continuity, we get that $\widehat{Q}_{t}<Q$ in a neighborhood of $z_{0}$. We claim that $\widehat{Q}_{t}$ is harmonic near $z_{0}$. If not, we could use Perron's lemma and replace $\mathrm{Obst}_{t}[Q]$ (which equals $\widehat{Q}_{t}$ q.e., by Proposition 4.2) on a small disk around $z_{0}$ by the harmonic function which has the same boundary values, and get a function which is in $\operatorname{Sub}_{t}(\mathbb{C})$, and bigger than Obst $_{t}[Q]$ while being $\leq Q$. This violates the extremality of Obst $_{t}[Q]$, and the claim follows. Next, by Corollary 4.4, we see that $z_{0} \in \mathbb{C} \backslash S_{t}$. Since $z_{0}$ was an arbitrary point in $\mathbb{C} \backslash S_{t}^{*}$, the proof is complete.

4.3. A priori smoothness for the obstacle problem for smooth potentials. As before, $Q: \mathbb{C} \rightarrow$ $\mathbb{R} \cup\{+\infty\}$ is lower semi-continuous with (4.2) where $t$ is a (fixed) positive real. If $Q$ has some degree of smoothness, say, e.g., $Q: \mathbb{C} \rightarrow \mathbb{R}$ is $C^{2}$-smooth, it is natural to wonder to what extent that carries over to $\mathrm{Obst}_{t}[Q]=\widehat{Q}_{t}$. Since we sometimes need to work with slightly less smooth weights, the (local) Sobolev classes $W^{2, p}$ are sometimes more appropriate. By Sobolev imbedding, functions in $W^{2, p}$ are continuous provided $1<p<+\infty$. The case $p=+\infty$ gives the space $W^{2, \infty}=C^{1,1}$ of functions whose first order partial derivatives are locally Lipschitz continuous. We use the notation $W^{2, p}$ and $C^{1,1}$ for local classes unless otherwise stated.

The following a priori smoothness result is standard in connection with the constrained obstacle problem discussed below [9], and associated with the names such as Lewy, Stampacchia, Brezis, Lions, Kinderlehrer, and Caffarelli. We present the elementary approach recently found by Berman [4], which gives the $C^{1,1}$-smoothness part. Berman's approach also applies in the several complex variables context.

Proposition 4.6. If $Q \in C^{2}$, then $\operatorname{Obst}_{t}[Q] \in C^{1,1}$. More generally, if $Q \in C^{1,1}$, we still have $\operatorname{Obst}_{t}[Q] \in$ $C^{1,1}$. Finally, if $Q \in W^{2, p}$ for some $p, 1<p<+\infty$, then $\operatorname{Obst}_{t}[Q] \in W^{2, p}$.

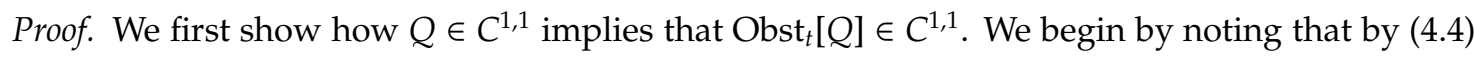
and Proposition 4.2 .

$$
\operatorname{Obst}_{t}[Q](z)=\widehat{Q}_{t}(z) \leq t \log \left(1+|z|^{2}\right)+C, \quad z \in \mathbb{C},
$$

for a suitable real constant $C$. By (4.2), the growth of $Q(z)$ is faster than that of Obst $_{t}[Q]$, which we can use to show that for some possibly big value of the radius $r_{0}$,

$$
\text { Obst }_{t}[Q](z+w) \leq Q(z), \quad|z| \geq r_{0},|w| \leq 1 .
$$


Let

$$
M_{r}:=\sup \left\{\left|\partial_{t}^{2}\{Q(z+t \zeta)\}\right|:|z| \leq r,|\zeta|=1,0 \leq t \leq 1\right\},
$$

which is finite for each radius $r$ due to the assumption that $Q \in C^{1,1}$, and note that by Taylor's formula,

$$
\operatorname{Obst}_{t}[Q](z+w) \leq Q(z+w) \leq Q(z)+2 \operatorname{Re}[w \partial Q(z)]+\frac{1}{2} M_{r}|w|^{2}, \quad|z| \leq r,|w| \leq 1 .
$$

We fix $w \in \mathbb{C}$ with $|w| \leq 1$, and put

$$
\tilde{Q}_{w}(z):=\frac{1}{2} \operatorname{Obst}_{t}[Q](z+w)+\frac{1}{2} \operatorname{Obst}_{t}[Q](z-w)-\frac{1}{2} M_{r_{0}}|w|^{2} .
$$

By a combination of (4.7) and (4.8), $\tilde{Q}_{w} \leq Q$ on $\mathbb{C}$, while it is obvious that $\tilde{Q}_{w} \in \operatorname{Sub}_{t}(\mathbb{C})$. So, from the definition of the obstacle problem, we see that $\tilde{Q}_{w} \leq \operatorname{Obst}_{t}[Q]$ on $\mathbb{C}$. In other words,

$$
\operatorname{Obst}_{t}[Q](z+w)+\operatorname{Obst}_{t}[Q](z-w)-2 \operatorname{Obst}_{t}[Q](z) \leq M_{r_{0}}|w|^{2}, \quad z \in \mathbb{C},|w| \leq 1 .
$$

Next, if we divide both sides of (4.9) by $|w|^{2}$ and then let $w \rightarrow 0$, we get

$$
\left.\partial_{t}^{2} \mathrm{Obst}_{t}[Q](z+t \zeta)\right|_{t=0} \leq M_{r_{0}}, \quad z \in \mathbb{C},|\zeta|=1 .
$$

In particular, if $z=x+\mathrm{i} y$, we have

$$
\partial_{x}^{2} \operatorname{Obst}_{t}[Q](z) \leq M_{r_{0}}, \quad \partial_{y}^{2} \operatorname{Obst}_{t}[Q](z) \leq M_{r_{0}} .
$$

Since $\mathrm{Obst}_{t}[Q]$ is subharmonic, that is,

$$
\partial_{x}^{2} \mathrm{Obst}_{t}[Q](z)+\partial_{y}^{2} \mathrm{Obst}_{t}[Q](z) \geq 0 .
$$

holds in the sense of distribution theory, we must then also have

$$
-M_{r_{0}} \leq \partial_{x}^{2} \mathrm{Obst}_{t}[Q](z) \leq M_{r_{0}}, \quad-M_{r_{0}} \leq \partial_{y}^{2} \operatorname{Obst}_{t}[Q](z) \leq M_{r_{0}} .
$$

In particular, then, $\mathrm{Obst}_{t}[Q] \in C^{1,1}$.

As for the remaining case when we have less smoothness, that is, when $Q \in W^{2, p}$, the assertion follows from the smoothness theory of constrained obstacle problems (see Lemma 4.7 and Theorem 4.9 below).

4.4. A constrained obstacle problem. Let $Q: \mathbb{C} \rightarrow \mathbb{R} \cup\{+\infty\}$ be lower semi-continuous with (4.2) where $t$ is a (fixed) positive real, as before. Let $\Omega$ be a (bounded) Jordan domain, and $\varrho: \partial \Omega \rightarrow \mathbb{R}$ a continuous function with $\varrho \leq\left. Q\right|_{\partial \Omega}$. Consider the constrained obstacle problem

$$
\text { Obst }_{\Omega, \varrho}[Q](z):=\sup \{v(z): v \in \operatorname{Sub}(\Omega), v \leq Q \text { on } \Omega, v=\varrho \text { on } \partial \Omega\}, \quad z \in \bar{\Omega} .
$$

We would like to model the obstacle problem associated with $\mathrm{Obst}_{t}[Q]$ in the form of such a constrained obstacle problem. The natural way to do this is to put $\varrho:=\left.\operatorname{Obst}_{t}[Q]\right|_{\partial \Omega}$.

Lemma 4.7. If $\Omega$ is a $C^{\infty}$-smooth bounded Jordan domain and $\varrho=\left.\operatorname{Obst}_{t}[Q]\right|_{\partial \Omega^{\prime}}$ then

$$
\operatorname{Obst}_{\Omega, \varrho}[Q]=\operatorname{Obst}_{t}[Q] \text { on } \Omega \text {. }
$$

Proof. We put $R_{0}=\operatorname{Obst}_{t}[Q], R_{1}=\left.\operatorname{Obst}_{t}[Q]\right|_{\Omega^{\prime}}$ and $R_{2}=$ Obst $_{\Omega, \varrho}[Q]$. The function $R_{1}$ is subharmonic with $R_{1} \leq Q$ in $\Omega$, and has boundary values $\left.R_{1}\right|_{\partial \Omega}=\varrho$. It is now immediate that $R_{1} \leq R_{2}$. We proceed to show that $R_{2} \leq R_{1}$. To this end, we let $v \in \operatorname{Sub}(\Omega)$ have $v \leq Q$ on $\Omega$ and boundary data $v=\varrho$ on $\partial \Omega$; we are to check that $v \leq R_{1}$. Next, we put $\tilde{v}=\max \left\{v, R_{1}\right\}$; the function $\tilde{v}$ is in $\operatorname{Sub}(\Omega)$, has $R_{1} \leq \tilde{v} \leq Q$ on $\Omega$, and boundary data $\left.\tilde{v}\right|_{\partial \Omega}=\varrho$. We consider its extension

$$
V=\left\{\begin{array}{l}
\tilde{v} \quad \text { in } \Omega, \\
R_{0} \quad \text { in } \mathbb{C} \backslash \Omega .
\end{array}\right.
$$


The way things are set up, $R_{0} \leq V \leq Q$ in $\mathbb{C}$, with $V=R_{0}$ on $\partial \Omega$. We claim that $V \in \operatorname{Sub}(\mathbb{C})$. It is enough to check the mean value inequality along $\partial \Omega$. For points $a \in \partial \Omega$, we have $(\epsilon>0$ is a small real parameter)

$$
V(a)=R_{0}(a) \leq \frac{1}{2 \pi} \int_{-\pi}^{\pi} R_{0}\left(a+\epsilon \mathrm{e}^{\mathrm{i} \theta}\right) \mathrm{d} \theta \leq \int_{-\pi}^{\pi} V\left(a+\epsilon \mathrm{e}^{\mathrm{i} \theta}\right) \mathrm{d} \theta .
$$

It follows that $V \in \operatorname{Sub}(\mathbb{C})$ and a fortiori $V \in \operatorname{Sub}_{t}(\mathbb{C})$ (because of the growth at infinity). We conclude that $V$ is a function which we may plug into the optimization problem defining $R_{0}=$ Obst $_{t}[Q]$, and so $V \leq R_{0}$ on $\mathbb{C}$. In fact, due to the reverse inequality, we must have $V=R_{0}$. In particular, $\tilde{v}=\left.V\right|_{\bar{\Omega}}=R_{1}$, and so $v \leq R_{1}$.

4.5. Kinderlehrer-Stampacchia-Caffarelli theory. For our purposes it would be enough to consider the case of $C^{2}$ or even $C^{\infty}$ potentials $Q$ but since we sometimes have to modify them (see e.g. [2]), the Sobolev classes $W^{2, p}$ seem to be more appropriate. We generally assume that $Q: \mathbb{C} \rightarrow \mathbb{R}$ is continuous subject to the growth condition (4.2) for some (fixed) positive real $t$.

We start with a simple observation.

Lemma 4.8. Let $S_{t}=S_{t}[Q]$ and suppose $Q \in W^{2,1}\left(\operatorname{int} S_{t}\right)$. Then $\hat{\sigma}_{t}$ is absolutely continuous in int $S_{t}$ and in fact

$$
\mathrm{d} \hat{\sigma}_{t}=\Delta Q \mathrm{~d} A \quad \text { on } \operatorname{int} S_{t} .
$$

Proof. As we know that $\mathrm{d} \sigma_{t}=\Delta \widehat{Q}_{t} \mathrm{~d} A$ in the sense of distributions, and so the same is true if we restrict the distributions to the open set int $S_{t}$, where $\widehat{Q}_{t}=Q$, and therefore $\Delta \widehat{Q}_{t}=\Delta Q$ as distributions.

The following two theorems are adapted from the theory of constrained obstacle problems (variational inequalities); this theory is, as mentioned previously, associated with the names of Lewy, Stampacchia, Brezis, Lions, Kinderlehrer, and Caffarelli, et al. A standard references is [9], Chapter 1 (see also [5]).

Theorem 4.9. Fix $p, 1<p<+\infty$, and let $\Omega$ be a $C^{\infty}$-smooth bounded Jordan domain. We suppose $Q$ is $W^{2, p}$-smooth in $\mathbb{C}$, and that $\varrho: \partial \Omega \rightarrow \mathbb{R}$ is a function which is the restriction to $\partial \Omega$ of a function in $W^{2, p}(\mathbb{C})$, with $\varrho \leq Q$ on $\partial \Omega$. Then $\operatorname{Obst}_{\Omega, \varrho}[Q] \in W^{2, p}(\Omega)$.

Proof. This is explained in Chapter 1 of Friedman's book [9], see Theorem 1.3.2 and Problem 1 on p. 29.

Together with Lemma4.7 this justifies the $W^{2, p}$ part of the assertion of Proposition 4.6 Now, in view of Proposition 4.6, if we suppose $Q \in W^{2, p}$ for some $1<p<+\infty$, the function $\widehat{Q}_{t}=$ Obst $_{t}[Q]$ is in $W^{2, p}$, and by Corollary 4.4, the measure $\hat{\sigma}_{t}$ is absolutely continuous (with respect to area), and the density is locally in $L^{p}$. By Lemma 4.8 , we get

$$
\mathrm{d} \hat{\sigma}_{t}=\Delta Q \mathrm{~d} A \quad \text { on } \operatorname{int} S_{t} .
$$

If the boundary $\partial S_{t}$ has zero area, we can conclude that $\mathrm{d} \hat{\sigma}_{t}=1_{S_{t}} \Delta Q \mathrm{~d} A$. It is remarkable that this conclusion holds even when $\partial S_{t}$ has positive area.

Theorem 4.10. If, for some $1<p<+\infty$, we have $Q \in W^{2, p}$, then $\widehat{Q}_{t} \in W^{2, p}$ and

$$
\mathrm{d} \hat{\sigma}_{t}=1_{S_{t}} \Delta Q \mathrm{~d} A .
$$


Proof. As $S_{t}$ is the support of $\hat{\sigma}_{t}$, the measure $\hat{\sigma}_{t}$ vanishes off $S_{t}$. On $S_{t}$, however, the two $W^{2, p_{-}}$ smooth functions $Q$ and $\widehat{Q}_{t}$ coincide, and by [18], p. 53, this entails that their partial derivatives of order $\leq 2$ coincide almost everywhere on $S_{t}$. In particular, $\Delta \widehat{Q}_{t}=\Delta Q$ on $S_{t}$ as $L^{p}$ functions. In view of Corollary 4.4 , the assertion is immediate.

Remark 4.11. (a) In the context of Proposition 4.6, it does not help to add more smoothness to $Q$. E.g., if $Q \in C^{\infty}$ is assumed, we still cannot do better than $\operatorname{Obst}_{t}[Q] \in C^{1,1}$, at least near $\partial S_{t}$.

(b) The smoothness assumptions of this subsection are excessive, in the sense that it suffices to have the required smoothness of $Q$ in a neighborhood of the droplet $S_{t}$. All the statements are valid under this weaker assumption.

(c) By the properties of the 2D Hilbert transform, the assertion that $\widehat{Q}_{t} \in W^{2, p}$ is equivalent to the property that the density of the absolutely continuous measure $\mathrm{d} \hat{\sigma}_{t}$ is in $L^{p}$ (locally).

4.6. The coincidence set and shallow points. As in the previous subsection, $Q: \mathbb{C} \rightarrow \mathbb{R}$ is assumed to be of (local) Sobolev class $W^{2, p}$, with $1<p<+\infty$. We assume that $Q$ meets the growth assumption (4.2) for all $t$ with $0<t<T$, where $T=T(Q)$ has $0<T \leq+\infty$.

We recall that we introduced the parameter $t$ to consider the evolution of the renormalized equilibrium measures $\hat{\sigma}_{t}=t \hat{\sigma}_{Q / t}$ as $t$ moves. The conclusion of Theorem 4.10 allows us to reduce the complexity and just study the evolution of the droplets $S_{t}=S_{t}[Q]=S_{Q / t}$. The supercoincidence set $S_{t}^{*}=S_{t}^{*}[Q]$ defined by (4.6) will be referred to as the coincidence set, because the smoothness of $Q$ makes $\widehat{Q}_{t}$ continuous.

We should explain the relationship between the sets $S_{t}$ and $S_{t}^{*}$ (we already know that $S_{t} \subset S_{t}^{*}$ ). To this end, we say that a point $z_{0} \in S_{t}^{*}$ is $Q$-shallow (with respect to $S_{t}^{*}$ ) if there exists an open disk $D$ centered at $z_{0}$ such that

$$
\int_{S_{t}^{*} \cap D}|\Delta Q| \mathrm{d} A=0
$$

The $Q$-shallow point in $S_{t}^{*}$ form a relatively open subset. We mention in passing that it follows from Theorem 4.10 that $\Delta Q \geq 0$ a.e. on $S_{t}$.

Proposition 4.12. The set $S_{t}$ is obtained from $S_{t}^{*}$ by removal of all the $Q$-shallow points.

Proof. Since $\widehat{Q}_{t}$ and $Q$ are both in $C^{1,1}$ and coincide on $S_{t}^{*}$, we get from [18], p. 53, that $\Delta \widehat{Q}_{t}=\Delta Q$ holds a.e. on $S_{t}^{*}$, so that (in the same way as Lemma 4.8 was obtained)

$$
\mathrm{d} \hat{\sigma}_{t}=1_{S_{t}^{*}} \Delta Q \mathrm{~d} A \text {. }
$$

By comparing with Lemma 4.8, we see that $\Delta Q=0$ a.e. on $S_{t}^{*} \backslash S_{t}$. To calculate the support of $\hat{\sigma}_{t}$, we must remove all the points of $S_{t}^{*}$ where there is no $|\Delta Q| \mathrm{d} A$-mass nearby, that is, the $Q$-shallow points.

4.7. Coincidence sets and the dynamics of droplets. As in the previous subsection, $Q: \mathbb{C} \rightarrow \mathbb{R}$ is assumed to be of class $W^{2, p}$, with $1<p<+\infty$. We assume that $Q$ meets the growth assumption (4.2) for all $t$ with $0<t<T$, where $T=T(Q)$ has $0<T \leq+\infty$.

The coincidence set $S_{t}^{*}=S_{t}[Q]$ defined by (4.6) is just a little bigger than $S_{t}$ (we remove the $Q$-shallow points), but it contains essential information which helps us understand the evolution of $S_{t}$ as $t$ grows.

We begin with some elementary properties.

Lemma 4.13. If $0<t_{1} \leq t_{2}<T$, then $\widehat{Q}_{t_{1}} \leq \widehat{Q}_{t_{2}}$ and, in particular, $S_{t_{1}}^{*} \subset S_{t_{2}}^{*}$.

Proof. Since $\operatorname{Sub}_{t_{1}}(\mathbb{C}) \subset \operatorname{Sub}_{t_{2}}(\mathbb{C})$, we clearly have $\operatorname{Obst}_{t_{1}}[Q] \leq \operatorname{Obst}_{t_{2}}[Q]$, from the definition of the obstacle problem. The first assertion, $\widehat{Q}_{t_{1}} \leq \widehat{Q}_{t_{2}}$, now follows from Proposition 4.2. The second assertion, the inclusion $S_{t_{1}}^{*} \subset S_{t_{2}}^{*}$, is an easy consequence of the first assertion.

Let $S_{0}^{*}$ denote the (nonempty compact) set where the global minimum of $Q$ is attained. 
Proposition 4.14. We have $S_{0}^{*} \subset S_{t}^{*}$ for all $0<t<T$.

Proof. Pick a point $a \in S_{0}^{*}$, and observe that the function $\max \left\{Q(a), \widehat{Q}_{t}\right\}$ is subharmonic (in fact, in $\left.\operatorname{Sub}_{t}(\mathbb{C})\right)$ and therefore competes with $\widehat{Q}_{t}$ for the obstacle problem. We conclude that $Q(a) \leq \widehat{Q}_{t}$. As $\widehat{Q}_{t} \leq Q$, it follows that $\widehat{Q}_{t}(a)=Q(a)$, so that $a \in S_{t}^{*}$. The proof is complete.

Proposition 4.15. If $0<t_{1} \leq t_{2}<T$, we have $S_{t_{1}} \subset S_{t_{2}}$ and $\hat{\sigma}_{t_{1}} \leq \hat{\sigma}_{t_{2}}$.

Proof. If a point $a \in S_{t_{1}}^{*} \cap S_{t_{2}}^{*}$ is $Q$-shallow with respect to $S_{t_{2}}^{*}$, then it is also $Q$-shallow with respect to $S_{t_{1}}^{*}$, since $S_{t_{1}}^{*} \subset S_{t_{2}}^{*}$, by Lemma 4.13. The first assertion, $S_{t_{1}} \subset S_{t_{2}}$, now follows from a second application of $S_{t_{1}}^{*} \subset S_{t_{2}}^{*}$. The second assertion, $\hat{\sigma}_{t_{1}} \leq \hat{\sigma}_{t_{2}}$, is a consequence of the first assertion combined with Theorem 4.10 .

Remark 4.16. This supplies the potential theoretical proof of Corollary 2.13 alluded to in Remark 2.14 .

We need the following two lemmas from [20], pp. 227-228.

Lemma 4.17. $\left(0<t_{0}<T\right)$ The map $t \mapsto S_{t}$ is monotonically increasing and left-continuous in the Hausdorff metric:

$$
S_{t} \nearrow S_{t_{0}} \text { as } t \nearrow t_{0} \text {. }
$$

This means that $S_{t_{0}}$ is in a small neighborhood of $S_{t}$ for $t<t_{0}$ close to $t_{0}$ or, equivalently, that

$$
S_{t_{0}}=\operatorname{clos} \bigcup_{t<t_{0}} S_{t}
$$

Lemma 4.18. $\left(0<t_{0}<T\right)$ We have

$$
\bigcap_{t_{0}<t<T} S_{t} \subset S_{t_{0}}^{*}
$$

In particular, if $S_{t_{0}}=S_{t_{0}}^{*}$, then

$$
S_{t_{0}}=\bigcap_{t>t_{0}} S_{t}
$$

It is easy to construct examples which show how $S_{t_{0}}^{*}$ may contain "seed points" outside the main body of $S_{t_{0}}$ which grow into (small) components of $S_{t}$ for $t>t_{0}$. The next lemma gives a criterion which guarantees that this phenomenon takes place. For a compact set $E \subset \mathbb{C}$, let phull $(E)$ denote its polynomially convex hull, that is,

$$
\text { phull(E) }:=\left\{z \in \mathbb{C}:|p(z)| \leq \max _{E}|p| \text { for all polynomials } p\right\} \text {. }
$$

The (compact) set phull( $E)$ adds to $E$ all the points of $\mathbb{C} \backslash E$ which belong to bounded connectivity components of $\mathbb{C} \backslash E$ (i.e., points invisible to Brownian motion in $\mathbb{C} \backslash E$ starting at $\infty$ ).

Lemma 4.19. For all $t_{0}, t$ with $0<t_{0}<t<T$, we have the inclusion

$$
\partial\left[\operatorname{phull}\left(S_{t_{0}}^{*}\right)\right] \subset S_{t} .
$$

Proof. The standard geometric interpretation of the polynomially convex hull gives that

$$
\partial\left[\operatorname{phull}\left(S_{t_{0}}^{*}\right)\right] \subset \partial S_{t_{0}}^{*} \subset S_{t_{0}}^{*} .
$$

We need to show that $\partial\left[\right.$ phull $\left.\left(S_{t_{0}}^{*}\right)\right] \subset S_{t}$ for all $t, t_{0}<t<T$. We argue by contradiction, and suppose that there exists a point $a \in \partial\left[\right.$ phull $\left.\left(S_{t_{0}}^{*}\right)\right]$ such that $a \in \mathbb{C} \backslash S_{t_{1}}$ for some $t_{1}$, with $t_{0}<t_{1}<T$. Then $a \in \mathbb{C} \backslash S_{t}$ for all $t$ with $t_{0}<t \leq t_{1}$, and if $t>t_{0}$ is sufficiently close to $t_{0}$, the point $a$ belongs to the unbounded component of $\mathbb{C} \backslash S_{t}$. Indeed, choose a small open neighborhood $U$ of $a$ avoiding $S_{t_{1}}$ and since, by assumption, $a \in \partial\left[\right.$ phull $\left.\left(S_{t_{0}}^{*}\right)\right]$, we may assured that there exists a point 
$b \in U \backslash$ phull $\left(S_{t_{0}}^{*}\right)$. The point $b$ belongs to the unbounded component of $\mathbb{C} \backslash S_{t_{0}}^{*}$, so we may connect $b$ with $\infty$ by a curve $\gamma$ in $\mathbb{C} \backslash S_{t_{0}}^{*} ; \gamma$ is at a positive distance from $S_{t_{0}}^{*}$. By Lemma $4.18, \gamma \subset \mathbb{C} \backslash S_{t}$ for all $t>t_{0}$ close to $t_{0}$, and so the point $b$ - and a fortiori $a$ - is in the unbounded component of $\mathbb{C} \backslash S_{t}$. Next, we consider (for $t$ with $t>t_{0}$ close to $t_{0}$ ) the function $u=\widehat{Q}_{t_{0}}-\widehat{Q}_{t}$. Then, by Lemma 4.13 , we have $u \leq 0$. Moreover, since $a \in S_{t_{0}}^{*} \subset S_{t^{*}}^{*}$, we have $\widehat{Q}_{t_{0}}(a)=\widehat{Q}_{t}(a)=Q(a)$, and therefore, $u(a)=0$. The function $\widehat{Q}_{t_{0}}$ is harmonic in $\mathbb{C} \backslash S_{t_{0}}$, and, likewise, $\widehat{Q}_{t}$ is harmonic in $\mathbb{C} \backslash S_{t}$, so we conclude that $u$ is harmonic in $\mathbb{C} \backslash S_{t}$. The function $u$ then has a local maximum at the interior point $a$, so by the strong maximum principle, we get that $u=0$ throughout $\mathbb{C} \backslash \operatorname{phull}\left(S_{t}\right)$. This does not agree with the known asymptotics (4.4). We conclude that the initial assumption must be false, so that $a \in S_{t_{1}}$ for all $t_{1}$ with $t_{0}<t_{1}<T$.

Remark 4.20. The above assertions extend to the case $t_{0}=0$ if as before $S_{0}^{*}$ is the set where the global minimum of $Q$ is attained, and we put $S_{0}=\emptyset$.

4.8. Subharmonic potentials. As before, $Q: \mathbb{C} \rightarrow \mathbb{R}$ is assumed to be of class $W^{2, p}$, so that e.g. $\Delta Q \in L_{\text {loc }}^{p}(\mathbb{C})$. We suppose there exists $T=T(Q)$ with $0<T \leq+\infty$ such that (4.2) holds for $0<t<T$ while it fails for $t>T$.

Lemma 4.21. $(0<t<T)$ Let $D$ be a bounded domain in $\mathbb{C}$ and suppose $\Delta Q \geq 0$ in $D$. Then $\partial D \subset S_{t}^{*}$ implies $D \subset S_{t}^{*}$.

Proof. The assumption $\partial D \subset S_{t}^{*}$ means that $\widehat{Q}_{t}=Q$ on $\partial D$. We write $R_{0}=\widehat{Q}_{t}$, and let $R_{1}$ be the function which equals $Q$ in $D$ and equals $\widehat{Q}_{t}$ elsewhere. We observe that $R_{0} \leq R_{1} \leq Q$ on $\mathbb{C}$, while $R_{0}=R_{1}=Q$ on $\partial D$. Also, the function $R_{1}$ is subharmonic. Indeed, $\Delta R_{1}=\Delta Q \geq 0$ on $D$ (by assumption), and $\Delta R_{1}=\Delta R_{0} \geq 0$ on $\mathbb{C} \backslash \bar{D}$. It remains to observe that for $a \in \partial D$,

$$
R_{1}(a)=R_{0}(a) \leq \frac{1}{2 \pi} \int_{-\pi}^{\pi} R_{0}\left(a+\varepsilon \mathrm{e}^{\mathrm{i} \theta}\right) \mathrm{d} \theta \leq \frac{1}{2 \pi} \int_{-\pi}^{\pi} R_{1}\left(a+\varepsilon \mathrm{e}^{\mathrm{i} \theta}\right) \mathrm{d} \theta, \quad 0<\varepsilon<+\infty .
$$

We see that $R_{1}$ is subharmonic in $\mathbb{C}$, and the conclusion $R_{1}=R_{0}$ follows.

Corollary 4.22. If $Q$ is subharmonic in $\mathbb{C}$, then $\mathbb{C} \backslash S_{t}^{*}$ is connected.

A continuous function $h: \mathbb{C} \rightarrow \mathbb{R}$ is said to be nowhere harmonic if for every open set $D \subset \mathbb{C}$ the restriction $\left.h\right|_{D}$ fails to be harmonic.

Corollary 4.23. Suppose $Q$ is subharmonic in $\mathbb{C}$, and that $Q$ is nowhere harmonic. Then $S_{t_{0}}^{*} \subset S_{t}$ for all $t_{0}, t$ with $0<t_{0}<t<T$.

Proof. By Corollary 4.22, the set $\mathbb{C} \backslash S_{t_{0}}^{*}$ is connected, and so phull $\left(S_{t_{0}}^{*}\right)=S_{t_{0}}^{*}$. By Lemma4.19, then, we arrive at $\partial S_{t_{0}}^{*} \subset S_{t}$ for all $t$ with $t_{0}<t<T$. It remains to check that int $S_{t_{0}}^{*} \subset S_{t}$ for all $t$ with $t_{0}<t<T$. By Proposition 4.12, we just need to show that no point in int $S_{t_{0}}^{*}$ is $Q$-shallow with respect to $S_{t}^{*}$. This is guaranteed by the requirement that $Q$ be nowhere harmonic.

4.9. Convex potentials. We say that a convex function $q: \mathbb{C} \rightarrow \mathbb{R}$ is locally uniformly convex if

$$
|\xi|^{2} \Delta q(z)+\operatorname{Re}\left[\xi^{2} \partial^{2} q(z)\right] \geq \epsilon(z)|\xi|^{2}, \quad \xi \in \mathbb{C},
$$

for some continuous $\epsilon: \mathbb{C} \rightarrow] 0,+\infty\left[\right.$. For $C^{2}$-smooth $q$, this just says that the Hessian of $q$ is (strictly) positive definite everywhere.

In [18], Chapter V, coincidence sets for constrained obstacle problems are considered, and under suitable convexity assumptions, the coincidence set is simply connected with $C^{1, \alpha}$-smooth boundary (here, $0<\alpha<1$ ). The setting is the following. Suppose $\Omega$ is a strictly convex bounded $C^{\infty}$-smooth domain, and let $q: \bar{\Omega} \rightarrow \mathbb{R}$ be $C^{2}$-smooth and locally uniformly convex, with $q>0$ on $\partial \Omega$ and $\min _{\Omega} q<0$. Then, if we put $\varrho=0$ in the constrained obstacle problem (see Subsection 4.4), the coincidence set

$$
S_{\Omega, q}^{*}:=\left\{z \in \Omega: \operatorname{Obst}_{\Omega, 0}[q](z)=q(z)\right\}
$$


is non-empty, compact, simply connected, and equal to the closure of its interior. Moreover, if $q$ is $C^{2, \alpha}$-smooth for some $\alpha, 0<\alpha<1$, then the boundary $\partial S_{\Omega, q}^{*}$ is a $C^{1, \alpha^{\prime}}$-smooth Jordan curve, for some $\alpha^{\prime}, 0<\alpha^{\prime}<1$.

Applied to our setting (cf. Subsection 4.3), we get Theorem 4.24 below. Before we formulate the theorem, we note that if $Q: \mathbb{C} \backslash \mathbb{R}$ is convex, and (4.2) holds for some positive $t$, then $Q$ must grow faster (radially, the growth is at least linear), so that (4.2) holds for all positive reals $t$ (which makes $T=T(Q)=+\infty)$.

Theorem 4.24. $(0<t<T=+\infty)$ Suppose $Q: \mathbb{C} \rightarrow \mathbb{R}$ is $C^{2}$-smooth and locally uniformly convex with (4.2). Then the droplet $S_{t}$ is simply connected, and equal to the closure of its interior. Moreover, if $Q$ is $C^{2, \alpha}$-smooth for some $\alpha, 0<\alpha<1$, then $\partial S_{t}$ is a $C^{1, \alpha^{\prime}}$-smooth Jordan curve, for some $\alpha^{\prime}, 0<\alpha^{\prime}<1$.

Proof. We claim that for big enough $c$, the compact set

$$
\bar{\Omega}_{c}:=\left\{z \in \mathbb{C}: \widehat{Q}_{t}(z) \leq c\right\}
$$

is strictly convex with $C^{\infty}$-smooth boundary. In fact, we know from (4.4) and the fact that $\widehat{Q}_{t}$ has the form

$$
\widehat{Q}_{t}(z)=t \log |z|^{2}+h(z),
$$

where $h$ is real-valued, bounded, and harmonic in a neighborhood of infinity. As $c$ increases the sets $\bar{\Omega}_{c}$ cover bigger and bigger portions of the plane $\mathbb{C}$, and the boundary $\partial \bar{\Omega}_{c}$ is contained in a fixed neighborhood of infinity for big enough $c$. The equation defining the boundary is

$$
|z| \mathrm{e}^{h(z)}=\mathrm{e}^{c},
$$

and an argument using the harmonic conjugate of $h$ shows this equation may be written in the form

$$
\left|z+a_{0}+a_{1} z^{-1}+a_{2} z^{-2}+\ldots\right|=\mathrm{e}^{c},
$$

where the series converges for big $|z|$. In other words, using the inverse mapping, $\partial \bar{\Omega}_{c}$ is (for big c) the image of the circle $|z|=\mathrm{e}^{c}$ under a mapping

$$
z \mapsto z+b_{0}+b_{1} z^{-1}+b_{2} z^{-2}+\ldots,
$$

which also converges for big $|z|$. After rescaling by a factor of $\mathrm{e}^{-c}$, we are talking about the image of the unit circle $|z|=1$ under the mapping

$$
z \mapsto z+b_{0} \mathrm{e}^{-c}+b_{1} \mathrm{e}^{-2 c} z^{-1}+b_{2} \mathrm{e}^{-3 c} z^{-2}+\ldots,
$$

which for large values of $c$ constitutes a very slight perturbation of the circle $|z|=1$, and it is then easy to check that the domain inside the curve is strictly convex with $C^{\infty}$-smooth boundary. As a consequence, $\bar{\Omega}_{c}$ is strictly convex with $C^{\infty}$-smooth boundary for big $c$. To finish the proof, we observe that (cf. Lemma4.7)

$$
\operatorname{Obst}_{\Omega_{c}, 0}[q]+c=\operatorname{Obst}_{t}[Q] \text { on } \bar{\Omega}_{c},
$$

if $q=Q-c$. It is immediate that $S_{t}=S_{\Omega_{c}, q}$. The rest follows from Chapter $\mathrm{V}$ of [18].

\section{LOCAL DROPLETS}

5.1. Localization. We often localize the field $Q: \mathbb{C} \rightarrow \mathbb{R} \cup\{+\infty\}$ (which we assume to be lower semi-continuous) to a closed set $\Sigma \subset \mathbb{C}$ and write

$$
Q_{\Sigma}= \begin{cases}Q & \text { on } \Sigma \\ +\infty & \text { on } \mathbb{C} \backslash \Sigma .\end{cases}
$$

The function $Q_{\Sigma}$ is then also lower semi-continuous. We will assume that $Q_{\Sigma}$ meets the growth condition (4.2), (which is the $t$-scaled version of (2.6); in case $\Sigma$ is compact, this is automatically 
so irrespective of the behavior of $Q$ near infinity). To avoid triviality, we also need to require that $Q_{\Sigma}<+\infty$ on a set of positive area. We will refer to the closed set $\Sigma$ as a localization.

We will use the notation (which corresponds to the special parameter choice $t=1$ )

$$
\hat{\sigma}_{Q, \Sigma}=\hat{\sigma}[Q, \Sigma]:=\hat{\sigma}_{Q_{\Sigma}}, \quad S_{Q, \Sigma}=S[Q, \Sigma]:=\operatorname{supp} \hat{\sigma}[Q, \Sigma] ;
$$

this conforms with the convention to write $\hat{\sigma}_{Q}=\hat{\sigma}[Q]$ and $S_{Q}=S[Q]$. We will focus on the $t$-scaled variants $(0<t<+\infty)$

$$
\hat{\sigma}_{t}[Q, \Sigma]:=t \hat{\sigma}_{Q / t, \Sigma}, \quad S_{t}[Q, \Sigma]:=\operatorname{supp} \hat{\sigma}_{t}[Q, \Sigma]=\operatorname{supp} \hat{\sigma}[Q / t, \Sigma]=S[Q / t, \Sigma] .
$$

We shall also need the modified Robin constant

$$
\gamma_{t}^{*}(Q, \Sigma)=\gamma_{t}^{*}\left(Q_{\Sigma}\right)
$$

from Subsection 4.1

Lemma 5.1. $(0<t<+\infty)$ Suppose $\Sigma \subset \mathbb{C}$ is closed, and that $Q_{\Sigma}$ meets the growth condition (4.2), while $Q_{\Sigma}<+\infty$ holds on a set of positive area. Then $S_{t}[Q, \Sigma] \subset \Sigma$.

Proof. If the probability measure $\sigma_{0}:=\hat{\sigma}[Q / t, \Sigma]$ were to have support outside $\Sigma$, the corresponding energy

$$
I_{Q_{\Sigma} / t}\left[\sigma_{0}\right]=\int_{\mathbb{C}^{2}} \log \frac{1}{|\xi-\eta|^{2}} \mathrm{~d} \sigma_{0}(\xi) \mathrm{d} \sigma_{0}(\eta)+\frac{2}{t} \int_{\mathbb{C}} Q_{\Sigma} \mathrm{d} \sigma_{0}
$$

would necessarily equal $+\infty$, which does not agree with the energy minimizing property of the equilibrium measure.

We now compare two different localizations, one contained in the other.

Lemma 5.2. $(0<t<+\infty)$ Suppose $\Sigma_{1}, \Sigma_{2} \subset \mathbb{C}$ are closed with $\Sigma_{1} \subset \Sigma_{2}$, and that $Q_{\Sigma_{2}}$ meets the growth condition (4.2), while $Q_{\Sigma_{1}}<+\infty$ holds on a set of positive area. If $S_{t}\left[Q, \Sigma_{2}\right] \subset \Sigma_{1}$, then

$$
\hat{\sigma}_{t}\left[Q, \Sigma_{1}\right]=\hat{\sigma}_{t}\left[Q, \Sigma_{2}\right], \quad S_{t}\left[Q, \Sigma_{1}\right]=S_{t}\left[Q, \Sigma_{2}\right]
$$

Proof. For $j=1,2$, we write $\sigma_{j}=\hat{\sigma}\left[Q / t, \Sigma_{j}\right]$ and $Q_{j}=Q_{\Sigma_{j}} / t$. If $L_{Q}$ is as in (2.10), we then get that (since $\Sigma_{1} \subset \Sigma_{2}$ )

$$
L_{Q_{1}}(\xi, \eta)=L_{Q_{2}}(\xi, \eta), \quad(\xi, \eta) \in \Sigma_{1} \times \Sigma_{1}
$$

and so (since $\left.S_{t}\left[Q, \Sigma_{2}\right]=\operatorname{supp} \hat{\sigma}\left[Q / t, \Sigma_{2}\right]=\operatorname{supp} \sigma_{2} \subset \Sigma_{1}\right)$

$$
I_{Q_{1}}\left[\sigma_{2}\right]=\int_{\mathbb{C}^{2}} L_{Q_{1}}(\xi, \eta) \mathrm{d} \sigma_{2}(\xi) \mathrm{d} \sigma_{2}(\eta)=\int_{\mathbb{C}^{2}} L_{Q_{2}}(\xi, \eta) \mathrm{d} \sigma_{2}(\xi) \mathrm{d} \sigma_{2}(\eta)=I_{Q_{2}}\left[\sigma_{2}\right],
$$

where we have used the identity (2.11). As $Q_{1} \geq Q_{2}$, we have

$$
I_{Q_{1}}\left[\sigma_{1}\right]=\inf _{\sigma} I_{Q_{1}}[\sigma] \geq \inf _{\sigma} I_{Q_{2}}[\sigma]=I_{Q_{2}}\left[\sigma_{2}\right],
$$

where both infima run over $\sigma \in \operatorname{prob}_{c}(\mathbb{C})$. Combined with (5.1), this gives $I_{Q_{1}}\left[\sigma_{1}\right] \leq I_{Q_{1}}\left[\sigma_{1}\right]$, which is only possible if $\sigma_{1}=\sigma_{2}$, by Frostman's theorem (Theorem 2.4). Finally, if the measures coincide, their supports coincide as well.

The typical application of Lemma 5.1 will be when both $\Sigma_{1}$ and $\Sigma_{2}$ are compact. However, already the case when $\Sigma_{1}=S_{Q}$ and $\Sigma_{2}=\mathbb{C}$ is interesting.

Corollary 5.3. $(0<t<+\infty)$ If $Q$ meets the growth condition (4.2), then with $\Sigma:=S_{t}[Q]=S_{Q / t}$, we have

$$
\hat{\sigma}_{t}[Q]=\hat{\sigma}_{t}[Q, \Sigma], \quad S_{t}[Q, \Sigma]=S_{t}[Q]
$$


5.2. Local droplets and the obstacle problem. We let $\Sigma$ be a localization, and suppose that $Q<+\infty$ on a subset of $\Sigma$ with positive area. We require that $Q_{\Sigma}$ meets the growth condition (4.2) for a positive $t$, which is kept fixed for the moment (this requirement is void if $\Sigma \subset \mathbb{C}$ is compact). Then the Borel measure $\hat{\sigma}_{t}[Q, \Sigma]$ is a well-defined positive measure of total mass $t$, and its support $S_{t}[Q, \Sigma]$ is compact with $S \subset \Sigma$. The following lemma is immediate from Lemma 4.8 .

Lemma 5.4. Suppose $Q \in W^{2,1}(\operatorname{int} S)$ where $S=S_{t}[Q, \Sigma]$. Then $\hat{\sigma}_{t}[Q, \Sigma]$ is absolutely continuous in int $S$ and in fact

$$
\mathrm{d} \hat{\sigma}=\Delta Q \mathrm{~d} A \quad \text { on } \operatorname{int} S
$$

Proof. This follows from Lemma 4.8

We are led to the following three definitions.

Definition 5.5. Suppose $Q$ is in $W^{2,1}$ on a neighborhood of $S=S_{t}[Q, \Sigma]$. We say that $S$ is a local $(Q, t)$-droplet with localization $\Sigma$ if the following equality holds $\left(\hat{\sigma}=\hat{\sigma}_{t}[Q, \Sigma]\right)$ :

$$
\mathrm{d} \hat{\sigma}=1_{S} \Delta Q \mathrm{~d} A \text {. }
$$

Definition 5.6. A compact set $S \subset \mathbb{C}$ is a local $(Q, t)$-droplet if it is a local $(Q, t)$-droplet with respect to some localization $\Sigma$.

Definition 5.7. A compact set $S \subset \mathbb{C}$ is a global $(Q, t)$-droplet if it is a local $(Q, t)$-droplet with respect to the localization $\Sigma=\mathbb{C}$.

Remark 5.8. (a) There is at most one global $(Q, t)$-droplet $S$, as it is given by $S=S_{t}[Q]$. Theorem 4.10 guarantees that it exists under the growth requirement (4.2) and the additional regularity $Q \in W^{2, p}$ (this is true also if $Q$ is in $W^{2, p}$ only in a neighborhood of $S$ ). In contrast, there may exist several local $(Q, t)$-droplets.

(b) If the boundary $\partial S$ of the set $S=S[Q, \Sigma]$ has zero area, then $S$ is a local $(Q, t)$-droplet if and only if and only if $\hat{\sigma}=\hat{\sigma}_{t}[Q, \Sigma]$ is absolutely continuous.

(c) Two local $(Q, t)$-droplets $S_{1}, S_{2}$ cannot have the containment $S_{1} \subset S_{2}$ unless $S_{1}=S_{2}$.

(d) The point with the definition of local droplets is that we may focus on the support $S=\operatorname{supp} \hat{\sigma}$ rather than the (generally more complicated) equilibrium measure $\hat{\sigma}$ (with respect to the weight $\left.Q_{\Sigma}\right)$.

(e) In the above definition, it is possible to weaken the smoothness assumption on $Q$ to $W^{\Delta, 1}$ smoothness, which just asks that the function and its Laplacian are both locally integrable.

Proposition 5.9. If $S$ is a local $(Q, t)$-droplet with respect to some localization $\Sigma=\Sigma_{0}$, then it is a local $(Q, t)$-droplet with respect to the minimal localization $\Sigma=S$.

Proof. This follows from Lemma 5.2 with $\Sigma_{1}=S$ and $\Sigma_{2}=\Sigma_{0}$.

Remark 5.10. If $S=S_{t}[Q, \Sigma]$ is a local $(Q, t)$-droplet, then the associated measure $\hat{\sigma}=\hat{\sigma}_{t}[Q, \Sigma]$ is absolutely continuous. It is possible that the converse might be true (cf. Lemma 5.4 above). For the moment, we have a weaker statement. Suppose first that $Q$ is in $W^{2, p}$ in a neighborhood of $S$, for some $p, 1<p<+\infty$. The statement now runs as follows: if $\hat{\sigma}$ is absolutely continuous with density in $L^{p}$ for some $p, 1<p<+\infty$, then $S$ is a local $(Q, t)$-droplet. Indeed, from the properties of the 2D Hilbert transform, we get that the function

$$
\left.\widehat{\left(Q_{\Sigma}\right.}\right)_{t}(\xi)=\gamma_{t}^{*}(Q, \Sigma)-U^{\hat{\sigma}}(\xi),
$$

is in $W^{2, p}$ and from Proposition 4.5 we have that

$$
\widehat{\left(Q_{\Sigma}\right)_{t}}(\xi)=Q(\xi), \quad \xi \in S,
$$


so that by [18], p. 53, we get

$$
\Delta\left(\widehat{Q_{\Sigma}}\right)_{t}(\xi)=\Delta Q(\xi), \quad \xi \in S,
$$

as distributions, which leads to the desired result.

For a compact $S \subset \mathbb{C}$, we define the corresponding (weighted) logarithmic potential

$$
U^{\mathrm{Q}, S}(\xi)=\int_{S} \log \frac{1}{|\xi-\eta|^{2}} \Delta Q(\eta) \mathrm{d} A(\eta) .
$$

We have the following characterization of local $(Q, 1)$-droplets. We recall the notion of $Q$ shallow points from Subsection 4.6 .

Theorem 5.11. Suppose that $S \subset \Sigma \subset \mathbb{C}$, where $S$ is compact and $\Sigma$ closed, and that $Q$ is in $W^{2,1}$ in a neighborhood of $S$. Then $S$ is a local $(Q, t)$-droplet with localization $\Sigma$ if and only if:

(i) $\Delta Q \geq 0$ a.e. on $S$,

(ii) $S$ contains no $Q$-shallow points,

$$
\int_{S} \Delta Q \mathrm{~d} A=t
$$

(iv) $U^{S, Q}+Q=\gamma_{t}^{*}(Q, S)$ q.e. on $S$, for some real constant $\gamma_{t}^{*}(Q, S)$ (the modified Robin constant), and (v) $U^{S, Q}+Q \geq \gamma_{t}^{*}(Q, S)$ q.e. on $\Sigma$.

Proof. We first establish the necessity of conditions (i)-(iv). So, we suppose that $S$ is a local $(Q, t)$ droplet. As d $\hat{\sigma}=1_{S} \Delta Q \mathrm{~d} A$ is positive with mass $t$, and $S$ is its support set, conditions (i)-(iii) are necessary. The necessity of condition (iv) and (v) follows from Frostman's Theorem 2.6 (with $Q_{S} / t$ in place of $Q$, where $S$ is used as a localization).

We turn to the sufficiency of the conditions (i)-(v). We write $W:=c-U^{Q, S}$, where the constant $c=\gamma_{t}^{*}(Q, S)$ is as in (iv). By (iv), we then have $W=Q_{\Sigma}$ q.e. on $S$ while (v) gives $W \leq Q_{\Sigma}$ q.e. on C. By Lemma 4.1, we get that $W=\operatorname{Obst}_{t}\left[Q_{\Sigma}\right]$. Next, Proposition 4.2 and Corollary 4.4 show that

$$
\mathrm{d} \hat{\sigma}_{t}[Q, S]=\Delta W \mathrm{~d} A=-\Delta U^{Q, S} \mathrm{~d} A=1_{S} \Delta Q \mathrm{~d} A .
$$

So we have a local $(Q, t)$-droplet with localization $\Sigma$.

Remark 5.12. To characterize the local $(Q, t)$-droplets, we use the minimal localization $S$. We see that condition (v) becomes vacuous and may be removed.

Corollary 5.13. Suppose that $S \subset \Sigma \subset \mathbb{C}$, where $S$ is compact and $\Sigma$ closed, and that $Q$ is in $W^{2,1}$ in a neighborhood of $S$. Consider the function

$$
\widehat{Q}_{S}:=\gamma_{t}^{*}(Q, S)-U^{Q, S},
$$

where $\gamma_{t}^{*}(Q, S)$ is the constant in Theorem 5.11 Then $S$ is a local $(Q, t)$-droplet with localization $\Sigma$ if and only if:

(i) $\Delta Q \geq 0$ a.e. on $S$,

(ii) $S$ contains no $Q$-shallow points,

$$
\int_{S} \Delta Q \mathrm{~d} A=t
$$

(iv) $\widehat{Q}_{S}=Q$ q.e. on $S$, and

(v) $\widehat{Q}_{S} \leq Q$ q.e. on $\Sigma$.

Moreover, if (i)-(v) are assumed, then $\widehat{Q}_{S} \in \mathrm{Sub}_{t}(\mathbb{C})$ is harmonic on $\mathbb{C} \backslash S$, with asymptotics

$$
\widehat{Q}_{S}(z)=t \log |z|^{2}+\mathrm{O}(1) \quad \text { as }|z| \rightarrow+\infty \text {. }
$$

As a consequence, we have q.e.

$$
\widehat{Q}_{S}={\widehat{\left(Q_{\Sigma}\right.}}_{t}=\operatorname{Obst}_{t}\left[Q_{\Sigma}\right]
$$


Moreover, if, for some $p$ with $1<p<+\infty$, we have $Q \in W^{2, p}$ in a neighborhood of $S$, then $\widehat{Q}_{S} \in W^{2, p}$ as well.

Proof. It is clear from the properties of logarithmic potentials that $U^{Q, S}$ is subharmonic in $\mathbb{C}$ and harmonic in $\mathbb{C} \backslash S$, with the corresponding asymptotics at infinity as a consequence of condition (ii) of Theorem [5.11. Moreover, the properties of the 2D Hilbert transform show that if $Q \in W^{2, p}$ in a neighborhood of $S$, then $U^{Q, S} \in W^{2, p}$, for $1<p<+\infty$. These properties are then inherited by $\widehat{Q}_{S}$.

If there is some room to wiggle between the set $S_{t}[Q, \Sigma]$ and the localization $\Sigma$, then the set $S_{t}[Q, \Sigma]$ is automatically a local $(Q, t)$-droplet:

Theorem 5.14. Suppose $Q \in W^{2, p}$ for some $p, 1<p<+\infty$. If for a localization $\Sigma$ we have $S=S_{t}[Q, \Sigma] \subset$ int $\Sigma$, then $S$ is a local $(Q, t)$-droplet with localization $\Sigma$.

Proof. This is Theorem 4.10 for $Q_{\Sigma}$ in place of $Q$.

Remark 5.15.

The modified Robin constant $\gamma^{*}(Q, S)$ may be written out explicitly:

$$
\gamma_{t}^{*}(Q, S)=\frac{1}{t} \int_{S \times S} \log \frac{1}{|\xi-\eta|^{2}} \Delta Q(\xi) \Delta Q(\eta) \mathrm{d} A(\xi) \mathrm{d} A(\eta)+\int_{S} Q \Delta Q \mathrm{~d} A .
$$

5.3. Characterization of local droplets. We need the concept of local $Q$-droplets. We consider compact localizations $\Sigma$ only, which means that no requirement on $Q$ near infinity is needed, just that $Q: \mathbb{C} \rightarrow \mathbb{R} \cup\{+\infty\}$ is lower semi-continuous and has $Q<+\infty$ on a subset of $\Sigma$ with positive area. We recall the concept of a $(Q, t)$-droplet, which presupposed that $Q$ was $W^{2,1}$-smooth near S.

Definition 5.16. A compact set $S \subset \mathbb{C}$ is a (local) $Q$-droplet if it is a local $(Q, t)$-droplet for some $t$ with $0<t<+\infty$.

We see that Theorem 5.11 has the following consequence.

Corollary 5.17. Suppose that $S \subset \mathbb{C}$ is compact, and that $Q$ is in $W^{2,1}$ in a neighborhood of $S$. Then $S$ is a local Q-droplet if and only if:

(i) $\Delta Q \geq 0$ a.e. on $S$,

(ii) $S$ contains no $Q$-shallow points, and

(iii) $U^{S, Q}+Q$ is constant q.e. on $S$.

By Sobolev imbedding, we have $W^{2, p} \subset C^{1}$ for $2<p \leq+\infty$. The following characterization will prove useful later.

Proposition 5.18. $(0<t<+\infty)$ Suppose $S \subset \mathbb{C}$ is compact with $S=\operatorname{clos}$ int $S$, and that $Q \in W^{2, p}$ in a neighborhood of $S$, for some $p, 2<p<+\infty$. We then have:

(i) If $S$ is a local $Q-$ droplet, then $\bar{\partial}\left(U^{Q, S}+Q\right)=0$ on $S$.

(ii) If $S$ is connected and $\bar{\partial}\left(U^{Q, S}+Q\right)=0$ on $\partial S$, and if $S$ has no $Q$-shallow points, then $S$ is a local $Q$-droplet.

Proof. We first treat part (i). So, we assume that $S$ is a local $Q$-droplet. By Corollary $5.17 U^{Q, S}+Q$ is constant q.e. on $S$. As both $Q$ and $U^{Q, S}$ are in $W^{2, p}$ in a neighborhood of $S$, we conclude from [18], p. 53, that $\bar{\partial}\left(U^{Q, S}+Q\right)=0$ a.e. on $S$. By Sobolev imbedding, $\bar{\partial}\left(U^{Q, S}+Q\right)$ is continuous in a neighborhood of $S$, and so $\bar{\partial}\left(U^{Q, S}+Q\right)=0$ on int $S$ and a fortiori (by the topological assumption) on $S$.

We turn to part (ii). Consider the function $F:=\bar{\partial}\left(U^{Q, S}+Q\right)$, which is in $W^{1, p}$ in a neighborhood of $S$, and therefore continuous. We have

$$
\partial F=\Delta\left(U^{Q, S}+Q\right)=-1_{S} \Delta Q+\Delta Q=0 \text { a.e. on } S .
$$


Hence $F$ is conjugate holomorphic in the interior of $S$ and since $F=0$ on the boundary, we have $F \equiv 0$ on $S$. If $S$ is connected, then this implies that $U_{S}+Q$ is constant on $S$, so by Corollary 5.17, $S$ is a local $Q$-droplet.

\section{Chains of LOCAL DRoplets}

6.1. A partial ordering of local droplets. We recall that $S$ is a local $Q$-droplet if it is a local $(Q, t)$-droplet for some $t$ with $0<t<+\infty$. For the concept to make sense, we need to ask that $Q: \mathbb{C} \rightarrow \mathbb{R} \cup\{+\infty\}$ is lower semi-continuous and $W^{2,1}$-smooth near $S$. Given a local $Q$-droplet $S$, the corresponding value of (the evolution parameter) $t$ is easily calculated:

$$
t=t(Q, S):=\int_{S} \Delta Q \mathrm{~d} A .
$$

We note that by Corollary 5.17, $\Delta Q \geq 0$ on $S$. To simplify the presentation, we shall assume that $Q$ is $W^{2, p}$-smooth in $\mathbb{C}$ for some $p, 1<p<+\infty$.

Lemma 6.1. Let $S_{2}$ be a local $Q$-droplet, with $t_{2}=t\left(Q, S_{2}\right)$. If $t_{1}$ has $0<t_{1}<t_{2}$ we put $S_{1}:=S_{t_{1}}\left[Q, S_{2}\right]$. Then $S_{1}$ is a local $Q$-droplet, with $t_{1}=t\left(Q, S_{1}\right)$.

Proof. We should study the measure $\sigma_{1}:=\sigma_{t_{1}}\left[Q, S_{2}\right]$, which by Proposition 4.2 and Corollary 4.4 is obtained from $\mathrm{Obst}_{t_{1}}\left[Q_{S_{2}}\right]$ by applying the Laplacian. From $t_{1}<t_{2}$ and the definition of the obstacle problem, we see that

$$
\operatorname{Obst}_{t_{1}}\left[Q_{S_{2}}\right] \leq \operatorname{Obst}_{t_{2}}\left[Q_{S_{2}}\right]=\widehat{Q}_{S_{2}}
$$

where we use Corollary 5.13 to get the rightmost identity. A moments reflection, using that $\widehat{Q}_{S_{2}} \leq Q$, reveals that in fact

$$
\operatorname{Obst}_{t_{1}}\left[Q_{S_{2}}\right]=\operatorname{Obst}_{t_{2}}\left[\widehat{Q}_{S_{2}}\right] \text {. }
$$

Since $\Delta Q$ is in $L^{p}$ locally, $\widehat{Q}_{S_{2}}$ is $W^{2, p}$-smooth, and by Theorem 4.10 with $\widehat{Q}_{S_{2}}$ in place of $Q$, we get that $S_{1}$ is a local $Q$-droplet.

Lemma 6.1 allows us to introduce a partial ordering in the set of all local $Q$-droplets.

Definition 6.2. Let $S_{1}, S_{2}$ be two local $Q$-droplets, and write $t_{j}=t\left(Q, S_{j}\right), j=1,2$. We write $S_{1}<S_{2}$ if $S_{1} \subset S_{2}$ and $S_{1}=S_{t_{1}}\left[Q, S_{2}\right]$.

Remark 6.3. (a) In other words, $S_{1}<S_{2}$ if $S_{1}, S_{2}$ are local $Q$-droplets and $S_{1}$ is a local $\left(Q, t_{1}\right)$-droplet with localization $S_{2}$, where $t_{1}=t\left(Q, S_{1}\right)$.

(b) It follows from the definition that if $S_{1}, S_{2}$ are $Q$-droplets with $S_{1}<S_{2}$, then $t\left(Q, S_{1}\right) \leq t\left(Q, S_{2}\right)$.

(c) If $S_{1} \prec S_{2}$ and $S_{2}<S_{1}$ for two local $Q$-droplets, then $S_{1} \subset S_{2}$ and $S_{2} \subset S_{2}$, and so $S_{1}=S_{2}$.

Proposition 6.4. Let $S_{1}, S_{2}$ be two local $Q$-droplets with $S_{1} \subset S_{2}$. Then $S_{1} \prec S_{2}$ if and only if $\widehat{Q}_{S_{1}} \leq Q$ holds on $S_{2}$.

Proof. This follows from Corollary 5.13. After all, for local $Q$-droplets we do not need to check conditions (i)-(iv); only (v) remains. Moreover, by continuity and the fact that $Q$-droplets lack $Q$-shallow points, the q.e. statements hold everywhere.

Lemma 6.5. For a local $Q$-droplet $S$, we have $S<S$.

Proof. This follows from the definition of the " $<$ " relation together with Proposition 5.9

There is one more property we need to check to show that " $<$ " defines a partial ordering.

Lemma 6.6. If $S_{1}, S_{2}, S_{3}$ are three local Q-droplets with $S_{1}<S_{2}$ and $S_{2}<S_{3}$, then $S_{1}<S_{3}$. 
Proof. If we use that $S_{2}<S_{3}$, we see from Lemma 6.1 that $S_{t_{1}}\left[Q, S_{3}\right]$ is a local $Q$-droplet with

$$
S_{t_{1}}\left(Q, S_{3}\right) \subset S_{2}=S_{t_{2}}\left(Q, S_{3}\right) .
$$

Using that $S_{1}<S_{2}$, we appeal to Lemma 5.2 , and get

$$
S_{t_{1}}\left[Q, S_{3}\right]=S_{t_{1}}\left[Q, S_{2}\right]=S_{1}
$$

so that $S_{1} \prec S_{3}$, as claimed.

Remark 6.7. $S_{1} \subset S_{2}$ does not imply $S_{1} \prec S_{2}$. For example, suppose $Q$ has two global minima at the points 0 and 2, and suppose the minima are non-degenerate. Consider

$$
S_{1}:=S_{t_{1}}\left(Q, \Sigma_{1}\right), \quad S_{2}:=S_{t_{2}}\left(Q, \Sigma_{2}\right), \quad \text { where } \quad 0<t_{1} \ll t_{2} \ll 1,
$$

with $\Sigma_{1}=\overline{\mathrm{D}}(0,1)$ and $\Sigma_{2}=\overline{\mathrm{D}}(0,3)$. Then $S_{1} \subset S_{2}$ but $S_{1} \nprec S_{2}$. This is easy to see using the characterization of Proposition 6.4.

6.2. A comparison principle. We keep the setting of the previous subsection. We recall the definition of the polynomially convex hull phull $(E)$ of a compact set $E$ from Subsection 4.7 The set phull $(E) \backslash E$ is the union of all the bounded components of $\mathbb{C} \backslash E$.

Proposition 6.8. Suppose $S_{1}, S_{2}$ are two local Q-droplets with $S_{1} \subset S_{2}$. We then have $\widehat{Q}_{S_{2}} \leq \widehat{Q}_{S_{1}}$ on phull $\left(S_{1}\right)$, with equality on $S_{1}$. Moreover, if for some $z_{0} \in \operatorname{int}\left[\right.$ phull $\left.\left(S_{1}\right)\right]$ we have $\widehat{Q}_{S_{2}}\left(z_{0}\right)=\widehat{Q}_{S_{1}}\left(z_{0}\right)$, then $\widehat{Q}_{S_{2}}=\widehat{Q}_{S_{1}}$ holds on the component of $\operatorname{int}\left[\right.$ phull $\left.\left(S_{1}\right)\right]$ that contains $z_{0}$.

Proof. The difference $\widehat{Q}_{S_{2}}-\widehat{Q}_{S_{1}}$ is in $W^{2, p}$ and therefore continuous, and it is subharmonic, as

$$
\Delta\left[\widehat{Q}_{S_{2}}-\widehat{Q}_{S_{1}}\right]=1_{S_{2} \backslash S_{1}} \Delta Q \geq 0 \text { a.e. on } \mathbb{C} \text {. }
$$

Moreover, by Corollary 5.13, $\widehat{Q}_{S_{2}}=Q$ on $S_{2}$ and $\widehat{Q}_{S_{1}}=Q$ on $S_{1}$, and so $\widehat{Q}_{S_{2}}-\widehat{Q}_{S_{1}}=0$ on $S_{1}$ as $S_{1} \subset S_{2}$. The inequality $\widehat{Q}_{S_{2}}-\widehat{Q}_{S_{1}} \leq 0$ now follows from the maximum principle. The last assertion follows from the strong maximum principle.

We see that a local $Q$-droplet $S_{2}$ with $S_{1}<S_{2}$ does not grow in the direction of the interior holes of $S_{1}$ :

Corollary 6.9. Suppose $S_{1}, S_{2}$ are two local $Q$-droplets with $S_{1} \prec S_{2}$. We then have $\left[S_{2} \backslash S_{1}\right] \cap$ phull $\left(S_{1}\right)=$ $\emptyset$ and $\widehat{Q}_{S_{2}}=\widehat{Q}_{S_{1}}$ on $\operatorname{phull}\left(S_{1}\right)$.

Proof. If $S_{1}<S_{2}$ we have $\widehat{Q}_{S_{1}} \leq Q$ on $S_{2}$ (cf. Proposition 6.4), and we also have $\widehat{Q}_{S_{2}}=Q$ on $S_{2}$ (cf. Corollary 5.13). In view of Proposition 6.8, it follows that if $z_{0} \in S_{2} \cap$ phull( $\left.S_{1}\right)$, then $\widehat{Q}_{S_{2}}\left(z_{0}\right)=\widehat{Q}_{S_{1}}\left(z_{0}\right)$. So, if $z_{0} \in S_{2} \cap \operatorname{int}\left[\right.$ phull( $\left.\left(S_{1}\right)\right]$, another application of Proposition 6.8 shows that $\widehat{Q}_{S_{2}}=\widehat{Q}_{S_{1}}$ holds on the component of int $\left[\right.$ phull( $\left.\left(S_{1}\right)\right]$ which contains $z_{0}$. Taking the Laplacian, we find that $1_{S_{1}} \Delta Q=1_{S_{2}} \Delta Q$ a.e. on the component $\operatorname{Comp}\left(z_{0}\right)$ of int [phull( $\left.\left(S_{1}\right)\right]$ which contains $z_{0}$, which leads to

$$
S_{1} \cap \operatorname{Comp}\left(z_{0}\right)=S_{2} \cap \operatorname{Comp}\left(z_{0}\right) .
$$

Since $z_{0} \in S_{2}$ we also must have $z_{0} \in S_{1}$. We conclude that $\left[S_{2} \backslash S_{1}\right] \cap \operatorname{int}\left[\right.$ phull $\left.\left(S_{1}\right)\right]=\emptyset$, and $a$ fortiori $\left[S_{2} \backslash S_{1}\right] \cap$ phull $\left(S_{1}\right)=\emptyset$. But then $\widehat{Q}_{S_{2}}-\widehat{Q}_{S_{1}}$ is harmonic in int [phull( $\left.\left(S_{1}\right)\right]$ and vanishes on $\partial\left[\right.$ phull $\left.\left(S_{1}\right)\right] \subset \partial S_{1} \subset S_{1}$, and the conclusion $\widehat{Q}_{S_{2}}-\widehat{Q}_{S_{1}}=0$ on phull $\left(S_{1}\right)$ is immediate. 
6.3. Domination chains of local droplets. We are interested in chains of local $Q$-droplets.

Definition 6.10. A domination chain of local $Q$-droplets is a (continuously indexed) family of $Q$-droplets $\left\{S_{t}\right\}_{t}$, where the index $t$ ranges over a nonempty interval $I \subset \mathbb{R}_{+}$, with left endpoint 0 , such that $t=t\left(Q, S_{t}\right)$ and

$$
t_{1} \leq t_{2} \Longleftrightarrow S_{t_{1}}<S_{t_{2}} \text {. }
$$

The domination chain is terminating if the interval $I$ is given by $0<t \leq t_{*}$, for some $T_{*}$ with $0<t_{*}<+\infty$, and non-terminating if it is given by $0<t<t_{*}$ for some $t_{*}$ with $0<t_{*} \leq+\infty$. In case the domination chain is terminating, we say that it terminates at $S_{t_{*}}$.

Lemma 6.11. Given a local $Q$-droplet $S_{*}$, there is exactly one domination chain of local $Q$-droplets that terminates at $S_{*}$.

Proof. By Lemma 6.1, $S_{t}:=S_{t}\left[Q, S_{*}\right]$ for $0<t \leq t_{*}:=t\left(Q, S_{*}\right)$ defines a continuously indexed collection of local $Q$-droplets, and by Lemma 5.2 it is a (terminating) domination chain. Finally, if $S_{\sharp}$ is a local $Q$-droplet with $S_{\sharp}<S_{*}$, then by definition, it is of the form $S_{\sharp}=S_{t_{\sharp}}\left[Q, S_{*}\right]$ with $t_{\sharp}:=t\left(Q, S_{\sharp}\right) \leq t_{*}$, so the domination chain is unique.

6.4. Maximal domination chains of local $Q$-droplets. We keep the setting of the previous subsection. We shall need the concept of a maximal domination chain of local $Q$-droplets.

Definition 6.12. A domination chain of $Q$-droplets is maximal if it is contained in no larger domination chain of local $Q$-droplets.

Maximal domination chains of $Q$-droplets can be either terminating or non-terminating. If the chain is indexed by the unbounded interval $I=\mathbb{R}_{+}$then it is automatically non-terminating. If the chain is indexed by a bounded interval, then it can be non-terminating only if the droplets develop "arms" or "islands" that tend to infinity:

Theorem 6.13. Let $\left\{S_{t}\right\}_{t \in I}$ be a maximal non-terminating domination chain of local $Q$-droplets. Then the union

$$
S_{\cup}:=\bigcup_{t \in I} S_{t}
$$

is an unbounded subset of $\mathbb{C}$.

Proof. We suppose $S_{\cup}$ is bounded, and form $S_{*}=\operatorname{clos} S_{\cup}$, which is then compact. We are to show that the non-terminating domination chain $\left\{S_{t}\right\}_{t \in I}$ cannot be maximal. The interval $I$ is given by $0<t<t_{*}$ for some $t_{*}$ with $0<t_{*}<+\infty$. For $t \in I$, we let $\sigma_{t}$ be the positive measure $\mathrm{d} \sigma_{t}=1_{S_{t}} \Delta Q \mathrm{~d} A$, which has total mass $\left\|\sigma_{t}\right\|=t$. Let $\sigma_{*}$ be given by $\mathrm{d} \sigma_{*}=1_{S_{\cup}} \Delta Q \mathrm{~d} A$, which has total mass $\left\|\sigma_{*}\right\|=t_{*}$. Then $\sigma_{t} \rightarrow \sigma_{*}$ in norm as $t \rightarrow t_{*}$, and in fact the corresponding densities converge in $L^{p}$ :

$$
1_{S_{t}} \Delta Q \mathrm{~d} A \rightarrow 1_{S_{\cup}} \Delta Q \mathrm{~d} A \quad \text { in } L^{p}(\mathbb{C}) \text { as } t \rightarrow t_{*} .
$$

By the well-known properties of the 2D Hilbert transform, we find that the associated potentials converge in $W^{2, p}: U^{Q, S_{t}} \rightarrow U^{Q, S_{\cup}}$ as $t \rightarrow t_{*}$. Also, we easily check that if the constants $\gamma^{*}\left(Q, S_{t}\right)$ and $\gamma^{*}\left(Q, S_{\cup}\right)$ are as in (5.3), we have $\gamma^{*}\left(Q, S_{t}\right) \rightarrow \gamma^{*}\left(Q, S_{\cup}\right)$ as $t \rightarrow t_{*}$. As a consequence,

$$
\widehat{Q}_{S_{t}}=\gamma_{t}^{*}\left(Q, S_{t}\right)-U^{Q, S_{t}} \rightarrow \widehat{Q}_{S_{\cup}}=\gamma_{t_{*}}^{*}\left(Q, S_{t}\right)-U^{Q, S_{\cup}} \quad \text { in } W^{2, p} \quad \text { as } t \rightarrow t_{*} .
$$

By Sobolev imbedding the convergence is locally uniform. Since $\widehat{Q}_{S_{t}}=Q$ on $S_{t}$ we get that $\widehat{Q}_{S_{\cup}}=Q$ on $S_{\cup}$. By continuity, then, we find that $\widehat{Q}_{S_{\cup}}=Q$ on $S_{*}=\operatorname{clos} S_{\cup}$. Next, by [18], p. 53, we see that $\Delta \widehat{Q}_{S_{\cup}}=\Delta Q$ a.e. on $S_{*}$, that is, $1_{S_{U}} \Delta Q=\Delta Q$ a.e. on $S_{*}$. Expressed differently, we have $1_{S_{\cup}} \Delta Q=1_{S_{*}} \Delta Q$ as elements of $L^{p}(\mathbb{C})$. In particular, $\Delta Q \geq 0$ holds a.e. on $S_{*}$. By construction, $S_{*}$ has no $Q$-shallow points, a property this set inherits from the individual droplets $S_{t}, t \in I$. In view of Corollary5.17, $S_{*}$ is a local $Q$-droplet. It remains to show that we may add $S_{*}$ as a terminal local $Q$-droplet for the domination chain, thereby defeating the maximality of the non-terminating 
domination chain. To this end, it suffices to obtain that $S_{t}<S_{*}$ for $t \in I$. We pick a $t^{\prime}$ with $t<t^{\prime}<t_{*}$, and use $S_{t}<S_{t^{\prime}}$ to deduce that $\widehat{Q}_{S_{t}} \leq Q$ on $S_{t^{\prime}}$ (Proposition 6.4). By letting $t^{\prime} \rightarrow t_{*}$, we get that $\widehat{Q}_{S_{t}} \leq Q$ on $S_{\cup}$, and by continuity that $\widehat{Q}_{S_{t}} \leq Q$ on $S_{*}$. By Proposition 6.4 this means that $S_{t}<S_{*}$. The proof is finished.

The following definition is useful.

Definition 6.14. A local $Q$-droplet $S$ is maximal if for any other local $Q$-droplet $S^{\prime}$ the relation $S<S^{\prime}$ implies that $S=S^{\prime}$.

Corollary 6.15. A maximal domination chain $\left\{S_{t}\right\}_{t \in I}$ of local $Q$-droplets either terminates at a maximal local $Q$-droplet, or is non-terminating, in which case the set $S_{\cup}$ of Theorem 6.13 is unbounded.

6.5. Richardson's formula. We keep the setting of the previous two subsections. We would like to understand the flow evolution $t \mapsto S_{t}$ of domination chains (or containment chains, see the next subsection) of local $Q$-droplets. A natural way to do this is to analyze the effect of the flow when we use harmonic functions as test function (i.e., we calculate "harmonic moments").

Proposition 6.16. Suppose $S, S^{\prime}$ are two local $Q$-droplets with $S \subset S^{\prime}$. Then for all $h \in W^{2,1}(\mathbb{C})$ (local Sobolev class) that are harmonic in $\mathbb{C} \backslash S$ and bounded near infinity, we have (with $t=t(Q, S)$ and $\left.t^{\prime}=t\left(Q, S^{\prime}\right)\right)$

$$
\int_{S^{\prime} \backslash S} h \Delta Q \mathrm{~d} A=\left(t^{\prime}-t\right) h(\infty)
$$

Proof. The formula holds for constant $h$, by the choice of $t, t^{\prime}$. So, by subtracting a constant, we may take $h(\infty)=0$. We have

$$
\int_{S} h \Delta Q \mathrm{~d} A=\int_{\mathbb{C}} h 1_{S} \Delta Q \mathrm{~d} A=\int_{\mathbb{C}} h \Delta \widehat{Q}_{S} \mathrm{~d} A=\int_{\mathbb{C}} \widehat{Q}_{S} \Delta h \mathrm{~d} A,
$$

and the analogous identity holds for $S^{\prime}$ as well. By forming the difference between (6.1) for $S$ and $S^{\prime}$ we see that

$$
\int_{S^{\prime} \backslash S} h \Delta Q \mathrm{~d} A=\int_{\mathbb{C}}\left[\widehat{Q}_{S^{\prime}}-\widehat{Q}_{S}\right] \Delta h \mathrm{~d} A=0,
$$

because $\Delta h=0$ on $\mathbb{C} \backslash S$ while $\widehat{Q}_{S^{\prime}}-\widehat{Q}_{S}=Q-Q=0$ on $S$ (see, e.g., Corollary 5.13). To finish the proof, we just need to justify (6.1). By Green's formula, we have

$$
\int_{\mathbb{D}(0, R)}\left[h \Delta \widehat{Q}_{S}-\widehat{Q}_{s} \Delta h\right] \mathrm{d} A=2 \int_{\mathbb{T}(0, R)}\left[h \partial_{n} \widehat{Q}_{S}-\widehat{Q}_{S} \partial_{h} h\right] \mathrm{d} s,
$$

where $\mathrm{d} s$ is normalized arc length (i.e., arc length divided by $2 \pi$ ) and $\partial_{n}$ is the exterior normal derivative. Next, we observe that as $|z| \rightarrow+\infty$, we have the asymptotics

$$
h=\mathrm{O}\left(|z|^{-1}\right), \quad|\nabla h|=\mathrm{O}\left(|z|^{-2}\right), \quad \widehat{Q}_{S}=\mathrm{O}(\log |z|), \quad\left|\nabla \widehat{Q}_{S}\right|=\mathrm{O}\left(|z|^{-1}\right),
$$

because both $h$ and $\widehat{Q}_{S}$ are harmonic in $\mathbb{C} \backslash S$ with given asymptotical behavior. By letting $R \rightarrow+\infty$ in (6.2), we obtain (6.1). The proof is complete.

Corollary 6.17. Suppose $S, S^{\prime}$ are two local $Q$-droplets with $S \subset S^{\prime}$. Also suppose that the interior int $S$ has finitely many components. Then for all $h$ continuous and bounded in $\mathbb{C} \backslash$ int $S$, which are harmonic in $\mathbb{C} \backslash$ clos int $S$, we have (with $t=t(Q, S)$ and $t^{\prime}=t\left(Q, S^{\prime}\right)$ )

$$
\int_{S^{\prime} \backslash S} h \Delta Q \mathrm{~d} A=\left(t^{\prime}-t\right) h(\infty) \text {. }
$$

Proof. By Mergelyan-type approximation we can find a sequence of bounded $C^{\infty}$-smooth functions $h_{n}$ that are are harmonic in $\mathbb{C} \backslash \operatorname{clos}$ int $S$, such that $h_{n} \rightarrow h$ uniformly on $\mathbb{C} \backslash \operatorname{int} S$ as $n \rightarrow+\infty$. The assertion now follows from Proposition 6.16. 
6.6. Differential form of Richardson's formula. We keep the setting of the previous subsections, and introduce the concept of a containment chain.

Definition 6.18. A containment chain of local $Q$-droplets is a (continuously indexed) family of $Q$-droplets $\left\{S_{t}\right\}_{t}$, where the index $t$ ranges over a nonempty interval $I \subset \mathbb{R}_{+}$, with left endpoint 0 , such that $t=t\left(Q, S_{t}\right)$ and

$$
t_{1} \leq t_{2} \Longleftrightarrow S_{t_{1}} \subset S_{t_{2}} \text {. }
$$

Let $\left\{S_{t}\right\}_{t \in I}$ be a containment chain of local $Q$-droplets, and let $t_{*}$ denote the right endpoint of $I$. Let $I^{-}$be the interval obtained from $I$ by removal of $t_{*}$ (if $t_{*} \notin I$, we put $I^{-}:=I$ ).

Lemma 6.19. The map $t \mapsto S_{t}, t \in I$, is continuous in the Hausdorff metric except for a countable subset of the interval $I$.

Proof. For $t_{0} \in I^{-}$, we form the compact sets

$$
S_{t_{0}}^{-}=\operatorname{clos} \bigcup_{t: t<t_{0}} S_{t}, \quad S_{t_{0}}^{+}=\bigcap_{t: t>t_{0}} S_{t} .
$$

Then $S_{t}^{-} \subset S_{t} \subset S_{t}^{+}$holds for each for $t \in I^{-}$. Note that $S_{t_{0}}^{-}$is well-defined also when $t_{0}=t_{*}$. For $t \in I^{-}$, we put

$$
\delta^{-}(t):=\max _{z \in S_{t}} \operatorname{dist}_{\mathbb{C}}\left(z, S_{t}^{-}\right), \quad \delta^{+}(t):=\max _{z \in S_{t}^{+}} \operatorname{dist}_{\mathbb{C}}\left(z, S_{t}\right) .
$$

Next, for positive $\epsilon$, we consider the sets

$$
D_{\epsilon}^{-}:=\left\{t \in I^{-}: \delta^{-}(t)>\epsilon\right\}, \quad D_{\epsilon}^{+}:=\left\{t \in I^{-}: \delta^{+}(t)>\epsilon\right\} .
$$

We argue that for each positive $\epsilon$, the sets $D_{\epsilon}^{-}, D_{\epsilon}^{+}$are countable and that the only possible accumulation point is $t_{*}$ (and if $t_{*}$ is an accumulation point, then the set $S_{t_{*}}^{-}$must be unbounded). Indeed, if, for some $t^{\prime}$ with $0<t^{\prime}<t_{*}$, the set $\left.\left.D_{\epsilon}^{-} \cap\right] 0, t^{\prime}\right]$ has $N=N\left(t^{\prime}, \epsilon\right)$ elements, then the local $Q$-droplet $S_{t^{\prime}}$ contains $N$ points which are $\epsilon$-separated (the distance between any two different points is at least $\epsilon$ ). We get an effective bound on $N$ in terms of the diameter of the compact set $S_{t^{\prime}}$. The analogous argument applies to $D_{\epsilon}^{+}$in place of $D_{\epsilon}^{-}$.

We let $\omega_{\infty}^{(t)}$ denote harmonic measure for the open set $\mathbb{C} \backslash S_{t}$ with respect to the point at infinity. This is a probability measure whose support is contained in $\partial$ phull $\left(S_{t}\right) \subset \partial S_{t}$ (the effect on a test function is that we get the value at infinity of the harmonic extension).

Proposition 6.20. Suppose the map $t \mapsto S_{t}, t \in I$, is right continuous at $t_{0} \in I^{-}$. Suppose moreover that $\operatorname{int} S_{t_{0}}$ has finitely many components and that $S_{t_{0}}=\operatorname{clos} \operatorname{int} S_{t_{0}}$. Then for all $g \in C(\mathbb{C})$ we have

$$
\lim _{t \rightarrow t_{0}^{+}} \frac{1}{t-t_{0}} \int_{S_{t} \backslash S_{t_{0}}} g \Delta Q \mathrm{~d} A=\int_{\partial S_{t_{0}}} g d \omega_{\infty}^{\left(t_{0}\right)},
$$

that is, we have the weak-star convergence of measures

$$
\lim _{t \rightarrow t_{0}^{+}} \frac{1}{t-t_{0}} 1_{S_{t} \backslash S_{t_{0}}} \Delta Q \mathrm{~d} A=\mathrm{d} \omega_{\infty}^{\left(t_{0}\right)} .
$$

Proof. Let $h$ denote the function which coincides with $g$ on $S_{t_{0}}$ and extends harmonically (and boundedly) to $\mathbb{C} \backslash S_{t}$, so that in particular

$$
h(\infty)=\int_{\partial S_{t_{0}}} g d \omega_{\infty}^{\left(t_{0}\right)} .
$$

Then $h$ is continuous and bounded in $\mathbb{C}$ (see, e.g. [10] for a discussion of the Dirichlet problem). Since $S_{t_{0}}=$ clos int $S_{t_{0}}$, Corollary 6.17 applied to to $h$ gives

$$
\frac{1}{t-t_{0}} \int_{S_{t} \backslash S_{t_{0}}} g \Delta Q \mathrm{~d} A=h(\infty)+\frac{1}{t-t_{0}} \int_{S_{t} \backslash S_{t_{0}}}(g-h) \Delta Q \mathrm{~d} A .
$$


It remains to show that the last term on the right hand side tends to zero as $t \rightarrow t_{0}$. This follows from the fact that $h(z)-g(z) \rightarrow 0$ as $z \rightarrow \partial S_{t_{0}}$ and that $S_{t} \searrow S_{t_{0}}$ by the right continuity assumption.

Remark 6.21. Proposition 6.20 states that (under regularity assumptions) the infinitesimal growth of the local $Q$-droplets is in the exterior direction only. If the containment chain of local $Q$-droplets were to grow in the direction of the internal holes, the containment chain could not possibly be a domination chain (cf. Corollary 6.9).

6.7. Richardson's inequality. We now show that under modest regularity conditions, containment chains of local $Q$-droplets are in fact domination chains.

Theorem 6.22. Suppose $S, S^{\prime}$ are two local $Q$-droplets, with $S \subset S^{\prime}$. Then the following are equivalent: (i) $S<S^{\prime}$.

(ii) For all real-valued functions $h \in W^{2,1}(\mathbb{C})$ (local Sobolev class) that are subharmonic in $\mathbb{C} \backslash S$, harmonic in $\mathbb{C} \backslash S^{\prime}$, and bounded near infinity, we have (with $t=t(Q, S)$ and $t^{\prime}=t\left(Q, S^{\prime}\right)$ )

$$
\left(t^{\prime}-t\right) h(\infty) \leq \int_{S^{\prime} \backslash S} h \Delta Q \mathrm{~d} A .
$$

Proof. We first show that (i) $\Longrightarrow$ (ii). We note that the inequality is an equality when $h$ is constant (see, e.g., Proposition 6.16). This allows us to restrict our attention to $h$ with $h(\infty)=0$. As in the proof of Richardson's formula (Proposition 6.16), we find that

$$
\int_{S^{\prime} \backslash S} h \Delta Q \mathrm{~d} A=\int_{\mathbb{C}}\left[\widehat{Q}_{S^{\prime}}-\widehat{Q}_{S}\right] \Delta h \mathrm{~d} A=\int_{S^{\prime} \backslash S}\left[\widehat{Q}_{S^{\prime}}-\widehat{Q}_{S}\right] \Delta h \mathrm{~d} A \geq 0,
$$

since $\widehat{Q}_{S} \leq Q=\widehat{Q}_{S^{\prime}}$, by Proposition 6.4 and Corollary 5.13

We turn to the implication (ii) $\Longrightarrow$ (i). We take $h(\infty)=0$, and get (as above) from (ii) that

$$
0 \leq \int_{S^{\prime} \backslash S} h \Delta Q \mathrm{~d} A=\int_{S^{\prime} \backslash S}\left[\widehat{Q}_{S^{\prime}}-\widehat{Q}_{S}\right] \Delta h \mathrm{~d} A .
$$

The question now is what kind of functions $\Delta h$ are possible here. We have automatically $\Delta h \in$ $L^{1}\left(S^{\prime}\right)$ while $\Delta h \geq 0$ a.e. on $S^{\prime} \backslash S$. We also need to impose that

$$
\int_{S^{\prime}} \Delta h \mathrm{~d} A=0,
$$

as a consequence of the behavior of $h$ near infinity. In fact, any real-valued function $g \in L^{q}\left(S^{\prime}\right)$ for some $q$ with $1<q<+\infty$ with $g \geq 0$ a.e. on $S^{\prime} \backslash S$ and

$$
\int_{S^{\prime}} g \mathrm{~d} A=0
$$

is of the form $g=\Delta h$ for an $h$ as in (ii) with $h(\infty)=0$. It follows from (6.3) that

$$
0 \leq \int_{S^{\prime} \backslash S}\left[\widehat{Q}_{S^{\prime}}-\widehat{Q}_{S}\right] g \mathrm{~d} A \text {. }
$$

As it is easy to fulfill (6.4) by placing an $L^{q}$-integrable negative mass on $S$ to compensate for the positive mass on $S^{\prime} \backslash S$, on $S^{\prime} \backslash S$ the function $g$ is basically any positive $L^{q}$ function on $S^{\prime} \backslash S$. This is only possible if $\widehat{Q}_{S} \leq \widehat{Q}_{S^{\prime}}$ on $S^{\prime} \backslash S$, and as $\widehat{Q}_{S^{\prime}}=Q$ on $S^{\prime}$, we get $\widehat{Q}_{S} \leq Q$ on $S^{\prime} \backslash S$. Since $\widehat{Q}_{S} \leq Q$ holds automatically on $S$, we see that $\widehat{Q}_{S} \leq Q$ on $S^{\prime}$. The conclusion $S<S^{\prime}$ now follows from Proposition 6.4. The proof is complete.

The proof of Theorem 6.22 has the following consequence.

Corollary 6.23. Suppose $S^{\prime}$ is a local $Q$-droplet, and that $S \subset S^{\prime}$, where $S$ is compact and lacks $Q$-shallow points. If condition (ii) of Theorem 6.22 is fulfilled, then $S$ is a local $Q$-droplet, and $S<S^{\prime}$. 
Proof. As in the proof of Theorem 6.22, we get from condition (ii) of that theorem

$$
0 \leq \int_{S^{\prime} \backslash S} h \Delta Q \mathrm{~d} A=\int_{\mathbb{C}}\left[U^{Q, S}-U^{Q, S^{\prime}}\right] \Delta h \mathrm{~d} A=\int_{S}\left[U^{Q, S}-U^{Q, S^{\prime}}\right] \Delta h \mathrm{~d} A+\int_{S^{\prime} \backslash S}\left[U^{Q, S}-U^{Q, S^{\prime}}\right] \Delta h \mathrm{~d} A,
$$

provided that $h \in W^{2,1}(\mathbb{C})$ (local Sobolev class) is subharmonic in $\mathbb{C} \backslash S$ and harmonic in $\mathbb{C} \backslash S^{\prime}$, and bounded near infinity, with $h(\infty)=0$. As in the proof of Theorem 6.22, we choose $h$ as (minus) the logarithmic potential of $g$, where $g \in L^{q}\left(S^{\prime}\right)$ has $g \geq 0$ on $S^{\prime} \backslash S$ and

$$
\int_{S^{\prime}} g \mathrm{~d} A=0 \text {. }
$$

so that

$$
0 \leq \int_{S}\left[U^{Q, S}-U^{Q, S^{\prime}}\right] g \mathrm{~d} A+\int_{S^{\prime} \backslash S}\left[U^{Q, S}-U^{Q, S^{\prime}}\right] g \mathrm{~d} A,
$$

If we choose $g$ such that $g=0$ on $S^{\prime} \backslash S$, we have equality (since then the inequality applies to $-g$ as well):

$$
\int_{S}\left[U^{Q, S}-U^{Q, S^{\prime}}\right] g \mathrm{~d} A=0 .
$$

As $g$ is now arbitrary except that its integral over $S$ vanishes, we conclude that $U^{Q, S}-U^{Q, S^{\prime}}$ is constant on $S$. Call the constant $c: U^{Q, S}=c+U^{Q, S^{\prime}}$ on $S$. Since $S^{\prime}$ is a local $Q$-droplet, the function $U^{Q, S^{\prime}}+Q$ is constant on $S^{\prime}$ (cf. Corollary 5.17) and consequently, $U^{Q, S}$ is constant on $S$ (after all, $\left.S \subset S^{\prime}\right)$. We conclude that $S$ is a local $Q$-droplet. That $S<S^{\prime}$ now follows from Theorem 6.22.

Theorem 6.24. Let $\left\{S_{t}\right\}_{t \in I}$ be a containment chain of local $Q$-droplets. If, for almost every $t \in I$, the set int $S_{t}$ has finitely many components and $S_{t}=\operatorname{clos} \operatorname{int} S_{t}$, then $\left\{S_{t}\right\}_{t \in I}$ is a domination chain.

Proof. We consider the $W^{2, p}$-smooth function

$$
V(\xi, \eta ; t):=U^{Q, S_{t}}(\xi)-U^{Q, S_{t}}(\eta)=\int_{S_{t}} \log \left|\frac{z-\xi}{z-\eta}\right|^{2} \Delta Q(z) \mathrm{d} A(z), \quad t \in I,
$$

and note that

$$
V(\xi, \eta ; t)=\widehat{Q}_{S_{t}}(\eta)-\widehat{Q}_{S_{t}}(\xi), \quad t \in I .
$$

Let $\mu, v$ be two compactly supported Borel probability measures which are absolutely continuous with densities in $L^{q}$ for some $q, 1<q<+\infty$. We need the expression

$$
V^{\mu, v}(t):=\int_{C^{2}} V(\xi, \eta ; t) \mathrm{d} \mu(\xi) \mathrm{d} v(\eta)=\int_{S_{t}} U^{v-\mu} \Delta Q \mathrm{~d} A, \quad t \in I,
$$

where $U^{v-\mu}:=U^{v}-U^{\mu}$, and $U^{\mu}, U^{v}$ are the usual logarithmic potentials. The functions $U^{\mu}, U^{v}$ are in $W^{2, q}$ and therefore continuous (and bounded). The function $U^{v-\mu}$ is harmonic off $\operatorname{supp}(v-\mu)$, and its value at infinity is $U^{v-\mu}(\infty)=0$. We have

$$
V^{\mu, v}\left(t^{\prime}\right)-V^{\mu, v}(t)=\int_{S_{t^{\prime}} \backslash S_{t}} U^{v-\mu} \Delta Q \mathrm{~d} A, \quad \text { for } t, t^{\prime} \in I \text { with } t<t^{\prime},
$$

which gives

$$
\left|V^{\mu, v}\left(t^{\prime}\right)-V^{\mu, v}(t)\right| \leq\left\|U^{v-\mu}\right\|_{L^{\infty}(\mathbb{C})} \int_{S_{t^{\prime}} \backslash S_{t}} \Delta Q \mathrm{~d} A=\left(t^{\prime}-t\right)\left\|U^{v-\mu}\right\|_{L^{\infty}(\mathbb{C}),} \text { for } t, t^{\prime} \in I \text { with } t<t^{\prime},
$$

since $\Delta Q \geq 0$ a.e. on a local $Q$-droplet. It follows that the function $V^{\mu, v}$ is Lipschitz continuous, and therefore differentiable almost everywhere. In view of (6.8), its right derivative is

$$
\left[V^{\mu, v}\right]^{\prime}\left(t^{+}\right)=\lim _{t^{\prime} \rightarrow t^{+}} \frac{1}{t^{\prime}-t} \int_{S_{t^{\prime}} \backslash S_{t}} U^{v-\mu} \Delta Q \mathrm{~d} A=\int_{\partial S_{t}^{\infty}} U^{v-\mu} \mathrm{d} \omega_{\infty}^{(t)}
$$


by Proposition 6.20, with the possible exception of a countable set of $t^{\prime}$ s. If now supp $v \subset S_{t}$, the function $U^{v-\mu}$ becomes subharmonic (and bounded) in $\mathbb{C} \backslash S_{t}$, so by the maximum principle

$$
0=U^{v-\mu}(\infty) \leq \int_{\partial S_{t}^{\infty}} U^{v-\mu} \mathrm{d} \omega_{\infty}^{(t)} .
$$

We conclude that $\left[V^{\mu, v}\right]^{\prime}(t) \geq 0$ for a.e. $t$ with $\operatorname{supp} v \subset S_{t}$. Put

$$
t_{v}:=\inf \left\{t \in I: \operatorname{supp} v \subset S_{t}\right\},
$$

and note that for $t \in I$ with $t>t_{v}$ we have $\left[V^{\mu, v}\right]^{\prime}(t) \geq 0$ almost everywhere, and hence $V^{\mu, v}$ is increasing on that sub-interval:

$$
V^{\mu, v}(t) \leq V^{\mu, v}\left(t^{\prime}\right) \text { for } t, t^{\prime} \in I \text { with } t_{v}<t<t^{\prime} .
$$

Next, we let the probability measures $\mu, v$ get more and more concentrated, so that supp $\mu \rightarrow\{\xi\}$ and supp $v \rightarrow\{\eta\}$. The inequality (6.9) survives the limit process, and we obtain that

$$
V(\xi, \eta ; t) \leq V\left(\xi, \eta ; t^{\prime}\right) \text { for } t, t^{\prime} \in I \text { with } t_{\xi}<t<t^{\prime},
$$

where

$$
t_{\xi}:=\inf \left\{t \in I: \xi \in S_{t}\right\} .
$$

The short argument which justifies this involves choosing the support of $v$ cleverly, and this is made possible by the fact that a local $Q$-droplet lacks $Q$-shallow points. If we use (6.7), we see that (6.10) expresses that

$$
\widehat{Q}_{S_{t}}(\eta)-\widehat{Q}_{S_{t}}(\xi) \leq \widehat{Q}_{S_{t^{\prime}}}(\eta)-\widehat{Q}_{S_{t^{\prime}}}(\xi) \text { for } t, t^{\prime} \in I \text { with } t_{\xi}<t<t^{\prime} .
$$

Since for $t_{\xi}<t<t^{\prime}$ we have $\xi \in S_{t} \subset S_{t^{\prime}}$, we get that (cf. Proposition 4.5)

$$
\widehat{Q}_{S_{t}}(\xi)=\widehat{Q}_{S_{t^{\prime}}}(\xi)=Q(\xi)
$$

so that (6.11) simplifies:

$$
\widehat{Q}_{S_{t}}(\eta) \leq \widehat{Q}_{S_{t^{\prime}}}(\eta) \text { for } t, t^{\prime} \in I \text { with } t_{\xi}<t<t^{\prime} .
$$

By making clever choices of the point $\xi$ we can get $t_{\xi}$ to be as close to 0 as we need, and so

$$
\widehat{Q}_{S_{t}}(\eta) \leq \widehat{Q}_{S_{t^{\prime}}}(\eta) \text { for } t, t^{\prime} \in I \text { with } t<t^{\prime} .
$$

For $\eta \in S_{t^{\prime}}$ we have $\widehat{Q}_{S_{t^{\prime}}}(\eta)=Q(\eta)$, and we derive that for $t, t^{\prime} \in I$ with $t<t^{\prime}$, we have

$$
\widehat{Q}_{S_{t}}(\eta) \leq Q(\eta) \quad \eta \in S_{t^{\prime}} .
$$

By Proposition 6.4, we get $S_{t}<S_{t^{\prime}}$ for all $t, t^{\prime} \in I$ with $t<t^{\prime}$, and $\left\{S_{t}\right\}_{t \in I}$ is a domination chain.

\section{The Hele-Shaw equation}

7.1. Smooth curve families (laminations). We need the following definition.

Definition 7.1. A family of simple curves $\Gamma_{t}$ (where $t$ runs over some interval) in $\mathbb{C}$ is a $C^{\infty}$-smooth lamination if

(i) $\Gamma_{t} \cap \Gamma_{t^{\prime}}=\emptyset$ holds for $t \neq t^{\prime}$, and

(ii) Each curve has a local parametrization $z=\gamma_{t}(\theta)$ ( $\theta$ runs over some interval), such that the function $\gamma(\theta, t):=\gamma_{t}(\theta)$ is a local $C^{\infty}$-diffeomorphism.

We will alternatively use the term $C^{\infty}$-smooth curve family as synonymous to $C^{\infty}$-smooth lamination. We mention that it is of course also possible to define laminations with a lower degree of smoothness than $C^{\infty}$. The normal velocity $v_{n}=v_{n}(z), z \in \Gamma_{t}$, may be defined as follows:

$$
v_{n}:=\left\langle\partial_{t} \gamma, n\right\rangle=\frac{1}{\left|\partial_{\theta} \gamma\right|} \operatorname{Im}\left[\partial_{t} \gamma \partial_{\theta} \bar{\gamma}\right]
$$


where the inner product is that of $\mathbb{C} \cong \mathbb{R}^{2}$ and $n$ is a unit normal to $\Gamma_{t}$. It is easy to see that the definition does not depend on the choice of parametrization $\gamma$. Indeed, if we write

$$
\tilde{\gamma}_{t}(\vartheta)=\tilde{\gamma}(\vartheta, t):=\gamma(\theta(\vartheta, t), t),
$$

where $\vartheta \mapsto \theta(\vartheta, t)$ is a local diffeomorphism, then

$$
\partial_{t} \tilde{\gamma}=\partial_{\theta} \gamma \partial_{t} \theta+\partial_{t} \gamma, \quad \partial_{\vartheta} \tilde{\gamma}=\partial_{\theta} \gamma \partial_{\vartheta} \theta,
$$

so that

$$
\begin{aligned}
\frac{1}{\left|\partial_{\vartheta} \tilde{\gamma}\right|} \operatorname{Im}\left[\partial_{t} \tilde{\gamma} \partial_{\vartheta} \bar{\gamma}\right]=\frac{1}{\left|\partial_{\theta} \gamma \partial_{\vartheta} \theta\right|} \operatorname{Im}\left[\left|\partial_{\theta} \gamma\right|^{2} \partial_{t} \theta \partial_{\vartheta} \theta\right. & \left.+\partial_{t} \gamma \partial_{\theta} \bar{\gamma} \partial_{\vartheta} \theta\right] \\
& =\frac{\partial_{\vartheta} \theta}{\left|\partial_{\vartheta} \theta\right|} \frac{1}{\left|\partial_{\theta} \gamma\right|} \operatorname{Im}\left[\partial_{t} \gamma \partial_{\theta} \bar{\gamma}\right]= \pm \frac{1}{\left|\partial_{\theta} \gamma\right|} \operatorname{Im}\left[\partial_{t} \gamma \partial_{\theta} \bar{\gamma}\right]
\end{aligned}
$$

where there is a sign change if the coordinate change reverses the direction of the unit normal vector.

Lemma 7.2. Let $\Gamma_{t}$ be a $C^{\infty}$-smooth lamination of Jordan curves, such that the domain $D_{t}$ interior to $\Gamma_{t}$ increases with $t$. Then, for continuous $f: \mathbb{C} \rightarrow \mathbb{C}$, we have

$$
\frac{\mathrm{d}}{\mathrm{d} t} \int_{D_{t}} f \mathrm{~d} A=2 \int_{\Gamma_{t}} f v_{n} \mathrm{~d} s .
$$

Proof. We identify the area form with the area measure according to, e.g., $\mathrm{d} z \wedge \mathrm{d} \bar{z}=2 \pi \mathrm{id} A(z)$. We may assume that for $t, t_{0}$ close to one another with $t_{0}<t, D_{t} \backslash D_{t_{0}}$ is parametrized by $\gamma_{\tau}(\theta)=\gamma(\theta, \tau)$ where $0 \leq \theta \leq 1$ and $t_{0} \leq \tau<t$, with periodicity boundary conditions in $\theta: \gamma(0, \tau)=\gamma(1, \tau)$. We let $R\left(t_{0}, t\right)$ denote the rectangle $[0,1] \times\left[t_{0}, t\right]$, so that

$$
\int_{D_{t} \backslash D_{t_{0}}} f \mathrm{~d} A=\frac{1}{2 \pi \mathrm{i}} \int_{R\left(t_{0}, t\right)} f(\gamma(\theta, \tau)) \mathrm{d} \gamma \wedge \mathrm{d} \bar{\gamma}
$$

We calculate:

$$
\mathrm{d} \gamma \wedge d \bar{\gamma}=\left[\partial_{\theta} \gamma \partial_{t} \bar{\gamma}-\partial_{\theta} \bar{\gamma} \partial_{t} \gamma\right] \mathrm{d} \theta \wedge \mathrm{d} t=2 \mathrm{i} \operatorname{Im}\left[\partial_{\theta} \gamma \partial_{t} \bar{\gamma}\right] \mathrm{d} \theta \wedge \mathrm{d} t=2 \mathrm{i}\left|\partial_{\theta} \gamma\right| v_{n} \mathrm{~d} \theta \mathrm{d} t,
$$

where we have identified a form with the corresponding measure. We identify $\left|\partial_{\theta} \gamma\right| \mathrm{d} \theta$ as arc length along $\Gamma_{t}$, so that $\left|\partial_{\theta} \gamma\right| \mathrm{d} \theta=2 \pi \mathrm{d} s(\theta)$, and therefore,

$$
\frac{1}{2 \pi \mathrm{i}} \mathrm{d} \gamma \wedge \mathrm{d} \bar{\gamma}=2 v_{n} \mathrm{~d} s(\theta) \mathrm{d} t
$$

The assertion is now immediate.

7.2. The Hele-Shaw flow equation. We assume we have a $C^{\infty}$-smooth lamination of Jordan curves $\Gamma_{t}$, and let $D_{t}$ denote the interior domain while $\Omega_{t}$ is the exterior (unbounded) domain. We also write $K_{t}:=\operatorname{clos} D_{t}=\mathbb{C} \backslash \Omega_{t}$, so that $K_{t}$ is compact. The classical Hele-Shaw equation relates the normal velocity $v_{n}$ to the normal derivative of the Green function (for the Laplacian) of the exterior domain $\Omega_{t}$ when one of the two coordinates is the point at infinity (the factor $\frac{1}{4}$ comes from our choice of normalizations):

$$
v_{n}=\frac{1}{4} \partial_{n} G_{t} \text { on } \Gamma_{t} \text {, where } G_{t}=G\left(\cdot, \infty ; \Omega_{t}\right) .
$$

The Green function $G_{t}$ is always positive in $\Omega_{t}$ and vanishes along the boundary $\Gamma_{t}$, and $n$ is taken in the exterior direction, so that $\partial_{n} G_{t}>0$ on $\Gamma_{t}$. Actually, $\partial_{n} G_{t}$ is the Poisson kernel of $\Omega$ for the point at infinity, so that $\frac{1}{2} \partial_{n} G_{t}$ times normalized arc length measure has the interpretation of $\mathrm{d} \omega_{\infty}^{(t)}$, harmonic measure at infinity for the domain $\Omega_{t}$. There is also a weighted analog of (7.1): the weighted Hele-Shaw equation is

$$
\rho v_{n}=\frac{1}{4} \partial_{n} G_{t} \quad \text { on } \Gamma_{t}
$$


The function $\rho$ is the weight, and it is assumed to be $C^{\infty}$-smooth with $\rho>0$ point-wise. It is possible to interpret the introduction of the weight as a change of the geometry (cf. [16], [14], [15]). In the sequel, we will use $\rho=\Delta Q$.

Definition 7.3. We say that an increasing family of compact sets $\left\{K_{t}\right\}_{t}$ (where $t$ ranges over some interval) is a generalized solution of the weighted Hele-Shaw equation with weight $\rho=\Delta Q$ if

(i) $\Delta Q \geq 0$ on $\cup_{t} K_{t}$, if

(ii) for each $t \in I, K_{t}$ lacks $Q$-shallow points, and if,

(iii) for all $f \in C(\mathbb{C})$, the function

$$
t \mapsto \int_{K_{t}} f \Delta Q \mathrm{~d} A
$$

is absolutely continuous and for a.e. $t$ we have $\left(\Gamma_{t}=\partial \Omega_{t}^{\infty}\right.$ where $\Omega_{t}^{\infty}:=\mathbb{C} \backslash$ phull $\left(K_{t}\right)$ is the unbounded component of the complement $\mathbb{C} \backslash K_{t}$ and $\omega_{\infty}^{(t)}$ is harmonic measure at infinity for $\left.\Omega_{t}^{\infty}\right)$

$$
\frac{\mathrm{d}}{\mathrm{d} t} \int_{K_{t}} f \Delta Q \mathrm{~d} A=\int_{\Gamma_{t}} f \mathrm{~d} \omega_{\infty}^{(t)} .
$$

Note that no smoothness requirement is imposed on the compact sets $K_{t}$ as in the standard formulation of the weighted Hele-Shaw equation (7.2). The way things are set up, strong solutions of the weighted Hele-Shaw equation (i.e., solutions of (7.2) ) are automatically generalized solutions. In short, the equation asks that the compact sets $K_{t}$ grow according to the law

$$
\frac{\mathrm{d}}{\mathrm{d} t}\left[1_{K_{t}} \Delta Q \mathrm{~d} A\right]=\mathrm{d} \omega_{\infty}^{(t)} .
$$

Proposition 7.4. Let $\left\{K_{t}\right\}_{t}$ be an increasing family of compact sets, where $t$ ranges over an open interval $I$, and suppose $\Delta Q \geq 0$ on $\cup_{t \in I} K_{t}$, and that $K_{t}$ lacks $Q$-shallow points, for each $t \in I$. Then $\left\{K_{t}\right\}_{t \in I}$ is a generalized solution of the weighted Hele-Shaw equation with weight $\Delta Q$ if and only if, for all $t, t^{\prime} \in I$ with $t<t^{\prime}$, and for all real-valued $f \in C(\mathbb{C})$,

$$
\int_{K_{t^{\prime}} \backslash K_{t}} f \Delta Q \mathrm{~d} A=\int_{t}^{t^{\prime}} \int_{\Gamma_{\tau}} f \mathrm{~d} \omega_{\infty}^{(\tau)} \mathrm{d} \tau \text {. }
$$

Proof. This is just an application of Calculus.

So, the weighted Hele-Shaw equation corresponds to the disintegration of measures

$$
1_{K_{t^{\prime}} \backslash K_{t}} \Delta Q \mathrm{~d} A=\int_{t}^{t^{\prime}} \mathrm{d} \omega_{\infty}^{(\tau)} \mathrm{d} \tau
$$

It follows from the standard properties of the harmonic measure that if $f \in C(\mathbb{C})$ is real-valued, bounded, and subharmonic in $\mathbb{C} \backslash K_{t}$ while it is harmonic near infinity, then

$$
\int_{K_{t^{\prime}} \backslash K_{t}} f \Delta Q \mathrm{~d} A=\int_{t}^{t^{\prime}} \int_{\Gamma_{\tau}} f \mathrm{~d} \omega_{\infty}^{(\tau)} \mathrm{d} \tau \geq \int_{t}^{t^{\prime}} f(\infty) \mathrm{d} \tau=\left(t^{\prime}-t\right) f(\infty) .
$$

This strongly resembles Richardson's inequality for local $Q$-droplets (Theorem 6.22). The comparison with Theorem 6.22 suggests the concept of a weak solution to the Hele-Shaw equation.

Definition 7.5. We say that an increasing family of compact sets $\left\{K_{t}\right\}_{t}$ (where $t$ ranges over some interval) is a weak solution of the weighted Hele-Shaw equation with weight $\Delta Q$ if

(i) $\Delta Q \geq 0$ on $\cup_{t} K_{t}$, if

(ii) for each $t \in I, K_{t}$ lacks $Q$-shallow points, and if,

(iii) for all real-valued $f \in W^{2,1}(\mathbb{C})$ (local Sobolev class),

$$
\left(t^{\prime}-t\right) f(\infty) \leq \int_{K_{t^{\prime}} \backslash K_{t}} f \Delta Q \mathrm{~d} A \text { for } t, t^{\prime} \in I \text { with } t<t^{\prime},
$$

provided $f$ is subharmonic in $\mathbb{C} \backslash K_{t}$, harmonic in $\mathbb{C} \backslash K_{t^{\prime}}$, and bounded near infinity. 
Proposition 7.6. A generalized solution of the Hele-Shaw equation is a weak solution.

Proof. It is known that it suffices to have condition (iii) of Definition 7.5 fulfilled for $f \in W^{2, q}$ for some $q$ slightly bigger than 1 . Such functions are continuous, so the assertion is immediate from (7.3).

We note that the sets $K_{t}$ need not be local $Q$-droplets, although that is one particular instance. The analogy with that case suggest the following.

Definition 7.7. An increasing family of compact sets $\left\{K_{t}\right\}_{t}$ is correctly indexed if

$$
t=t\left(Q, K_{t}\right)=\int_{K_{t}} \Delta Q \mathrm{~d} A .
$$

This is in agreement with (7.3) (or with Definition 7.5) for $f \equiv 1$ (since the inequality applies to $f \equiv-1$ as well the inequality is of course an equality).

It is known that the Hele-Shaw equation behaves like the heat equation, in that one direction of time $t$ is stable and the other is unstable. Here, the evolution $t \mapsto K_{t}$ is unstable when $t$ increases, and stable when $t$ decreases.

Theorem 7.8. Let $K_{*} \subset \mathbb{C}$ be compact, with $\Delta Q \geq 0$ a.e. on $K_{*}$. We assume that $t_{*}:=t\left(Q, K_{*}\right)>0$, and that $K_{*}$ lacks $Q$-shallow points. Then there exists a correctly indexed weak solution $t \mapsto K_{t}$ of the weighted Hele-Shaw equation with weight $\Delta Q$ on the interval $0<t \leq t_{*}$, such that $K_{t_{*}}=K_{*}$. The solution is unique.

Proof. We consider the function $\widetilde{Q}_{*}:=-U^{Q, K_{*}}$, where $U^{Q, K_{*}}$ is as in (5.2). It has

$$
\Delta \widetilde{Q}_{*}=1_{K_{*}} \Delta Q, \quad \widetilde{Q}_{*}(z)=t_{*} \log |z|^{2}+\mathrm{O}(1) \text { as }|z| \rightarrow+\infty,
$$

and we can define

$$
K_{t}:=S_{t}\left[\widetilde{Q}_{*}, K_{*}\right], \quad 0<t<t_{*}
$$

and $K_{t_{*}}:=K_{*}$. The way things are set up, $K_{*}$ becomes a $\widetilde{Q}_{*}$-droplet (cf. Corollary 5.17). Moreover, in view of Lemma 6.1 (with $\widetilde{Q}_{*}$ in place of $Q$ ), the sets $K_{t}$ are local $\widetilde{Q}_{*}$-droplets. We see from Theorem 6.22 that the sets $K_{t}$ form a domination chain of local $\widetilde{Q}_{*}$-droplets if and only if they form a weak solution of the Hele-Shaw equation.

It remains to establish that the weak solution $t \mapsto K_{t}$ unique. So, suppose $t \mapsto K_{t}$ is a weak solution, which need not be of the form (7.4). We claim that $K_{t}$ is a $\widetilde{Q}_{*}$-droplet for $0<t<t_{*}$. We know that $K_{*}$ is a local $\widehat{Q}_{*}$-droplet, that $K_{t}$ has no $Q$-shallow points, and that $K_{t} \subset K_{*}$. In addition, the weak solution condition entails

$$
\left(t_{*}-t\right) f(\infty) \leq \int_{K_{*} \mid K_{t}} f \Delta Q \mathrm{~d} A, \quad 0<t<t_{*}
$$

provided $f \in W^{2,1}(\mathbb{C})$ (local Sobolev class) is real-valued, subharmonic in $\mathbb{C} \backslash K_{t}$, harmonic in $\mathbb{C} \backslash K_{*}$, and bounded near infinity. An application of Corollary 6.23 shows that $K_{t}$ must also be a local $\widetilde{Q}_{*}$-droplet, with $K_{t} \prec K_{*}$ with respect to the weight $\widetilde{Q}_{*}$. The uniqueness part is now a consequence of Lemma 6.11

Remark 7.9. (a) A key element of the proof of Theorem 7.8 is the identification of the weak solutions of the Hele-Shaw equation $t \mapsto K_{t}$ with domination chains with respect to the weight $\widetilde{Q}_{*}$.

(b) Theorem 7.8 supplies existence and uniqueness in the backward time direction. It is not difficult to see that there is even local uniqueness in the backward time direction. However, in the forward time direction, there is generally neither existence nor uniqueness. An example of non-uniqueness can be based on, e.g., the setting of Remark 6.7 We now discuss non-existence. In the context of Theorem 7.8 , the difference $U^{Q, K_{*}}-U^{Q, K_{t}}$ is constant on $K_{t}$ for $0<t<t_{*}$ (see, e.g., 
the proof of Corollary 6.23); let $c(t)$ be that constant. We consider the functions $H_{t}:=Q-U^{Q}, K_{t}$ and $H_{*}:=Q-U^{Q, K_{*}}$, which have $\Delta H_{t}=0$ a.e. on $K_{t}$ and $\Delta H_{*}=0$ a.e. on $K_{*}$, respectively. We have $H_{t}-H_{*}=U^{Q, K_{*}}-U^{Q, K_{t}}=c(t)$ on $K_{t}$, we we write as $H_{t}=c(t)+H_{*}$ on $K_{t}$. So $H_{t}$ restricted to $K_{t}$ is supposed to have an extension to $K_{*}$ - the function $\widetilde{H}_{t}:=c(t)+H_{*}-$ with $\Delta \widetilde{H}_{t}=0$ a.e. on $K_{*}$. This adds an additional smoothness requirement on $H_{t}$ for $0<t<t_{*}$, which suggests that $K_{t}$ cannot be an arbitrary compact subset of $\mathbb{C}$ with $\Delta Q \geq 0$ a.e. on $K_{t}$ which lacks $Q$-shallow points. But $K_{t}$ is uniquely given for $0<t<t_{*}$ (the backward direction) for arbitrary compacts $K_{*}$ lacking $Q$-shallow points. So with very irregular $K_{*}$ we should be able to arrange that we have non-existence in the forward time direction. Another reason for non-existence in the forward direction is the existence of maximal local $Q$-droplets (see the next section for details), at least for some $Q$ with $\Delta Q \equiv 1$.

A proof of the following statement can be based on Proposition 6.20. The only part that needs checking is the absolute continuity requirement, which we leave to the interested reader.

Proposition 7.10. Suppose $t \mapsto K_{t}$ is a weak solution to the Hele-Shaw equation and that for almost all $t$ the sets int $K_{t}$ have finitely many components. Then $t \mapsto K_{t}$ is a generalized solution.

\section{REFERENCES}

[1] Y. Ameur, H. Hedenmalm, N. Makarov, Berezin transform in polynomial Bergman spaces. Comm. Pure Appl. Math. 63 (2010), 1533-1584.

[2] Y. Ameur, H. Hedenmalm, N. Makarov, Fluctuations of eigenvalues of random normal matrices. Duke Math. J. 159 (2011), 31-81.

[3] Y. Ameur, H. Hedenmalm, N. Makarov, Random normal matrices and Ward identities. Submitted.

[4] R. J. Berman, Bergman kernels for weighted polynomials and weighted equilibrium measures of $\mathbb{C}^{n}$. Indiana Univ. Math. J. 58 (2009), no. 4, 1921-1946.

[5] L. A. Caffarelli, D. Kinderlehrer, Potential methods in variational inequalities. J. Anal. Math. 37 (1980), 285-295.

[6] J. L. Doob, Classical potential theory and its probabilistic counterpart. Reprint of the 1984 edition. Classics in Mathematics. Springer-Verlag, Berlin, 2001.

[7] N. Dunford, J. T. Schwartz, Linear operators. Part II. Spectral theory. Selfadjoint operators in Hilbert space. With the assistance of William G. Bade and Robert G. Bartle. Reprint of the 1963 original. Wiley Classics Library. A WileyInterscience Publication. John Wiley \& Sons, Inc., New York, 1988.

[8] P. Elbau, G. Felder, Density of eigenvalues of random normal matrices. Comm. Math. Phys. 259 (2005), no. 2, $433-450$.

[9] A. Friedman, Variational principles and free-boundary problems. Second edition. Robert E. Krieger Publishing Co., Inc., Malabar, FL, 1988.

[10] J. B. Garnett, D. E. Marshall, Harmonic measure. New Mathematical Monographs, 2. Cambridge University Press, Cambridge, 2005.

[11] I. C. Gohberg, M. G. Krein, Introduction to the theory of linear nonselfadjoint operators. Translated from the Russian by A. Feinstein. Translations of Mathematical Monographs, Vol. 18 American Mathematical Society, Providence, R.I. 1969.

[12] Gustafsson, B. Applications of variational inequalities to a moving boundary problem for Hele-Shaw flows. SIAM J. Math. Anal. 16 (1985), no. 2, 279-300.

[13] H. Hedenmalm, N. Makarov, Quantum Hele-Shaw flow. 2004 preprint. arXiv: math/0411437

[14] H. Hedenmalm, A. Olofsson, Hele-Shaw flow on weakly hyperbolic surfaces. Indiana Univ. Math. J. 54 (2005), no. 4, 1161-1180.

[15] H. Hedenmalm, Y. Perdomo G., Mean value surfaces with prescribed curvature form. J. Math. Pures Appl. (9) 83 (2004), no. 9, 1075-1107.

[16] H. Hedenmalm, S. Shimorin, Hele-Shaw flow on hyperbolic surfaces. J. Math. Pures Appl. (9) 81 (2002), no. 3, 187-222.

[17] K. Johansson, On fluctuations of eigenvalues of random Hermitian matrices. Duke Math. J. 91 (1998), no. 1, 151-204.

[18] D. Kinderlehrer, G. Stampacchia, An introduction to variational inequalities and their applications. Pure and Applied Mathematics, 88. Academic Press, 1980.

[19] Mehta, M. L., Random matrices. Third edition. Pure and Applied Mathematics (Amsterdam), 142. Elsevier/Academic Press, Amsterdam, 2004.

[20] E. B. Saff, V. Totik, Logarithmic potentials with external fields. Appendix B by Thomas Bloom. Grundlehren der Mathematischen Wissenschaften [Fundamental Principles of Mathematical Sciences], 316. Springer-Verlag, Berlin, 1997.

[21] P. B. Wiegmann, Aharonov-Bohm effect in the quantum Hall regime and Laplacian growth problems. Statistical field theories (Como, 2001), 337-349, NATO Sci. Ser. II Math. Phys. Chem., 73, Kluwer Acad. Publ., Dordrecht, 2002. 
Håkan Hedenmalm, Department of Mathematics, KTH Royal Institute of Technology, S - 10044 Stockholm, SWEDEN

E-mail address: haakanh@math.kth.se

Nikolai Makarov, Department of Mathematics, California Institute of Technology, Pasadena, CA 91125, USA

E-mail address: makarov@caltech.edu 